\title{
Two-loop superstring five-point amplitudes. Part II. Low energy expansion and S-duality
}

Eric D'Hoker, ${ }^{a}$ Carlos R. Mafra, ${ }^{b}$ Boris Pioline $^{c}$ and Oliver Schlotterer ${ }^{d}$

${ }^{a}$ Mani L. Bhaumik Institute for Theoretical Physics, Department of Physics and Astronomy, University of California, Los Angeles, CA 90095, U.S.A.

${ }^{b}$ STAG Research Centre and Mathematical Sciences, University of Southampton, Highfield, Southampton SO17 1BJ, U.K.

${ }^{c}$ Laboratoire de Physique Théorique et Hautes Energies, CNRS and Sorbonne Université, UMR 7589,

Campus Pierre et Marie Curie, 4 Place Jussieu 75252 Paris, France

${ }^{d}$ Department of Physics and Astronomy, Uppsala University, 75108 Uppsala, Sweden

E-mail: dhoker@physics.ucla.edu, c.r.mafra@soton.ac.uk, pioline@lpthe.jussieu.fr, oliver.schlotterer@physics.uu.se

Dedicated to the life, science, and art of Professor Jean-Loup Gervais 
ABSTRACT: In an earlier paper, we constructed the genus-two amplitudes for five external massless states in Type II and Heterotic string theory, and showed that the $\alpha^{\prime}$ expansion of the Type II amplitude reproduces the corresponding supergravity amplitude to leading order. In this paper, we analyze the effective interactions induced by Type IIB superstrings beyond supergravity, both for $\mathrm{U}(1)_{R}$-preserving amplitudes such as for five gravitons, and for $\mathrm{U}(1)_{R}$-violating amplitudes such as for one dilaton and four gravitons. At each order in $\alpha^{\prime}$, the coefficients of the effective interactions are given by integrals over moduli space of genus-two modular graph functions, generalizing those already encountered for four external massless states. To leading and sub-leading orders, the coefficients of the effective interactions $D^{2} \mathcal{R}^{5}$ and $D^{4} \mathcal{R}^{5}$ are found to match those of $D^{4} \mathcal{R}^{4}$ and $D^{6} \mathcal{R}^{4}$, respectively, as required by non-linear supersymmetry. To the next order, a $D^{6} \mathcal{R}^{5}$ effective interaction arises, which is independent of the supersymmetric completion of $D^{8} \mathcal{R}^{4}$, and already arose at genus one. A novel identity on genus-two modular graph functions, which we prove, ensures that up to order $D^{6} \mathcal{R}^{5}$, the five-point amplitudes require only a single new modular graph function in addition to those needed for the four-point amplitude. We check that the supergravity limit of $\mathrm{U}(1)_{R}$-violating amplitudes is free of $\mathrm{UV}$ divergences to this order, consistently with the known structure of divergences in Type IIB supergravity. Our results give strong consistency tests on the full five-point amplitude, and pave the way for understanding S-duality beyond the BPS-protected sector.

KeYwords: String Duality, Superstrings and Heterotic Strings, Conformal Field Models in String Theory

ARXIV EPRINT: 2008.08687 


\section{Contents}

1 Introduction 1

2 Review of the four- and five-point amplitudes $\quad 6$

$\begin{array}{lll}2.1 & \text { Chiral splitting } & 7\end{array}$

2.2 The chiral correlator 8

$\begin{array}{ll}2.3 \text { Scalar and vector superspace building blocks } & 10\end{array}$

$\begin{array}{lll}2.3 .1 & \text { Local building blocks } & 10\end{array}$

$\begin{array}{ll}\text { 2.3.2 Non-local building blocks } & 11\end{array}$

$\begin{array}{lll}2.4 & \text { Effective rules for bosonic components } & 12\end{array}$

2.4.1 Symmetries and relations of the effective components 13

$\begin{array}{lll}2.4 .2 & \text { Effective BRST invariants and correlators } & 14\end{array}$

$\begin{array}{lll}2.5 & \text { Assembling and expanding } & 15\end{array}$

3 The $\alpha^{\prime}$ expansion of genus-two integrals $\quad 17$

$\begin{array}{lll}3.1 & \text { Genus-two integrals occurring in Type II amplitudes } & 17\end{array}$

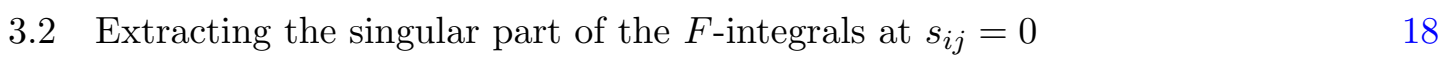

$\begin{array}{lll}3.3 & \text { Genus-two modular graph functions up to order } D^{6} \mathcal{R}^{5} & 19\end{array}$

3.4 Novel modular graph function identities 21

3.5 Expansion in $\alpha^{\prime}$ of the basic genus-two integrals 22

3.6 Decomposing the five-point correlator 23

4 The $\alpha^{\prime}$ expansion of genus-two amplitudes $\quad 25$

$\begin{array}{lll}4.1 & \text { The four-point amplitude } & 25\end{array}$

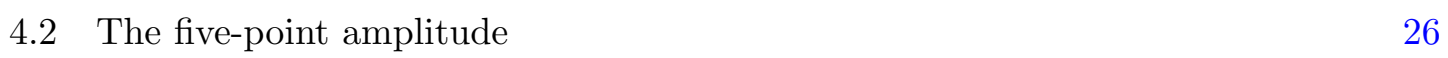

$\begin{array}{lll}\text { 4.2.1 Terms of order } D^{2} \mathcal{R}^{5} & 27\end{array}$

4.2.2 Terms of order $D^{4} \mathcal{R}^{5} \quad 28$

$\begin{array}{ll}\text { 4.2.3 Terms of order } D^{6} \mathcal{R}^{5} & 29\end{array}$

4.3 Components in Type IIB 30

4.3.1 Five-point tree-level amplitudes of SYM 31

4.4 Components in Type IIA 32

4.4.1 Five gravitons in Type IIA 33

4.4.2 Four gravitons and one $B$-field in Type IIA 34

4.5 Type IIB 5-point amplitudes up to genus-two 34

$\begin{array}{lll}\text { 4.5.1 Tree level } & 35\end{array}$

$\begin{array}{lll}\text { 4.5.2 Genus one } & 35\end{array}$

4.5.3 Genus two 36 
5 Consistency with supergravity and S-duality

5.1 R-symmetry violation and UV divergences in supergravity 37

$\begin{array}{lll}\text { 5.1.1 D-dimensional dilatons at genus two } & 38\end{array}$

$\begin{array}{lll}\text { 5.1.2 } D \text {-dimensional dilatons at genus one } & 39\end{array}$

$\begin{array}{lll}5.2 & \text { S-duality analysis } & 40\end{array}$

A Functions on Riemann surfaces $\quad 42$

A.1 Convention for forms $\quad 42$

A.2 Holomorphic 1-forms and the period matrix 42

A.3 The bi-holomorphic forms $\Delta$ and $\nu \quad 43$

A.4 Some useful integrals 44

A.5 The Arakelov Green function 44

A.6 Reducing integrals of Arakelov Green functions 45

$\begin{array}{ll}\text { B Expanding the integrals } & 45\end{array}$

B.1 The $H$-integrals 46

B.2 The $G$-integrals $\quad 46$

B.3 The $J$-integrals 46

$\begin{array}{lll}\text { B.3.1 First order in } s & 47\end{array}$

$\begin{array}{lll}\text { B.3.2 Second order in } s & 48\end{array}$

B.4 The $F$-integrals $\quad 51$

C Degenerations of genus-two modular graph functions $\quad 52$

$\begin{array}{ll}\text { C.1 The non-separating degeneration } & 52\end{array}$

$\begin{array}{ll}\text { C.1.1 Useful integrals } & 54\end{array}$

C.1.2 Non-separating degeneration of $\varphi$ and $\mathcal{Z}_{1,2,3} \quad 55$

$\begin{array}{lll}\text { C.1.3 Non-separating degeneration of } \mathcal{Z}_{4} & 55\end{array}$

$\begin{array}{lll}\text { C.1.4 Non-separating degeneration of } \mathcal{Z}_{5} & 58\end{array}$

C.1.5 A novel identity for genus-one elliptic modular graph functions $\quad 60$

$\begin{array}{lll}\text { C.2 Separating degeneration } & 61\end{array}$

$\begin{array}{lll}\text { C.3 Tropical limit } & 62\end{array}$

$\begin{array}{ll}\text { D Proof of the modular graph function identities } & 64\end{array}$

E Overall normalization of the genus-two amplitude $\quad 66$

\section{Introduction}

Scattering amplitudes of massless states are the basic observables in string theory and, in principle, are well-defined at arbitrary order in perturbation theory (for reviews see [1-4]). They are UV-finite by construction and, in the $\alpha^{\prime}$ expansion, reduce to supergravity amplitudes plus an infinite series in $\alpha^{\prime}$ of effective interactions [5]. In practice, however, the 
explicit evaluation of superstring amplitudes rapidly becomes prohibitively complicated beyond genus one. For a long time the state of the art has been the four-point genus-two amplitude which was constructed in the Ramond-Neveu-Schwarz (RNS) formalism (see [6] and references therein), reproduced in the pure spinor (PS) formalism and extended to include external fermions [7].

Beyond this, partial results have been obtained in the PS formalism for the five-point two-loop amplitude [8], and the four-point three-loop amplitude [9]. A major obstacle to explicit evaluations in the PS formalism (in its non-minimal version) is due to the composite $b$-ghost [10], which diverges at the origin of the cone of pure spinor zero-modes and requires a large number of Wick contractions. As a consequence, in both cases the string integrand was determined only up to regular terms (multiplied by the usual Koba-Nielsen factor). These ambiguities do not affect the leading behavior as $\alpha^{\prime} \rightarrow 0$, which was successfully matched to the UV divergence of the respective supergravity integrands.

Recently, by combining the non-minimal pure spinor formalism with the chiral splitting formalism initially developed for the RNS formalism [1, 11], we obtained the full genus-two amplitude for five arbitrary massless external states in Type II and Heterotic strings [12]. This result followed from two key requirements imposed on the amplitude, namely BRST invariance along with invariance under "homology shifts", which consist of the combined action of taking one vertex point around a homology cycle on the genus-two surface, and shifting the corresponding loop momentum. It turns out that these requirements are strong enough to fix the chiral amplitude completely, given the operator product expansion (OPE) singularities between the canonical worldsheet fields. The full amplitude is obtained by assembling the chiral amplitudes for the left- and right-movers (or the chiral amplitude with the Chan-Paton factors for open strings), and integrating over loop momenta, vertex points, and moduli of the genus-two surface.

To leading order in the $\alpha^{\prime} \rightarrow 0$ expansion, the integral giving the string amplitude was shown to reproduce the kinematic numerators of the two-loop five-point supergravity diagrams, which were computed for four-dimensional $\mathcal{N}=8$ supergravity in [13] and for ten-dimensional Type II supergravity states in [14]. In a companion paper [15], the genus-two amplitude for five NS states will be derived from first principles within the RNS formalism.

In this paper, we shall use the results of [12] as the starting point for a systematic analysis of the low energy expansion of the five-point amplitude beyond leading order. Such an analysis is part of a general endeavor to understand the structure of the low energy effective action in superstring theories both in perturbation theory and at the non-perturbative level. For Type IIB superstring theory in 10-dimensional Minkowski space-time, S-duality allows one to make sharp and quantitative predictions of non-perturbative contributions to certain protected couplings. Specifically, combining perturbative results at tree-level and genus-one orders for the four-graviton scattering amplitude with requirements of spacetime supersymmetry and S-duality invariance [16-20], the axion-dilaton dependence of the coefficients of the effective interactions of the form $\mathcal{R}^{4}, D^{4} \mathcal{R}^{4}$ and $D^{6} \mathcal{R}^{4}$ were determined in terms of non-holomorphic modular functions of $\mathrm{SL}(2, \mathbb{Z})$. This has been accomplished not only in ten dimensions but also after compactification on a torus, in terms of certain automorphic functions of the U-duality group (see e.g. [21-23] and references therein). 
The analytic structure of the genus-one four-graviton amplitude was established in [24] based on the moduli-space integrand in [5]. Perturbative contributions to the effective interactions $\mathcal{R}^{4}, D^{4} \mathcal{R}^{4}$ and $D^{6} \mathcal{R}^{4}$ were extracted and analyzed at genus one in [25-27], and at genus two in [28-30], the analysis being extended up to order $D^{8} \mathcal{R}^{4}$ in [31, 32]. The integrand at a given order is a linear combination of "modular graph functions" (MGFs), a class of real analytic modular functions which arise by integrating products of Green functions over the vertex points [31,33]. However, while these perturbative contributions are under analytic control, supersymmetry and S-duality no longer appear to determine the full automorphic forms under the S-duality group beyond $D^{6} \mathcal{R}^{4}$.

For five-graviton scattering, the low energy expansion has so far been considered systematically at tree level [34] and one loop [35, 36] only, while a preliminary analysis of the two-loop amplitude at leading order was performed in [8]. A key result from the one-loop analysis in [35] was that the five-point integrand at any order in $\alpha^{\prime}$ can be expressed as a linear combination of MGFs similar to the four-point case. Moreover, the very same linear combinations were found to govern the five-point $D^{2 k} \mathcal{R}^{5}$ and four-point $D^{2 k+2} \mathcal{R}^{4}$ interactions for $k=1,2$. Since the tree-level coefficients are also identical - namely $\zeta_{5}$ in case of $D^{4} \mathcal{R}^{4}, D^{2} \mathcal{R}^{5}$ and $\zeta_{3}^{2}$ in case of $D^{6} \mathcal{R}^{4}, D^{4} \mathcal{R}^{5}$ - this suggests that both interactions are related by non-linear supersymmetry and are multiplied by the same automorphic form.

For the $D^{2 k} \mathcal{R}^{5}$ and $D^{2 k+2} \mathcal{R}^{4}$ effective interactions at $k \geq 3$, by contrast, it was found [35] that new linear combinations of MGFs occur in the five-point amplitude, which indicates the presence of new supersymmetric invariants not present at tree level. The first example of this occurs for $k=3$, leading to a five-point effective interaction which we denote by $\left(D^{6} \mathcal{R}^{5}\right)^{\prime}$ to distinguish it from the $D^{6} \mathcal{R}^{5}$ interaction related by non-linear supersymmetry to $D^{8} \mathcal{R}^{4}$.

Another key aspect of the one-loop analysis in [35] was the study of amplitudes violating the $\mathrm{U}(1)_{R}$ global symmetry of classical ten-dimensional Type IIB supergravity: due to a one-loop anomaly [37], $n$-point string amplitudes may violate the conservation of $\mathrm{U}(1)_{R}$ charge by up to $\pm 2(n-4)$ units (see e.g. [38-40]). At five points, this violation occurs for 1-dilaton 4-graviton scattering, schematically denoted by $\phi \mathcal{R}^{4}$, or 3-gravitons 2-KalbRamond fields, ${ }^{1}$ denoted by $G^{2} \mathcal{R}^{3}$, which are both maximally R-violating amplitudes in the language of [39]. In this case the automorphic form multiplying these interactions can no longer be invariant under S-duality, but must carry a modular weight so as to cancel the phase variation of the interaction vertex under S-duality. At low orders in $\alpha^{\prime}$, the analysis of [35] indicates that the automorphic form for $\mathrm{U}(1)_{R^{-v}}$-violating interactions is related to the automorphic function for the $\mathrm{U}(1)_{R}$-preserving ones by a raising operator (or modular derivative), which suggests that both interactions are part of the same supersymmetric invariant. However, this correspondence breaks down for $k=5$, where a $\mathrm{U}(1)_{R^{-v}}$ violating interaction of the form $D^{12} G^{2} \mathcal{R}^{3}$ arises which is not related to any $\mathrm{U}(1)_{R^{-}}$-preserving interaction of type $D^{10} \mathcal{R}^{5}$.

\footnotetext{
${ }^{1}$ By a slight abuse of nomenclature, we refer to the complex combination of RR and NS two-form fields in Type IIB supergravity as the Kalb-Ramond field, and denote its 3 -form field strength by $G$. In our conventions the dilaton fluctuation $\phi$ carries 2 units of $\mathrm{U}(1)_{R}$-charge, $G$ carries one unit and $\mathcal{R}$ is neutral.
} 
In this paper, we analyze the first few orders in the low energy expansion of the genustwo 5-point amplitude of [12], for various choices of external massless states of Type IIB and IIA superstrings. In general, we find that, at each order, the integrand on genus-two moduli space is a linear combination of genus-two MGFs, a class of real-analytic Siegel modular functions which arise by integrating products of Arakelov Green functions (and partial derivatives thereof) against suitable top forms on multiple copies of the genus-two curve $\Sigma[31,32]$. Quite remarkably, we find that the many MGFs occurring at order $D^{6} \mathcal{R}^{5}$ (some of which previously considered in [41]) can all be reduced to linear combinations of 5 basic ones $\mathcal{Z}_{1}, \ldots, \mathcal{Z}_{5}$ defined in (3.14) below, along with the square $\varphi^{2}$ of the KawazumiZhang invariant $\varphi$; the latter occurs in the four-point amplitude at order $D^{6} \mathcal{R}^{4}$ [29], and reappears in the five-point amplitude at order $D^{4} \mathcal{R}^{5}$. The graphs for the relevant genus-two MGFs are presented in figure 1.

Moreover, we find that one of these six MGFs can be eliminated by virtue of a novel identity amongst five of them,

$$
\mathcal{Z}_{1}+\mathcal{Z}_{2}+\mathcal{Z}_{3}+\frac{1}{2} \mathcal{Z}_{4}-\varphi^{2}=0
$$

This identity is quite remarkable since it relates different graph topologies, and can be viewed as a genus-two analogue of the identities between genus-one MGFs proven in [42-45]. It would be interesting to revisit the analysis of the Laplace equation on genus-two modular graph functions in [41] in view of the identity (1.1) and the simpler identities (A.16).

In the non-separating degeneration limit, identity (1.1) implies a novel identity (3.16) for genus-one elliptic MGFs, ${ }^{2}$ which suggests that the identites of [42-45] may admit far reaching generalizations in the elliptic and Siegel cases. The identity (1.1) is motivated by the analysis of degeneration limits in appendix $\mathrm{C}$, and derived in appendix D by exploiting a novel lemma (D.1), which relates derivatives $\partial_{z_{i}} \mathcal{G}\left(z_{i}, z_{j}\right)$ and $\partial_{z_{j}} \mathcal{G}\left(z_{i}, z_{j}\right)$ of the Arakelov Green function at arbitrary genus. Another interesting fact is that the MGF $\mathcal{Z}_{5}$ involving two derivatives of Green functions tends to zero both in the separating and non-separating degenerations, unlike the others which diverge in both limits, so that it leaves no trace in the supergravity limit.

The details of the string integrand on moduli space depend on the order in the expansion and the choice of external massless states of the Type IIB multiplet, as follows.

- In the $\mathrm{U}(1)_{R}$-preserving sector, at order $D^{2 k} \mathcal{R}^{5}$ with $k=1,2$, we find the same integrand (namely the constant measure $d \mu_{2}$ on the Siegel upper half plane at order $D^{2} \mathcal{R}^{5}$, and the Kawazumi-Zhang measure $\varphi d \mu_{2}$ at order $D^{4} \mathcal{R}^{5}$ ) as for the four-point amplitude at order $D^{2 k+2} \mathcal{R}^{4}$, up to overall normalization. This supports the expectation that the $D^{2} \mathcal{R}^{5}$ and $D^{4} \mathcal{R}^{5}$ interactions belong to the same non-linear supersymmetric invariant as the $D^{4} \mathcal{R}^{4}$ and $D^{6} \mathcal{R}^{4}$ interactions, respectively, and should appear with the same automorphic coefficient in the low energy effective action, denoted by $\mathcal{E}_{(1,0)}$ and $\mathcal{E}_{(0,1)}$ in the standard fashion after [25].

\footnotetext{
${ }^{2}$ Elliptic MGFs are real-analytic functions of $(\tau, v)$, which are doubly periodic in $v$ and modular invariant; they can be obtained from the conventional MGFs of [33] by leaving one vertex position unintegrated, and have also been referred to as generalized MGFs in [32].
} 
- In the $\mathrm{U}(1)_{R}$-preserving sector, at order $D^{6} \mathcal{R}^{5}$, we find two distinct kinematic structures, one identical to the tree-level interaction, and the other identical to the genusone $\left(D^{6} \mathcal{R}^{5}\right)^{\prime}$ effective interaction. In the former case, the integrand is proportional to the same combination $\mathcal{Z}_{1}-2 \mathcal{Z}_{2}+\mathcal{Z}_{3}$ of genus-two MGFs appearing at order $D^{8} \mathcal{R}^{4}$ in four-graviton scattering, with the correct coefficient relative to the tree-level and genus-one amplitude. This confirms that $D^{8} \mathcal{R}^{4}$ and $D^{6} \mathcal{R}^{5}$ belong to a single supersymmetric invariant, with an automorphic coefficient $\mathcal{E}_{(2,0)}$ receiving tree-level up to genus-two contributions (and presumably higher genera as well). By contrast, the integrand for the genus-two $\left(D^{6} \mathcal{R}^{5}\right)^{\prime}$ involves the new MGFs $\mathcal{Z}_{4}, \mathcal{Z}_{5}$ and $\varphi^{2}$ (one of which can be eliminated by virtue of (1.1)). Along with the genus-one amplitude computed in [35], this predicts the first two terms in the weak coupling expansion of a new automorphic coefficient $\mathcal{E}_{(2,0)^{\prime}}$ which presumably also involves contributions of arbitrary genera.

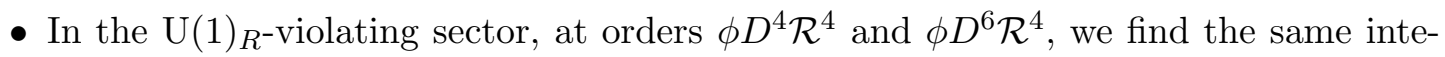
grand as in the $\mathrm{U}(1)_{R}$-preserving sector, up to a relative coefficient $-3 / 5$ and $-1 / 3$, respectively. As we explain in section 5 , this is consistent with linear supersymmetry and S-duality, which relate the ratio of coefficients of the $D^{2 k} \mathcal{R}^{5}$ and $\phi D^{2 k+2} \mathcal{R}^{4}$ at different loop orders by the action of a raising operator (or modular covariant derivative operator). At the next order, there are again two different kinematic structures $\phi D^{8} \mathcal{R}^{4}$ and $\left(\phi D^{8} \mathcal{R}^{4}\right)^{\prime}$, as in the one-loop 5-point amplitude [35]. For the first, the integrand is equal to the one for $D^{6} \mathcal{R}^{5}$ up to a relative coefficient $1 / 7$, consistent with linear supersymmetry. For the second, there is no obvious relation between the $\left(D^{6} \mathcal{R}^{5}\right)^{\prime}$ and $\left(\phi D^{8} \mathcal{R}^{4}\right)^{\prime}$ integrands, except for the fact that they are both linear combinations of the same MGFs $\mathcal{Z}_{i}, \varphi^{2}$ (subject to the relation (1.1)). By requiring that the integrated couplings be related by linear supersymmetry, we predict a relation between the divergent parts of the modular integrals on $\mathcal{M}_{2}$, which we check against the behavior of the integrand in the non-separating degeneration limit.

- Extracting the supergravity limit of the 1-dilaton, 4-graviton amplitude in any dimension $D$, we confirm the absence of UV divergences in this sector, in agreement with the known structure of UV divergences in supergravity at two loops [46]. The consistency of the low energy expansion with supersymmetry and S-duality provides a strong check on the full five-point amplitude constructed in [12].

Before proceeding further, we make two important comments. First, the notation $D^{2 k} \mathcal{R}^{5}$ is a moniker for the Taylor coefficient of order $p^{2 k+10}$ in the momentum expansion of the 5 -graviton amplitude; in general it includes both irreducible contributions from local interactions of the form $D^{2 k} \mathcal{R}^{5}$ in the low energy effective action, where $\mathcal{R}$ is the Riemann tensor and $D$ are covariant derivatives, with indices suitably contracted with the metric tensor, as well as reducible contributions from local interactions of the form $D^{2 k+2} \mathcal{R}^{4}$ and supergravity vertices. We do not attempt to disentangle these various contributions at two loops, but rather express the kinematic dependence of the Taylor coefficients at two loops in terms of tensorial quantities appearing at tree level or one loop; the procedure 
for subtracting reducible diagrams is then identical to the one required at these lower orders (see e.g. [47] at genus one). The same holds for the notation $\phi D^{2 k+2} \mathcal{R}^{4}$, which is a moniker for the Taylor coefficient of order $p^{2 k+10}$ in the momentum expansion of the 1-dilaton 4-graviton amplitude. Note that the constraints of S-duality on the low energy effective action translate directly into constrains on the corresponding Taylor coefficients in the amplitudes [35].

The second comment is that in certain space-time dimensions $D$ correlated with the order in the $\alpha^{\prime}$ expansion, these local effective interactions can mix with non-local interactions mediated by massless particles. In such cases a sliding scale must be specified to separate these effects $[48,49]$. This is in particular the case for the $D^{8} \mathcal{R}^{4}$ and $D^{6} \mathcal{R}^{5}$ interactions in $D=10$. Since we are mostly interested in the integrand, we shall mostly ignore these issues in this paper, except at some places in sections 4 and 5 .

Organization. The remainder of this paper is organized as follows. In section 2 we review the necessary results from paper [12] on the structure of the genus-two amplitude for five external massless states, and give simplified effective rules to extract the contribution from bosonic external states. In section 3 we decompose the genus-two five-point amplitude into a sum of products of kinematic factors times integrals in the vertex points on the genus-two Riemann surface, perform the $\alpha^{\prime}$ expansion of these integrals up to orders high enough to access the effective interactions of order $D^{6} \mathcal{R}^{5}$, and prove the above-mentioned identity between genus-two MGFs. In section 4, we extract the actual effective interactions up to order $D^{6} \mathcal{R}^{4}$, and present simplified concrete formulas for the separate cases of Type IIA and Type IIB superstrings. In section 5 we compare our perturbative results with predictions from S-duality and from the structure of UV divergences in supergravity. An overview of the function theory on Riemann surfaces of genus two is presented in appendix A; the detailed calculations of the $\alpha^{6}$ expansion of the genus-two integrals is given in section $\mathrm{B}$; the analysis of the non-separating, separating, and tropical degenerations of the integrals is given in appendix C; the identity (1.1) is proved in appendix D and details on the overall normalization of the genus-two amplitude are given in appendix E.

\section{Review of the four- and five-point amplitudes}

In this section, we review the structure of the genus-two chiral superstring amplitude for five massless states, as well as the physical amplitude in Type II string theory obtained by pairing left and right chiral amplitudes constructed in [12]. For comparison we also include the genus-two amplitude for four massless NS states, first computed in the RNS formalism in $[6,28,50]$ (based on the genus-two measure constructed in [51-54] which was re-derived using methods of algebraic geometry in [55]), and reproduced in the PS formalism and extended to include external fermions in $[7,56,57]$. Finally, we shall present a set of effective rules to extract the massless Neveu-Schwarz content of the pure spinor building blocks. These rules will allow us to re-express the results of [12], and of section 4 of this paper, in terms of the familiar $t_{8}$ and $\epsilon_{10}$ tensors and thereby facilitate the comparison with the RNS genus-two computation in [15]. 


\subsection{Chiral splitting}

The construction of the full integrand in [12] hinges on chiral splitting [1, 11], which allows us to decompose the integrand of the amplitude at fixed loop momentum into the product of chiral and anti-chiral amplitudes, associated to the left- and right-movers, respectively, ${ }^{3}$

$$
\mathcal{A}_{(N)}^{\text {genus }-2}=\delta(k) \mathcal{N}_{(N)} \int_{\mathcal{M}_{2}}\left|d^{3} \Omega\right|^{2} \int_{\Sigma^{N}} \int_{\mathbb{R}^{20}} d p \mathcal{F}_{(N)}\left(z_{i}, k_{i}, p^{I}\right) \overline{\tilde{\mathcal{F}}_{(N)}\left(z_{i},-\bar{k}_{i},-p^{I}\right)}
$$

Here, $\mathcal{M}_{2}$ is a fundamental domain in the rank 2 Siegel upper-half space, which may be parametrized locally by the period matrix $\Omega_{I J}$ and $d^{3} \Omega=d \Omega_{11} d \Omega_{12} d \Omega_{22}$ is the holomorphic top form on $\mathcal{M}_{2}$. The loop momenta for genus two are $p^{I}=\left(p^{1}, p^{2}\right)$ with $p^{1}, p^{2} \in \mathbb{R}^{10}$ and the volume form for the integration over loop momenta is $d p=d^{10} p^{1} d^{10} p^{2}$. The chiral and anti-chiral amplitudes may be further decomposed as follows,

$$
\mathcal{F}_{(N)}=\left\langle\mathcal{K}_{(N)}\right\rangle_{0} \mathcal{I}_{(N)}, \quad \tilde{\mathcal{F}}_{(N)}=\left\langle\tilde{\mathcal{K}}_{(N)}\right\rangle_{0} \mathcal{I}_{(N)}
$$

where $\left\langle\mathcal{K}_{(N)}\right\rangle_{0}$ and $\left\langle\tilde{\mathcal{K}}_{(N)}\right\rangle_{0}$ are the left- and right-moving chiral correlators, which will be discussed in detail in subsection 2.2, and $\mathcal{I}_{(N)}$ is the chiral Koba-Nielsen factor. Finally, the prefactor $\mathcal{N}_{(N)}$ is a normalization factor, which will include the dependence on the dilaton vacuum expectation value, and which we shall fix in section 4 .

The chiral Koba-Nielsen factor depends on the positions of the vertex operators $z_{i}$, the external momenta $k_{i}$ and the loop momenta $p^{I}$ and is given by the following universal formula, independently of the particular string theory under consideration, ${ }^{4}$

$$
\mathcal{I}_{(N)}\left(z_{i}, k_{i}, p^{I}\right)=\exp \left\{i \pi \Omega_{I J} p^{I} \cdot p^{J}+\sum_{i=1}^{N} 2 \pi i p^{I} \cdot k_{i} \int_{z_{0}}^{z_{i}} \omega_{I}-\sum_{i<j}^{N} s_{i j} \ln E\left(z_{i}, z_{j}\right)\right\}
$$

where $\omega_{I}$ are holomorphic Abelian differentials, $\Omega_{I J}$ are the components of the period matrix, and $E$ is the prime form. The dimensionless kinematic variables $s_{i j}$ are defined by,

$$
s_{i j}=-\frac{\alpha^{\prime}}{2} k_{i} \cdot k_{j}
$$

The chiral Koba-Nielsen factor $\mathcal{I}_{(N)}$, as well as the full chiral amplitude $\mathcal{F}_{(N)}$, enjoy two fundamental properties $[1,11]$ : they are locally holomorphic in $z_{i}$ and $\Omega_{I J}$ and are invariant under combined shifts of the points $z_{i}$ by homology cycles $\mathfrak{A}_{J}, \mathfrak{B}_{J}$, multiplication by a phase, and a shift in loop momenta, given as follows for $\mathcal{I}_{(N)}$,

$$
\begin{aligned}
& \mathcal{I}_{(N)}\left(z_{i}, k_{i}, p^{I}\right)=e^{-2 \pi i p_{J} \cdot k_{j}} \mathcal{I}_{(N)}\left(z_{i}+\delta_{i j} \mathfrak{A}_{J}, k_{i}, p^{I}\right) \\
& \mathcal{I}_{(N)}\left(z_{i}, k_{i}, p^{I}\right)=\mathcal{I}_{(N)}\left(z_{i}+\delta_{i j} \mathfrak{B}_{J}, k_{i}, p^{I}-\delta_{J}^{I} k_{j}\right)
\end{aligned}
$$

We refer to these combined transformations as homology shifts. The complex conjugate of the anti-chiral amplitude $\tilde{\mathcal{F}}_{(N)}$ satisfies the above homology shift invariance with inverse phase factor. As a result, the integral over loop momenta of the product of chiral and antichiral amplitudes is single-valued in each $z_{i}$ and produces a well-defined integral over $\Sigma^{N}$.

\footnotetext{
${ }^{3}$ Throughout we denote $\delta(k)=(2 \pi)^{10} \delta^{(10)}\left(\sum_{i} k_{i}\right)$ where $k_{i}$ are the momenta of the external states.

${ }^{4}$ Our conventions will follow those of appendix B in [12] and are summarized in appendix A of this paper. In particular, we adopt the Einstein summation conventions for repeated indices $I, J, \ldots=1,2$ and often abbreviate the point $z_{i}$, as an argument of a function, simply by $i$, for example in $\Delta(i, j)=\Delta\left(z_{i}, z_{j}\right)$ below.
} 


\subsection{The chiral correlator}

The chiral correlator $\left\langle\mathcal{K}_{(N)}\right\rangle_{0}$ depends on the same data as $\mathcal{I}_{(N)}$, along with the leftmoving polarization vectors $\varepsilon_{i}^{m}$ and spinors $\chi_{i}^{\alpha}$ describing the external states of the tendimensional super-Yang-Mills (SYM) multiplet. The function $\mathcal{K}_{(N)}$ further depends on the zero modes of the spinor fields $\theta^{\alpha}, \lambda^{\alpha}$ (subject to the pure spinor constraint $\lambda \gamma^{m} \lambda=$ $0)$ and may be thought of as a superfield. The bracket $\langle\cdot\rangle_{0}$ picks up the coefficient of $\left(\lambda \gamma^{m} \theta\right)\left(\lambda \gamma^{n} \theta\right)\left(\lambda \gamma^{p} \theta\right)\left(\theta \gamma_{m n p} \theta\right)$ from $\mathcal{K}_{(N)}$ in the cohomology of the left-moving BRST charge $[58,59]$. It will often be convenient to manipulate the full superfield $\mathcal{K}_{(N)}$ rather than its component $\left\langle\mathcal{K}_{(N)}\right\rangle_{0}$ and, by a slight abuse of notation, we shall refer to both as chiral correlators.

The chiral correlator $\mathcal{K}_{(N)}$ is a locally holomorphic $(1,0)$ form in each vertex point $z_{i}$, and is invariant under homology shifts but, in contrast to $\mathcal{I}_{(N)}$ and $\mathcal{F}_{(N)}$, without phase factors,

$$
\begin{aligned}
& \mathcal{K}_{(N)}\left(z_{i}, k_{i}, p^{I}\right)=\mathcal{K}_{(N)}\left(z_{i}+\delta_{i j} \mathfrak{A}_{J}, k_{i}, p^{I}\right) \\
& \mathcal{K}_{(N)}\left(z_{i}, k_{i}, p^{I}\right)=\mathcal{K}_{(N)}\left(z_{i}+\delta_{i j} \mathfrak{B}_{J}, k_{i}, p^{I}-\delta_{J}^{I} k_{j}\right)
\end{aligned}
$$

The anti-chiral correlator $\left\langle\tilde{\mathcal{K}}_{(N)}\right\rangle_{0}$ is expressed analogously in terms of the right-moving polarization vectors $\tilde{\varepsilon}_{i}^{m}$, and right-moving spinors $\tilde{\chi}_{i}^{\alpha}$ for the Type II strings or the rightmoving gauge data for Heterotic strings. The corresponding superfield $\tilde{\mathcal{K}}_{(N)}$ additionally depends on the zero modes of the right-moving spinor fields $\tilde{\theta}^{\alpha}, \tilde{\lambda}^{\alpha}$. As usual, the left- and right-moving Weyl spinors $\theta^{\alpha}, \lambda^{\alpha}$ and $\tilde{\theta}^{\alpha}, \tilde{\lambda}^{\alpha}$ have the same chirality for Type IIB strings, or opposite chirality for Type IIA strings.

The chiral correlator $\mathcal{K}_{(4)}$ is independent of loop momenta, and given by [7],

$$
\mathcal{K}_{(4)}=T_{1,2 \mid 3,4} \Delta(4,1) \Delta(2,3)+T_{1,4 \mid 2,3} \Delta(1,2) \Delta(3,4)
$$

where $\Delta(x, y)=-\Delta(y, x)$ is the standard bi-holomorphic one-form (see appendix A), and the superfield $T_{1,2 \mid 3,4}$ is a function of the momenta $k_{i}^{m}$, polarization vectors $\varepsilon_{i}^{m}$, spinors $\chi_{i}^{\alpha}$, and the zero modes of $\theta^{\alpha}$ and $\lambda^{\alpha}$. The anti-chiral correlator $\tilde{\mathcal{K}}_{(4)}$ is given by the same formula, with $T_{1,2 \mid 3,4}$ replaced by $\tilde{T}_{1,2 \mid 3,4}$ which depends on $k_{i}^{m}, \tilde{\varepsilon}_{i}^{m}, \tilde{\chi}_{i}^{\alpha}, \tilde{\theta}^{\alpha}$ and $\tilde{\lambda}^{\alpha}$.

The chiral correlator $\mathcal{K}_{(5)}$ and its counterpart $\tilde{\mathcal{K}}_{(5)}$ for Type II strings were shown in [12] to be linear in the loop momenta $p^{I}$, and were decomposed as follows,

$$
\begin{aligned}
& \mathcal{K}_{(5)}=\mathcal{W}+2 \pi i \hat{p}_{m}^{I} \mathcal{V}_{I}^{m} \\
& \tilde{\mathcal{K}}_{(5)}=\tilde{\mathcal{W}}+2 \pi i \hat{p}_{m}^{I} \tilde{\mathcal{V}}_{I}^{m}
\end{aligned}
$$

where $\hat{p}^{I}$ is the shifted loop momentum defined by,

$$
\hat{p}^{I}=p^{I}+Y^{I J} \sum_{i=1}^{5} k_{i} \operatorname{Im} \int_{z_{0}}^{z_{i}} \omega_{J}
$$

with $Y^{I J}$ the inverse of the imaginary part $Y_{I J}=\operatorname{Im} \Omega_{I J}$ of the period matrix $\Omega$.

Several equivalent representations of the chiral correlator $\mathcal{K}_{(5)}$ were given in sections 5 and 6 of [12], each one manifesting different properties of the integrand in (2.1). The 
representation in terms of superspace building blocks $T_{1,2,3 \mid 4,5}^{m}$ and $S_{1 ; 2|3| 4,5}$, to be reviewed below, is given by, ${ }^{5}$

$$
\begin{aligned}
\mathcal{V}_{I}^{m} & =T_{1,2,3 \mid 4,5}^{m} \omega_{I}(2) \Delta(3,4) \Delta(5,1)+\operatorname{cycl}(1,2,3,4,5) \\
\mathcal{W} & =\left(\frac{\alpha^{\prime}}{2}\right) \mathcal{Q}_{12}+(1,2 \mid 1,2,3,4,5)
\end{aligned}
$$

The notation $+\operatorname{cycl}(1,2,3,4,5)$ stands for the addition of all cyclic permutations, while $+(i, j \mid 1,2,3,4,5)$ stands for the addition of all ordered choices of $i$ and $j$ from the set $\{1,2,3,4,5\}$ for a total of $\left(\begin{array}{l}5 \\ 2\end{array}\right)=10$ terms. The function $\mathcal{Q}_{12}$ is given by,

$$
\begin{aligned}
\mathcal{Q}_{12}= & -\partial_{1} \mathcal{G}(1,2)\left[S_{1 ; 2|3| 4,5} \Delta(2,4) \Delta(3,5)+S_{1 ; 2|4| 3,5} \Delta(2,3) \Delta(4,5)\right] \\
& -\partial_{2} \mathcal{G}(2,1)\left[S_{2 ; 1|3| 4,5} \Delta(1,4) \Delta(3,5)+S_{2 ; 1|4| 3,5} \Delta(1,3) \Delta(4,5)\right]
\end{aligned}
$$

where $\mathcal{G}(i, j)=\mathcal{G}\left(z_{i}, z_{j}\right)$ is the Arakelov Green function (see appendix A.5 or [31], section 2.4).

While the expression (2.11) is compact, it does not optimally expose the singularities of the correlator at coincident vertex positions $z_{1} \rightarrow z_{2}$. This is achieved by the alternative representation,

$$
\begin{aligned}
\mathcal{Q}_{12}= & -\partial_{1} \mathcal{G}(1,2)\left[T_{12,3 \mid 4,5} \Delta(2,4) \Delta(3,5)+T_{12,4 \mid 3,5} \Delta(2,3) \Delta(4,5)\right] \\
& -S_{2 ; 1|3| 4,5}\left[\partial_{1} \mathcal{G}(1,2) \Delta(2,4) \Delta(3,5)+\partial_{2} \mathcal{G}(2,1) \Delta(1,4) \Delta(3,5)\right] \\
& -S_{2 ; 1|4| 3,5}\left[\partial_{1} \mathcal{G}(1,2) \Delta(2,3) \Delta(4,5)+\partial_{2} \mathcal{G}(2,1) \Delta(1,3) \Delta(4,5)\right]
\end{aligned}
$$

where the singularity as $z_{1} \rightarrow z_{2}$ is contained entirely in the first line, while the second and third lines are manifestly regular due to the cancellation of the poles from $\partial_{1} \mathcal{G}(1,2)$ and $\partial_{2} \mathcal{G}(1,2)$. In particular, (2.12) makes it manifest that the residues of kinematic poles in the integrated amplitude will only feature permutations of $\left|T_{12,3 \mid 4,5}\right|^{2}$.

When discussing the difference between Type IIA and Type IIB amplitudes in sections 4.3 and 4.4, a third representation of the correlator will become convenient, given in terms of

$$
\begin{aligned}
\widehat{\mathcal{V}}_{I}^{m} & =C_{1,2,3 \mid 4,5}^{m} \omega_{I}(2) \Delta(3,4) \Delta(5,1)+\operatorname{cycl}(1,2,3,4,5) \\
\widehat{\mathcal{W}} & =\left(\frac{\alpha^{\prime}}{2}\right) \widehat{\mathcal{Q}}_{12}+(1,2 \mid 1,2,3,4,5)
\end{aligned}
$$

with

$$
\begin{aligned}
\widehat{\mathcal{Q}}_{12}= & -s_{12} \partial_{1} \mathcal{G}(1,2)\left[C_{1 ; 2|3| 4,5} \Delta(2,4) \Delta(3,5)+C_{1 ; 2|4| 3,5} \Delta(2,3) \Delta(4,5)\right] \\
& -s_{12} \partial_{2} \mathcal{G}(2,1)\left[C_{2 ; 1|3| 4,5} \Delta(1,4) \Delta(3,5)+C_{2 ; 1|4| 3,5} \Delta(1,3) \Delta(4,5)\right]
\end{aligned}
$$

Here, the superfields $C_{1,2,3 \mid 4,5}^{m}$ and $C_{1 ; 2|3| 4,5}$ are non-local, but manifestly BRST-closed, building blocks to be described below. The correlators of (2.13) can be shown to be equivalent to (2.10) after substituting the relations to be given below in (2.24) and discarding

\footnotetext{
${ }^{5}$ For reasons to become clear in section 4 , we have restored a factor of $\frac{\alpha^{\prime}}{2}$ in order to match with the conventions of [8], see e.g. (5.40) of that reference.
} 
total derivatives. ${ }^{6}$ Note that, in both of these representations, $\mathcal{Q}_{12}$ and $\widehat{\mathcal{Q}}_{12}$ are totally symmetric in the omitted labels $3,4,5$ due to the symmetries of the building blocks as well as $\Delta(2,3) \Delta(4,5)+\operatorname{cyc}(3,4,5)=0$.

Similar expressions are valid for the right-moving parts $\tilde{\mathcal{W}}$ and $\tilde{\mathcal{V}}$, with $T_{1,2,3 \mid 4,5}^{m}$ and $S_{1 ; 2|3| 4,5}$ replaced by their counterparts $\tilde{T}_{1,2,3 \mid 4,5}^{m}$ and $\tilde{S}_{1 ; 2|3| 4,5}$ depending on the zero modes of $\tilde{\theta}^{\alpha}$ and $\tilde{\lambda}^{\alpha}$ (with the usual chirality flip for Type IIA).

\subsection{Scalar and vector superspace building blocks}

To complete the definition of the integrands, it remains to specify the superspace constituents referred to above as "building blocks". These are kinematic expressions in pure spinor superspace, constructed using the multiparticle formalism of the standard superfields of ten-dimensional SYM [61].

\subsubsection{Local building blocks}

The four-point scalar block $T_{1,2 \mid 3,4}$ was constructed in $[7,57]$ and satisfies,

$$
\begin{aligned}
Q T_{1,2 \mid 3,4} & =0 \\
T_{1,2 \mid 3,4} & =T_{2,1 \mid 3,4}=T_{3,4 \mid 1,2} \\
T_{1,2 \mid 3,4} & =-T_{1,3 \mid 4,2}-T_{1,4 \mid 2,3}
\end{aligned}
$$

where $Q=\lambda^{\alpha} D_{\alpha}$ is the BRST operator of the pure spinor formalism [58] with,

$$
D_{\alpha}=\frac{\partial}{\partial \theta^{\alpha}}+\frac{1}{2}\left(\gamma^{m} \theta\right)_{\alpha} \frac{\partial}{\partial x^{m}}
$$

The derivative with respect to $x^{m}$ acts on the plane-wave factor $e^{i k \cdot x}$ of each superfield to produce a factor of $i k_{m}$. The properties (2.15) along with the antisymmetry of $\Delta(i, j)$ ensure the invariance of (2.7) under permutations of the 4 external states.

The five-point vector block $T_{1,2,3 \mid 4,5}^{m}$ was constructed in [14] so as to satisfy,

$$
Q T_{1,2,3 \mid 4,5}^{m}=i k_{1}^{m} V_{1} T_{2,3 \mid 4,5}+i k_{2}^{m} V_{2} T_{3,1 \mid 4,5}+i k_{3}^{m} V_{3} T_{1,2 \mid 4,5}
$$

as well as the following symmetry relations,

$$
\begin{aligned}
& T_{1,2,3 \mid 4,5}^{m}=T_{3,4,5 \mid 1,2}^{m}+T_{2,4,5 \mid 1,3}^{m}+T_{1,4,5 \mid 2,3}^{m} \\
& T_{1,2,3 \mid 4,5}^{m}=T_{1,3,2 \mid 4,5}^{m}=T_{2,1,3 \mid 4,5}^{m}=T_{1,2,3 \mid 5,4}^{m}
\end{aligned}
$$

where $V_{i}$ are the BRST-closed one-particle unintegrated vertex operators. The relations (2.18) ensure that $\mathcal{V}_{I}^{m}$ in (2.10) is invariant under permutations of the five external legs.

In addition, a scalar superfield $T_{12,3 \mid 4,5}$ was constructed in [14] using two-particle superfields obeying,

$$
Q T_{12,3 \mid 4,5}=s_{12}\left(V_{1} T_{2,3 \mid 4,5}-V_{2} T_{1,3 \mid 4,5}\right)
$$

\footnotetext{
${ }^{6}$ The correlators of $(2.10)$ and (2.13) may be formally related by the substitution rule $T_{1,2,3 \mid 4,5}^{m} \rightarrow$ $C_{1,2,3 \mid 4,5}^{m}$ and $S_{1 ; 2|3| 4,5} \rightarrow s_{12} C_{1 ; 2|3| 4,5}$. This rule mimics similar manipulations observed at one loop [60].
} 
as well as $T_{12,3 \mid 4,5}=T_{12,3 \mid 5,4}$ and the "Jacobi" symmetry,

$$
T_{12,3 \mid 4,5}+T_{12,4 \mid 5,3}+T_{12,5 \mid 3,4}=0
$$

Finally, the five-point scalar blocks in (2.11) are given by [12],

$$
S_{1 ; 2|3| 4,5}=\frac{1}{2}\left(i\left(k_{1}^{m}+k_{2}^{m}-k_{3}^{m}\right) T_{1,2,3 \mid 4,5}^{m}+T_{12,3 \mid 4,5}+T_{13,2 \mid 4,5}+T_{23,1 \mid 4,5}\right)
$$

and satisfy,

$$
Q S_{1 ; 2|3| 4,5}=s_{12} V_{1} T_{2,3 \mid 4,5}, \quad S_{1 ; 2|3| 4,5}=S_{1 ; 2|3| 5,4}, \quad T_{12,3 \mid 4,5}=S_{1 ; 2|3| 4,5}-S_{2 ; 1|3| 4,5}
$$

Furthermore, we have the following relations between permutations of (2.21),

$$
\begin{array}{r}
S_{1 ; 2|3| 4,5}+S_{1 ; 2|4| 5,3}+S_{1 ; 2|5| 3,4} \cong 0 \\
S_{1 ; 2|3| 4,5}+S_{1 ; 3|2| 4,5}+S_{1 ; 4|5| 2,3}+S_{1 ; 5|4| 2,3} \cong 0
\end{array}
$$

where $\cong$ denotes an equality in the BRST cohomology. Importantly, the bosonic components of the vector building blocks $T_{1,2,3 \mid 4,5}^{m}$ are proportional to $k^{6} \varepsilon^{5}$ while those of the scalar blocks $T_{12,3 \mid 4,5}$ and $S_{1 ; 2|3| 4,5}$ are proportional to $k^{7} \varepsilon^{5}$, where $\varepsilon$ represents the SYM

polarization vector. As a consequence, gravitational components of $T_{1,2,3 \mid 4,5}^{m} \tilde{T}_{1,2,3 \mid 4,5}^{m}$ and $T_{12,3 \mid 4,5} \tilde{T}_{12,3 \mid 4,5} / k_{1} \cdot k_{2}$ have the mass dimension of $D^{2} \mathcal{R}^{5}$.

\subsubsection{Non-local building blocks}

Besides the above building blocks, which are polynomials in external momenta, it will be useful to introduce the non-local combinations introduced in section 5.4 of [12],

$$
\begin{aligned}
C_{1 ; 3|4| 2,5}= & \frac{1}{4}\left(\frac{3 S_{1 ; 3|4| 2,5}}{s_{13}}-\frac{S_{1 ; 4|3| 2,5}}{s_{14}}-\frac{S_{1 ; 2|5| 3,4}}{s_{12}}-\frac{S_{1 ; 5|2| 3,4}}{s_{15}}\right) \\
C_{5,1,2 \mid 3,4}^{m}= & T_{5,1,2 \mid 3,4}^{m}-\frac{i}{4} k_{1}^{m}\left(\frac{S_{1 ; 2|5| 3,4}}{s_{12}}+\frac{S_{1 ; 5|2| 3,4}}{s_{15}}+\frac{S_{1 ; 3|4| 2,5}}{s_{13}}+\frac{S_{1 ; 4|3| 2,5}}{s_{14}}\right) \\
& -\frac{i}{4} k_{2}^{m}\left(\frac{S_{2 ; 1|5| 3,4}}{s_{12}}+\frac{S_{2 ; 5|1| 3,4}}{s_{25}}+\frac{S_{2 ; 3|4| 1,5}}{s_{23}}+\frac{S_{2 ; 4|3| 1,5}}{s_{24}}\right) \\
& -\frac{i}{4} k_{5}^{m}\left(\frac{S_{5 ; 1|2| 3,4}}{s_{15}}+\frac{S_{5 ; 2|1| 3,4}}{s_{25}}+\frac{S_{5 ; 3|4| 1,2}}{s_{35}}+\frac{S_{5 ; 4|3| 1,2}}{s_{45}}\right)
\end{aligned}
$$

that are manifestly BRST invariant

$$
\begin{aligned}
& Q C_{5,1,2 \mid 3,4}^{m}=0 \\
& Q C_{1 ; 3|4| 2,5}=0
\end{aligned}
$$

In addition, they satisfy the following relations [12],

$$
\begin{aligned}
i k_{2}^{m} C_{5,1,2 \mid 3,4}^{m} & =s_{12} C_{1 ; 2|5| 3,4}+s_{25} C_{5 ; 2|1| 3,4} \\
i k_{3}^{m} C_{5,1,2 \mid 3,4}^{m} & \cong s_{13} C_{1 ; 3|4| 2,5}+s_{23} C_{2 ; 3|4| 1,5}+s_{35} C_{5 ; 3|4| 1,2} \\
0 & \cong s_{12} C_{2 ; 1|5| 3,4}+s_{25} C_{2 ; 5|1| 3,4}+s_{23} C_{2 ; 3|4| 1,5}+s_{24} C_{2 ; 4|3| 1,5} \\
0 & \cong C_{2 ; 1|5| 3,4}+C_{2 ; 1|4| 5,3}+C_{2 ; 1|3| 4,5} \\
0 & =C_{2 ; 1|5| 3,4}-C_{2 ; 1|5| 4,3}
\end{aligned}
$$


Importantly, the invariants $C_{1,2,3 \mid 4,5}^{m}$ and $C_{1 ; 2|3| 4,5}$, which we call "two-loop BRST invariants", can be rewritten in terms of similar BRST invariants $C_{1 \mid 2,3,4,5}^{m}$ and $C_{1 \mid 23,4,5}[60,61]$ (the "one-loop BRST invariants") which occur ${ }^{7}$ in the integrand of the one-loop five-point amplitude [35, 62]. Using the components $\left\langle C_{1 \mid 2,3,4,5}^{m}\right\rangle_{0}$ and $\left\langle C_{1 \mid 23,4,5}\right\rangle_{0}$ available for download from [63], one finds [12],

$$
\begin{aligned}
C_{1,2,3 \mid 4,5}^{m} \cong & -16 s_{45} C_{1 \mid 2,3,4,5}^{m}+8\left(k_{4}^{m}-k_{5}^{m}\right) s_{45} C_{1 \mid 45,2,3} \\
& +4 k_{2}^{m}\left(s_{45}\left(C_{1 \mid 24,3,5}+C_{1 \mid 25,3,4}\right)+\left(s_{13}+s_{23}\right) C_{1 \mid 23,4,5}\right) \\
& \left.+4 k_{3}^{m}\left(s_{45}\left(C_{1 \mid 34,2,5}+C_{1 \mid 35,2,4}\right)-\left(s_{12}+s_{23}\right) C_{1 \mid 23,4,5}\right)\right) \\
& -4\left(k_{1}^{m}+k_{2}^{m}+k_{3}^{m}\right)\left(s_{24} C_{1 \mid 24,3,5}+s_{25} C_{1 \mid 25,3,4}+(2 \leftrightarrow 3)\right)
\end{aligned}
$$

and

$$
C_{1 ; 2|3| 4,5} \cong 4\left(s_{24} C_{1 \mid 24,3,5}+s_{25} C_{1 \mid 25,3,4}+s_{34} C_{1 \mid 34,2,5}+s_{35} C_{1 \mid 35,2,4}-2 s_{23} C_{1 \mid 23,4,5}\right)
$$

In turn, the components of the one-loop BRST invariants can be expressed as combinations of color-ordered tree amplitudes ${ }^{8}[60,61]$,

$$
\begin{aligned}
\left\langle C_{1 \mid 23,4,5}\right\rangle_{0} & =s_{45}\left[s_{34} A_{\mathrm{YM}}(1,2,3,4,5)-s_{24} A_{\mathrm{YM}}(1,3,2,4,5)\right] \\
0 & =\left\langle i k_{2}^{m} C_{1 \mid 2,3,4,5}^{m}+\left[s_{23} C_{1 \mid 23,4,5}+(3 \leftrightarrow 4,5)\right]\right\rangle_{0}
\end{aligned}
$$

These relations will become useful in section 5 when comparing our two-loop results with one-loop and tree-level amplitudes.

\subsection{Effective rules for bosonic components}

The bosonic components ${ }^{9}$ of the building blocks $T_{1,2,3 \mid 4,5}^{m}$ and $T_{12,3 \mid 4,5}$ in pure spinor superspace are available for download from the website [63]. However, the expressions from [63] involve unpleasant rational factors such as $\frac{13}{7}$ within individual $\left\langle T_{1,2,3 \mid 4,5}^{m}\right\rangle_{0}$ or $\left\langle T_{12,3 \mid 4,5}\right\rangle_{0}$, which drop out from BRST invariants. These factors come from an implicit choice of contact terms, which is far from being canonical nor optimal.

In order to streamline the expressions for the bosonic components of the local building blocks and facilitate the comparison with the RNS computation [15], we shall now give an alternative description of the correlators in [12]. The key quantities are the effective

\footnotetext{
${ }^{7}$ Note that in [35] the object called $C_{1,2,3,4,5}^{m} \tilde{C}_{1,2,3,4,5}^{m}$ is a shorthand for the leading-order contributions from the correlator and should not be confused with the holomorphic square of $C_{1 \mid 2,3,4,5}^{m}$.

${ }^{8}$ We have $k_{j} \rightarrow i k_{j}$ and different conventions for $s_{i j}$ in comparison to the definitions in [60].

${ }^{9}$ With the techniques of [64] to perform the zero-mode integrals over $\lambda^{\alpha}, \theta^{\alpha}$, one can obtain direct access to the polarization dependence of the five-point amplitudes in string and field-theory for any combination of external bosons and fermions.
} 
components $T_{1,2,3 \mid 4,5}^{m, \mathrm{eff}}, T_{12,3 \mid 4,5}^{\mathrm{eff}}$ and $S_{1 ; 2|3| 4,5}^{\mathrm{eff}}$ defined by

$$
\begin{aligned}
T_{1,2,3 \mid 4,5}^{m, \mathrm{eff}}= & 8\left(k_{4} \cdot k_{5}\right)\left[\varepsilon_{1}^{m} t_{8}\left(f_{2}, f_{3}, f_{4}, f_{5}\right)+(1 \leftrightarrow 2,3,4,5)\right] \\
& +4 i\left[k_{1}^{m}\left(R_{1 ; 2 \mid 3,4,5}+R_{1 ; 3 \mid 2,4,5}\right)+(1 \leftrightarrow 2,3)\right] \\
& +8 i k_{4}^{m} R_{4 ; 5 \mid 1,2,3}+8 i k_{5}^{m} R_{5 ; 4 \mid 1,2,3}-4\left(k_{4} \cdot k_{5}\right) \epsilon_{10}^{m}\left(\varepsilon_{1}, f_{2}, f_{3}, f_{4}, f_{5}\right) \\
T_{12,3 \mid 4,5}^{\mathrm{eff}}= & \left(8 k_{4} \cdot k_{5}-4 k_{1} \cdot k_{2}\right)\left(R_{1 ; 2 \mid 3,4,5}-R_{2 ; 1 \mid 3,4,5}\right)+4 k_{1} \cdot k_{2}\left(R_{2 ; 3 \mid 1,4,5}-R_{1 ; 3 \mid 2,4,5}\right) \\
S_{1 ; 2|3| 4,5}^{\mathrm{eff}}= & \left(8 k_{4} \cdot k_{5}-4 k_{1} \cdot k_{2}\right) R_{1 ; 2 \mid 3,4,5}-4 k_{1} \cdot k_{2} R_{1 ; 3 \mid 2,4,5} \\
& +8\left(k_{3} \cdot k_{4} R_{4 ; 5 \mid 1,2,3}-k_{4} \cdot k_{5} R_{4 ; 3 \mid 1,2,5}\right) \\
& +8\left(k_{3} \cdot k_{5} R_{5 ; 4 \mid 1,2,3}-k_{4} \cdot k_{5} R_{5 ; 3 \mid 1,2,4}\right)
\end{aligned}
$$

which are composed of

$$
\begin{aligned}
R_{1 ; 2 \mid 3,4,5} & =i\left(\varepsilon_{1} \cdot k_{2}\right) t_{8}\left(f_{2}, f_{3}, f_{4}, f_{5}\right)-\frac{i}{2} t_{8}\left(\left[f_{1}, f_{2}\right], f_{3}, f_{4}, f_{5}\right) \\
t_{8}\left(f_{2}, f_{3}, f_{4}, f_{5}\right) & =\operatorname{tr}\left(f_{2} f_{3} f_{4} f_{5}\right)-\frac{1}{4} \operatorname{tr}\left(f_{2} f_{3}\right) \operatorname{tr}\left(f_{4} f_{5}\right)+\operatorname{cyc}(3,4,5)
\end{aligned}
$$

with Lorentz traces $\operatorname{tr}(\ldots)$, linearized field strength $f_{j}^{m n}=\varepsilon_{j}^{m} k_{j}^{n}-\varepsilon_{j}^{n} k_{j}^{m}$ and its commutators $\left[f_{1}, f_{2}\right]^{m n}=f_{1}^{m p} f_{2}^{p n}-f_{2}^{m p} f_{1}^{p n}$. As will be explained below, the bosonic components of the two-loop five-point amplitude are unchanged when performing the replacement

$$
T_{1,2,3 \mid 4,5}^{m} \rightarrow T_{1,2,3 \mid 4,5}^{m, \mathrm{eff}}, \quad T_{12,3 \mid 4,5} \rightarrow T_{12,3 \mid 4,5}^{\mathrm{eff}}, \quad S_{1 ; 2|3| 4,5} \rightarrow S_{1 ; 2|3| 4,5}^{\mathrm{eff}}
$$

in all terms of the correlator (2.8) and dropping the zero-mode brackets $\langle\ldots\rangle_{0}$ in the chiral amplitude (2.2).

\subsubsection{Symmetries and relations of the effective components}

The effective replacement rules (2.32) are well-defined at the level of $\left\langle\mathcal{K}_{(5)}\right\rangle_{0}$ since all of $T_{1,2,3 \mid 4,5}^{\text {m,eff }}, T_{12,3 \mid 4,5}^{\text {eff }}, S_{1 ; 2|3| 4,5}^{\text {eff }}$ given by (2.30) inherit the symmetry relations of the superfields $T_{1,2,3 \mid 4,5}^{m}, T_{12,3 \mid 4,5}, S_{1 ; 2|3| 4,5}$ in the BRST cohomology. This is a consequence of the symmetry of $t_{8}$,

$$
R_{1 ; 2 \mid 3,4,5}=R_{1 ; 2 \mid 4,3,5}=R_{1 ; 2 \mid 3,5,4}
$$

as well as momentum conservation, transversality of $\varepsilon_{i}$, and the relation $\operatorname{tr}\left(f_{1} f_{2} f_{3} f_{4} f_{5}\right)=$ $-\operatorname{tr}\left(f_{1} f_{5} f_{4} f_{3} f_{2}\right)$ used in $(2.31)$,

$$
R_{1 ; 2 \mid 3,4,5}+R_{1 ; 3 \mid 2,4,5}+R_{1 ; 4 \mid 2,3,5}+R_{1 ; 5 \mid 2,3,4}=0
$$

as well as the identity,

$$
i k_{1}^{m}\left[\varepsilon_{1}^{m} t_{8}\left(f_{2}, f_{3}, f_{4}, f_{5}\right)+(1 \leftrightarrow 2,3,4,5)\right]=R_{2 ; 1 \mid 3,4,5}+R_{3 ; 1 \mid 2,4,5}+R_{4 ; 1 \mid 2,3,5}+R_{5 ; 1 \mid 2,3,4}
$$


where the commutators $\left[f_{i}, f_{j}\right]$ all drop out from the right-hand side. These basic properties imply that the effective components in (2.30) obey

$$
\begin{array}{ll}
T_{12,3 \mid 4,5}^{\mathrm{eff}}=T_{12,3 \mid 5,4}^{\mathrm{eff}} & 0=T_{12,3 \mid 4,5}^{\mathrm{eff}}+T_{12,4 \mid 5,3}^{\mathrm{eff}}+T_{12,5 \mid 3,4}^{\mathrm{eff}} \\
S_{1 ; 2|3| 4,5}^{\mathrm{eff}}=S_{1 ; 2|3| 5,4}^{\mathrm{eff}} & 0=S_{1 ; 2|3| 4,5}^{\mathrm{eff}}+S_{1 ; 2|4| 5,3}^{\mathrm{eff}}+S_{1 ; 2|5| 3,4}^{\mathrm{eff}}
\end{array}
$$

as well as

$$
\begin{aligned}
0 & =S_{1 ; 2|3| 4,5}^{\mathrm{eff}}+S_{1 ; 3|2| 4,5}^{\mathrm{eff}}+S_{1 ; 4|5| 2,3}^{\mathrm{eff}}+S_{1 ; 5|4| 2,3}^{\mathrm{eff}} \\
T_{1,2,3 \mid 4,5}^{m, \mathrm{eff}} & =T_{3,4,5 \mid 1,2}^{m, \mathrm{eff}}+T_{2,4,5 \mid 1,3}^{m, \mathrm{eff}}+T_{1,4,5 \mid 2,3}^{m, \text { eff }} \\
T_{1,2,3 \mid 4,5}^{m, \mathrm{eff}} & =T_{1,3,2 \mid 4,5}^{m, \mathrm{eff}}=T_{2,1,3 \mid 4,5}^{m, \mathrm{eff}}=T_{1,2,3 \mid 5,4}^{m, \mathrm{eff}}
\end{aligned}
$$

and are related by

$$
\begin{aligned}
S_{1 ; 2|3| 4,5}^{\mathrm{eff}} & =\frac{1}{2}\left[i\left(k_{12}^{m}-k_{3}^{m}\right) T_{1,2,3 \mid 4,5}^{m, \mathrm{eff}}+T_{12,3 \mid 4,5}^{\mathrm{eff}}+T_{13,2 \mid 4,5}^{\mathrm{eff}}+T_{23,1 \mid 4,5}^{\mathrm{eff}}\right] \\
T_{12,3 \mid 4,5}^{\mathrm{eff}} & =S_{1 ; 2|3| 4,5}^{\mathrm{eef}}-S_{2 ; 1|3| 4,5}^{\mathrm{eff}} \\
i k_{1}^{m} T_{1,2,3 \mid 4,5}^{m, \mathrm{eff}} & =S_{2 ; 1|3| 4,5}^{\mathrm{eff}}+S_{3 ; 1|2| 4,5}^{\mathrm{eff}} \\
i k_{5}^{m} T_{1,2,3 \mid 4,5}^{m, \mathrm{eff}} & =S_{1 ; 5|4| 2,3}^{\mathrm{eff}}+S_{2 ; 5|4| 1,3}^{\mathrm{eff}}+S_{3 ; 5|4| 1,2}^{\mathrm{eff}} \\
i k_{3}^{m}\left(T_{1,2,3 \mid 4,5}^{m, \text { eff }}+T_{3,4,5 \mid 1,2}^{m, \mathrm{eff}}\right) & =T_{13,2 \mid 4,5}^{\mathrm{eff}}+T_{23,1 \mid 4,5}^{\mathrm{eff}}-T_{34,5 \mid 1,2}^{\mathrm{eff}}-T_{35,4 \mid 1,2}^{\mathrm{eff}}
\end{aligned}
$$

Hence, any relation among the superfields in the BRST cohomology — see e.g. (2.18) to $(2.23)$ - is preserved by the transition (2.32) to effective bosonic components.

In fact, we have checked that the bosonic components of any BRST-invariant quantity composed from the building blocks reviewed above can be obtained by using their "effective" versions,

$$
\left(S_{a ; b|c| d, e}, T_{a b, c \mid d, e}, T_{a, b, c \mid d, e}^{m}\right) \rightarrow\left(S_{a ; b|c| d, e}^{\mathrm{eff}}, T_{a b, c \mid d, e}^{\mathrm{eff}}, T_{a, b, c \mid d, e}^{m, \mathrm{eff}}\right)
$$

This includes all representations of the genus-two correlator (2.10) since they obviously are BRST invariant.

\subsubsection{Effective BRST invariants and correlators}

The effective bosonic components (2.30) not only preserve the relations of their superspace prototypes but also the two-loop BRST invariants (2.24): one can check from the results on the website [63] that,

$$
\begin{aligned}
-\left.2880\left\langle C_{1 ; 3|4| 2,5}\right\rangle_{0}\right|_{\mathrm{bos}}= & \frac{1}{4}\left(\frac{3 S_{1 ; 3|4| 2,5}^{\mathrm{eff}}}{s_{13}}-\frac{S_{1 ; 4|3| 2,5}^{\mathrm{eff}}}{s_{14}}-\frac{S_{1 ; 2|5| 3,4}^{\mathrm{eff}}}{s_{12}}-\frac{S_{1 ; 5|2| 3,4}^{\mathrm{eff}}}{s_{15}}\right) \\
-\left.2880\left\langle C_{5,1,2 \mid 3,4}^{m}\right\rangle_{0}\right|_{\mathrm{bos}}= & T_{5,1,2 \mid 3,4}^{m, \mathrm{eff}}-\frac{i}{4} k_{1}^{m}\left(\frac{S_{1 ; 2|5| 3,4}^{\mathrm{eff}}}{s_{12}}+\frac{S_{1 ; 5|2| 3,4}^{\text {eff }}}{s_{15}}+\frac{S_{1 ; 3|4| 2,5}^{\mathrm{eff}}}{s_{13}}+\frac{S_{1 ; 4|3| 2,5}^{\mathrm{eff}}}{s_{14}}\right) \\
& -\frac{i}{4} k_{2}^{m}\left(\frac{S_{2 ; 1|5| 3,4}^{\mathrm{eff}}}{s_{12}}+\frac{S_{2 ; 5|1| 3,4}^{\mathrm{eff}}}{s_{25}}+\frac{S_{2 ; 3|4| 1,5}^{\mathrm{eff}}}{s_{23}}+\frac{S_{2 ; 4|3| 1,5}^{\mathrm{eff}}}{s_{24}}\right) \\
& -\frac{i}{4} k_{5}^{m}\left(\frac{S_{5 ; 1|2| 3,4}^{\mathrm{eff}}}{s_{15}}+\frac{S_{5 ; 2|1| 3,4}^{\mathrm{eff}}}{s_{25}}+\frac{S_{5 ; 3|4| 1,2}^{\mathrm{eff}}}{s_{35}}+\frac{S_{5 ; 4|3| 1,2}^{\mathrm{eff}}}{s_{45}}\right)
\end{aligned}
$$


Since the chiral amplitude is expressible in terms of the BRST invariants (2.24) [12], its bosonic components can be equivalently expressed in terms of the effective components. One can then pass back to a local representation by repeating the integration-by-parts manipulations in section 5.4 of the reference with effective components in the place of the superfields: the bosonic components of the string amplitude are unchanged when $\left\langle\mathcal{K}_{(5)}\right\rangle_{0}$ in the chiral amplitude (2.2) is replaced by

$$
\mathcal{K}_{(5)}^{\mathrm{eff}}=\omega_{I}(2) \Delta(3,4) \Delta(5,1) \mathcal{K}_{1,2,3 \mid 4,5}^{I, \mathrm{eff}}+\operatorname{cycl}(1,2,3,4,5)
$$

where

$$
\begin{aligned}
\mathcal{K}_{1,2,3 \mid 4,5}^{I, \mathrm{eff}}= & 2 \pi p_{m}^{I} T_{1,2,3 \mid 4,5}^{m, \mathrm{eff}}-g_{2,3}^{I} T_{23,1 \mid 4,5}^{\mathrm{eff}}-g_{2,1}^{I} T_{21,3 \mid 4,5}^{\mathrm{eff}}-g_{3,1}^{I} T_{31,2 \mid 4,5}^{\mathrm{eff}} \\
& -g_{2,4}^{I} S_{2 ; 4|5| 1,3}^{\mathrm{eff}}-g_{3,4}^{I} S_{3 ; 4|5| 2,1}^{\mathrm{eff}}-g_{1,4}^{I} S_{1 ; 4|5| 2,3}^{\mathrm{eff}} \\
& -g_{2,5}^{I} S_{2 ; 5|4| 3,1}^{\mathrm{eff}}-g_{3,5}^{I} S_{3 ; 5|4| 2,1}^{\mathrm{eff}}-g_{1,5}^{I} S_{1 ; 5|4| 2,3}^{\mathrm{eff}}
\end{aligned}
$$

and (for some odd spin structure $\nu$ whose choice is immaterial for (2.41))

$$
g_{i, j}^{I}=\frac{\partial}{\partial \zeta_{I}} \ln \vartheta[\nu](\zeta \mid \Omega), \quad \zeta_{I}=\int_{z_{j}}^{z_{i}} \omega_{I}
$$

Since the effective components inherit all the relations of the superfields, one can also adapt the representation (2.8) in terms of $\hat{p}^{I}$ and the Arakelov Green function to the effective components,

$$
\begin{aligned}
\mathcal{K}_{(5)}^{\text {eff }}= & 2 \pi i \hat{p}_{m}^{I}\left[T_{1,2,3 \mid 4,5}^{m, \text { eff }} \omega_{I}(2) \Delta(3,4) \Delta(5,1)+\operatorname{cycl}(1,2,3,4,5)\right] \\
& +\left(\frac{\alpha^{\prime}}{2}\right)\left[\mathcal{Q}_{12}^{\text {eff }}+(1,2 \mid 1,2,3,4,5)\right] \\
\mathcal{Q}_{12}^{\text {eff }}= & -\partial_{1} \mathcal{G}(1,2)\left[S_{1 ; 2|3| 4,5}^{\text {eff }} \Delta(2,4) \Delta(3,5)+S_{1 ; 2|4| 3,5}^{\text {eff }} \Delta(2,3) \Delta(4,5)\right] \\
& -\partial_{2} \mathcal{G}(2,1)\left[S_{2 ; 1|3| 4,5}^{\text {eff }} \Delta(1,4) \Delta(3,5)+S_{2 ; 1|4| 3,5}^{\text {eff }} \Delta(1,3) \Delta(4,5)\right]
\end{aligned}
$$

\subsection{Assembling and expanding}

After performing the Gaussian integral over loop momenta, the amplitude (2.1) becomes,

$$
\mathcal{A}_{(N)}^{\text {genus }-2}=\delta(k) \mathcal{N}_{(N)} \int_{\mathcal{M}_{2}} d \mu_{2} \mathcal{B}_{(N)}\left(k_{i} \mid \Omega\right)
$$

where $d \mu_{2}$ is the $\operatorname{Sp}(4, \mathbb{R})$ invariant measure on the Siegel upper-half plane, normalized as in $[30,65]$,

$$
d \mu_{2}=\frac{\left|d^{3} \Omega\right|^{2}}{(\operatorname{det} Y)^{3}} \quad \mathrm{Vol}_{2}=\int_{\mathcal{M}_{2}} d \mu_{2}=\frac{2^{2} \pi^{3}}{3^{3} 5}
$$

The integrand $\mathcal{B}_{(N)}\left(k_{i} \mid \Omega\right)$ (which also depends on the polarizations of the external particles, which we do not exhibit here) is given by an integral over $\Sigma^{N}$ and over the zero modes of $\theta^{\alpha}, \tilde{\theta}^{\alpha}, \lambda^{\alpha}, \tilde{\lambda}^{\alpha}$. For $N=4$, the Gaussian integral over $p^{I}$ leads to (see (2.7) for $\mathcal{K}_{(4)}$ )

$$
\mathcal{B}_{(4)}\left(k_{i} \mid \Omega\right)=\int_{\Sigma^{4}} \frac{\mathrm{KN}_{(4)}}{(\operatorname{det} Y)^{2}}\left\langle\mathcal{K}_{(4)} \tilde{\mathcal{K}}_{(4)}\right\rangle_{0}
$$


where $\mathrm{KN}_{(N)}$ is the full Koba-Nielsen factor (as opposed to the chiral one $\mathcal{I}_{(N)}$ in $(2.3)$ ),

$$
\mathrm{KN}_{(N)}\left(k_{i} \mid \Omega\right)=\prod_{1 \leq i<j \leq N} e^{s_{i j} \mathcal{G}\left(z_{i}, z_{j} \mid \Omega\right)}
$$

and $\mathcal{G}\left(z_{i}, z_{j} \mid \Omega\right)$ is the Arakelov Green function (see appendix A). For $N=5$, the integral over loop momenta contains additional terms arising from integrating a bilinear term in loop momenta between left and right movers,

$$
\mathcal{B}_{(5)}\left(k_{i} \mid \Omega\right)=\frac{1}{i \pi} \int_{\Sigma^{5}} \frac{\mathrm{KN}_{(5)}}{(\operatorname{det} Y)^{2}}\left\langle\mathcal{W} \tilde{\mathcal{W}}-\left(\frac{\alpha^{\prime}}{2}\right) \pi Y^{I J} \mathcal{V}_{I}^{m} \tilde{\mathcal{V}}_{J}^{m}\right\rangle_{0}
$$

For brevity, we shall denote the two terms in the angled bracket by $\left\langle|\mathcal{W}|^{2}-\pi\left|\mathcal{V}_{I}^{m}\right|^{2}\right\rangle_{0}$. Upon integration over $\Sigma^{5},(2.49)$ is unchanged when all of $\mathcal{W}, \mathcal{V}_{I}^{m}$ and the corresponding right-movers are replaced by their manifestly BRST invariant counterparts $\widehat{\mathcal{W}}, \widehat{\mathcal{V}}_{I}^{m}$ in (2.13) and (2.11). While the manifestly local or BRST invariant superspace representations of (2.49) apply to any combination of external bosons and fermions, their NSNS components can be alternatively rewritten by replacing the various building blocks within $\mathcal{W}$ and $\mathcal{V}_{I}^{m}$ by their "effective" versions. This form will be useful in the discussion of Type IIA components in section 4.4.

By construction, both (2.47) and (2.49) are invariant under modular transformations of $\Omega$, and can therefore be meaningfully integrated against the invariant measure in (2.45) over the moduli space $\mathcal{M}_{2}$, realized as a fundamental domain of the action of $\operatorname{Sp}(4, \mathbb{Z})$ on the Siegel upper half-space. For Type II strings compactified on a torus $T^{d}$, the measure in (2.45) is multiplied by the Siegel-Narain theta series $\Gamma_{d, d, 2}[66]$, which is modular invariant by itself.

The main goal of this paper will be to analyze the low energy expansion of the five-point integrand (2.49) in powers of the kinematical invariants $s_{i j}$. The dependence on external momenta arises explicitly through the Koba-Nielsen factor (2.48), and through permutations of the building blocks $\left|T_{1,2,3 \mid 4,5}^{m}\right|^{2}$ of dimension $D^{2} \mathcal{R}^{5}$ and $\left|T_{12,3 \mid 4,5}\right|^{2},\left|S_{1 ; 2|3| 4,5}\right|^{2}$ of dimension $D^{4} \mathcal{R}^{5}$. While the integrals multiplying $\left|T_{1,2,3 \mid 4,5}^{m}\right|^{2}$ and $\left|S_{1 ; 2|3| 4,5}\right|^{2}$ in the representation (2.12) are convergent and analytic as $s_{i j} \rightarrow 0$, the integrals multiplying $\left|T_{i j, k \mid l, m}\right|^{2}$ have short-distance singularities and give rise to factors of $1 / s_{i j}$. Therefore, only permutations of $\left|T_{1,2,3 \mid 4,5}^{m}\right|^{2}$ and $\left|T_{12,3 \mid 4,5}\right|^{2} / s_{12}$ contribute at the lowest order $D^{2} \mathcal{R}^{5}$ in the low energy effective action [8]. This is the same order in the derivative expansion as the effective interaction $D^{4} \mathcal{R}^{4}$ appearing in the four-point genus-two amplitude at leading order, and indeed the couplings $D^{4} \mathcal{R}^{4}$ and $D^{2} \mathcal{R}^{5}$ are expected to belong to a single supersymmetric invariant under non-linear supersymmetry. As we shall see in section 3 , this is still the case for the genus-two $D^{4} \mathcal{R}^{5}$ and $D^{6} \mathcal{R}^{4}$ interactions, but the next order features a new five-point interaction $\left(D^{6} \mathcal{R}^{5}\right)^{\prime}$ which is no longer related to the corresponding four-point $D^{8} \mathcal{R}^{4}$ interaction. 


\section{The $\alpha^{\prime}$ expansion of genus-two integrals}

In this section, we shall decompose the integral for the amplitude with five external massless states, given in (2.49) for Type II strings, into a sum of basic integrals over $\Sigma^{5}$, in terms of which the full amplitude may be obtained by including suitable permutations of the external states. The low energy expansion of these integrals will be expressed in terms of genus-two MGFs, thereby generalizing a similar analysis carried out for the genus-two amplitude with four massless external states in [29-32]. These results will be used in section 4 to analyze the low energy expansion of the genus-two four-point and five-point amplitudes.

\subsection{Genus-two integrals occurring in Type II amplitudes}

In order to analyze the $\alpha^{\prime}$ expansion of the genus-two four-point and five-point amplitudes in Type II string theory, it will be useful to list the scalar integrals over four and five copies of the surface $\Sigma$ that occur along with the kinematic factors.

- The $I$-integrals occur in the four-point amplitude,

$$
\begin{aligned}
& I_{1}=\int_{\Sigma^{4}} \frac{\mathrm{KN}_{(4)}}{(\operatorname{det} Y)^{2}} \Delta(1,2) \Delta(3,4) \overline{\Delta(1,2)} \overline{\Delta(3,4)} \\
& I_{2}=\int_{\Sigma^{4}} \frac{\mathrm{KN}_{(4)}}{(\operatorname{det} Y)^{2}} \Delta(1,2) \Delta(3,4) \overline{\Delta(2,3)} \overline{\Delta(4,1)}
\end{aligned}
$$

- The $J$-integrals arise from the contributions $\left|\mathcal{V}_{I}^{m}\right|^{2}$ due to integrating a bilinear term in loop momenta (with cyclic identification $\omega_{I}(j+5)=\omega_{I}(j)$ ),

$$
\begin{aligned}
J_{r, s}= & \frac{i}{2} \int_{\Sigma^{5}} \frac{\mathrm{KN}_{(5)}}{(\operatorname{det} Y)^{2}} \omega_{I}(r) \Delta(r+1, r+2) \Delta(r+3, r+4) \\
& \times Y^{I J} \bar{\omega}_{J}(s) \overline{\Delta(s+1, s+2)} \overline{\Delta(s+3, s+4)}
\end{aligned}
$$

They may all be obtained by cyclic permutations from one of the three basic integrals,

$$
\begin{aligned}
J_{1,1} & =\frac{i}{2} \int_{\Sigma^{5}} \frac{\mathrm{KN}_{(5)}}{(\operatorname{det} Y)^{2}} \omega_{I}(1) Y^{I J} \bar{\omega}_{J}(1) \Delta(2,3) \Delta(4,5) \overline{\Delta(2,3)} \overline{\Delta(4,5)} \\
J_{1,2} & =\frac{i}{2} \int_{\Sigma^{5}} \frac{\mathrm{KN}_{(5)}}{(\operatorname{det} Y)^{2}} \omega_{I}(1) Y^{I J} \bar{\omega}_{J}(2) \Delta(2,3) \Delta(4,5) \overline{\Delta(3,4)} \overline{\Delta(5,1)} \\
J_{1,3} & =\frac{i}{2} \int_{\Sigma^{5}} \frac{\mathrm{KN}_{(5)}}{(\operatorname{det} Y)^{2}} \omega_{I}(1) Y^{I J} \bar{\omega}_{J}(3) \Delta(2,3) \Delta(4,5) \overline{\Delta(4,5)} \overline{\Delta(1,2)}
\end{aligned}
$$

and their complex conjugates.

- The $F$-integrals involve combinations of $\mathcal{G}(1,2) \mathcal{G}(1,2)$,

$$
\begin{aligned}
& F_{1}=\frac{1}{i \pi} \int_{\Sigma^{5}} \frac{\mathrm{KN}_{(5)}}{(\operatorname{det} Y)^{2}} \partial_{1} \mathcal{G}(1,2) \bar{\partial}_{1} \mathcal{G}(1,2) \Delta(2,3) \Delta(4,5) \overline{\Delta(2,3)} \overline{\Delta(4,5)} \\
& F_{2}=\frac{1}{i \pi} \int_{\Sigma^{5}} \frac{\mathrm{KN}_{(5)}}{(\operatorname{det} Y)^{2}} \partial_{1} \mathcal{G}(1,2) \bar{\partial}_{1} \mathcal{G}(1,2) \Delta(2,3) \Delta(4,5) \overline{\Delta(2,4)} \overline{\Delta(3,5)} \\
& F_{3}=\frac{1}{i \pi} \int_{\Sigma^{5}} \frac{\mathrm{KN}_{(5)}}{(\operatorname{det} Y)^{2}} \partial_{1} \mathcal{G}(1,2) \bar{\partial}_{2} \mathcal{G}(1,2) \Delta(2,3) \Delta(4,5) \overline{\Delta(1,3)} \overline{\Delta(4,5)} \\
& F_{4}=\frac{1}{i \pi} \int_{\Sigma^{5}} \frac{\mathrm{KN}_{(5)}}{(\operatorname{det} Y)^{2}} \partial_{1} \mathcal{G}(1,2) \bar{\partial}_{2} \mathcal{G}(1,2) \Delta(2,3) \Delta(4,5) \overline{\Delta(1,4)} \overline{\Delta(3,5)}
\end{aligned}
$$


- The $G$-integrals involve combinations of $\mathcal{G}(1,2) \mathcal{G}(1,3)$,

$$
\begin{aligned}
G_{1} & =\frac{1}{i \pi} \int_{\Sigma^{5}} \frac{\mathrm{KN}_{(5)}}{(\operatorname{det} Y)^{2}} \partial_{1} \mathcal{G}(1,2) \bar{\partial}_{1} \mathcal{G}(1,3) \Delta(2,4) \Delta(3,5) \overline{\Delta(2,4)} \overline{\Delta(3,5)} \\
G_{2} & =\frac{1}{i \pi} \int_{\Sigma^{5}} \frac{\mathrm{KN}_{(5)}}{(\operatorname{det} Y)^{2}} \partial_{1} \mathcal{G}(1,2) \bar{\partial}_{1} \mathcal{G}(1,3) \Delta(2,4) \Delta(3,5) \overline{\Delta(2,5)} \overline{\Delta(3,4)} \\
G_{3} & =\frac{1}{i \pi} \int_{\Sigma^{5}} \frac{\mathrm{KN}_{(5)}}{(\operatorname{det} Y)^{2}} \partial_{1} \mathcal{G}(1,2) \bar{\partial}_{3} \mathcal{G}(1,3) \Delta(2,4) \Delta(3,5) \overline{\Delta(2,4)} \overline{\Delta(1,5)} \\
G_{4} & =\frac{1}{i \pi} \int_{\Sigma^{5}} \frac{\mathrm{KN}_{(5)}}{(\operatorname{det} Y)^{2}} \partial_{1} \mathcal{G}(1,2) \bar{\partial}_{3} \mathcal{G}(1,3) \Delta(2,4) \Delta(3,5) \overline{\Delta(1,4)} \overline{\Delta(2,5)} \\
G_{5} & =\frac{1}{i \pi} \int_{\Sigma^{5}} \frac{\mathrm{KN}_{(5)}}{(\operatorname{det} Y)^{2}} \partial_{2} \mathcal{G}(1,2) \bar{\partial}_{3} \mathcal{G}(1,3) \Delta(1,4) \Delta(3,5) \overline{\Delta(1,4)} \overline{\Delta(2,5)} \\
G_{6} & =\frac{1}{i \pi} \int_{\Sigma^{5}} \frac{\mathrm{KN}_{(5)}}{(\operatorname{det} Y)^{2}} \partial_{2} \mathcal{G}(1,2) \bar{\partial}_{3} \mathcal{G}(1,3) \Delta(1,4) \Delta(3,5) \overline{\Delta(1,5)} \overline{\Delta(2,4)}
\end{aligned}
$$

- The $H$-integrals involve combinations of $\mathcal{G}(1,2) \mathcal{G}(3,4)$,

$$
\begin{aligned}
& H_{1}=\frac{1}{i \pi} \int_{\Sigma^{5}} \frac{\mathrm{KN}_{(5)}}{(\operatorname{det} Y)^{2}} \partial_{1} \mathcal{G}(1,2) \overline{\partial_{3} \mathcal{G}(3,4)} \Delta(2,4) \Delta(3,5) \overline{\Delta(2,4)} \overline{\Delta(1,5)} \\
& H_{2}=\frac{1}{i \pi} \int_{\Sigma^{5}} \frac{\mathrm{KN}_{(5)}}{(\operatorname{det} Y)^{2}} \partial_{1} \mathcal{G}(1,2) \overline{\partial_{3} \mathcal{G}(3,4)} \Delta(2,3) \Delta(4,5) \overline{\Delta(1,4)} \overline{\Delta(2,5)} \\
& H_{3}=\frac{1}{i \pi} \int_{\Sigma^{5}} \frac{\mathrm{KN}_{(5)}}{(\operatorname{det} Y)^{2}} \partial_{1} \mathcal{G}(1,2) \overline{\partial_{3} \mathcal{G}(3,4)} \Delta(2,3) \Delta(4,5) \overline{\Delta(1,5)} \overline{\Delta(2,4)} \\
& H_{4}=\frac{1}{i \pi} \int_{\Sigma^{5}} \frac{\mathrm{KN}_{(5)}}{(\operatorname{det} Y)^{2}} \partial_{1} \mathcal{G}(1,2) \overline{\partial_{3} \mathcal{G}(3,4)} \Delta(2,4) \Delta(3,5) \overline{\Delta(1,4)} \overline{\Delta(2,5)}
\end{aligned}
$$

All integrals required in the genus-two amplitude with five massless external states may be expressed in terms of the above integrals and permutations of their vertex labels. ${ }^{10}$

\subsection{Extracting the singular part of the $F$-integrals at $s_{i j}=0$}

All the integrals given in subsection 3.1 are absolutely convergent for $\left|s_{i j}\right| \ll 1$ and admit convergent Taylor series expansions at $s_{i j}=0$, with the notable exception of the $F$-integrals (3.4) which have simple poles at $s_{i j}=0$. In this subsection, we present the analysis needed to extract this singularity for the integral $F_{1}$ and defer the cases of the integrals $F_{2}, F_{3}, F_{4}$ to appendix B.4. The singularity of $F_{1}$ is due to the non-integrable singularity at $z_{1}=z_{2}$ of the following factor of the integrand of $F_{1}$,

$$
\partial_{1} \mathcal{G}(1,2) \bar{\partial}_{1} \mathcal{G}(1,2) \sim \frac{1}{\left|z_{1}-z_{2}\right|^{2}}
$$

\footnotetext{
${ }^{10}$ Note that it is convenient to explicitly add the complex conjugates $\bar{G}_{3}$ and $\bar{G}_{4}$ to the list above in order to quickly identify all the integrals in the genus-two correlator (2.49).
} 
As a result, the integral $F_{1}$ has a simple pole at $s_{12}=0$. The simple pole in $F_{1}$ may be exposed by using the following identity of the integrand,

$$
\begin{aligned}
\mathrm{KN}_{(5)} \partial_{1} \mathcal{G}(1,2) \bar{\partial}_{1} \mathcal{G}(1,2)= & -\frac{\mathrm{KN}_{(5)}}{s_{12}}\left(\sum_{k=3}^{5} s_{1 k} \partial_{1} \mathcal{G}(1,2) \bar{\partial}_{1} \mathcal{G}(1, k)+\partial_{1} \bar{\partial}_{1} \mathcal{G}(1,2)\right) \\
& +\frac{1}{s_{12}} \bar{\partial}_{1}\left(\mathrm{KN}_{(5)} \partial_{1} \mathcal{G}(1,2)\right)
\end{aligned}
$$

Since the combination inside the parentheses on the second line is a $(1,0)$ form its Dolbeault differential $\bar{\partial}_{1}$ may be recast as a total differential, $\bar{\partial}_{1}\left(\mathrm{KN}_{(5)} \partial_{1} \mathcal{G}(1,2)\right)=d_{1}\left(\mathrm{KN}_{(5)} \partial_{1} \mathcal{G}(1,2)\right)$ whose integral over the closed compact surface $\Sigma$ vanishes. As a result, the term on the second line does not contribute to $F_{1}$.

The contribution to the second term in the parentheses on the first line of (3.8) is given by (A.14). The $\delta(1,2)$ term vanishes provided we assume the following domain for $s_{12}$,

$$
\operatorname{Re}\left(s_{12}\right)<0
$$

or alternatively vanishes by the "cancelled propagator" argument in old string theory lingo. The remaining contributions to the integral $F_{1}$ are therefore given by the first term of the first line in (3.8) and by the $\kappa$-term in (A.14) for the second term in the parentheses, and we obtain the following formula,

$$
\begin{aligned}
F_{1}= & -\frac{1}{i \pi} \sum_{k=3}^{5} \frac{s_{1 k}}{s_{12}} \int_{\Sigma^{5}} \frac{\mathrm{KN}_{(5)}}{(\operatorname{det} Y)^{2}} \partial_{1} \mathcal{G}(1,2) \bar{\partial}_{1} \mathcal{G}(1, k)|\Delta(2,3)|^{2}|\Delta(4,5)|^{2} \\
& +\frac{2}{s_{12}} \int_{\Sigma^{5}} \frac{\mathrm{KN}_{(5)}}{(\operatorname{det} Y)^{2}} \kappa(1)|\Delta(2,3)|^{2}|\Delta(4,5)|^{2}
\end{aligned}
$$

The integrals in (3.10) are now absolutely convergent for $\left|s_{i j}\right| \ll 1$ and admit a convergent Taylor series expansion at $s_{i j}=0$. The coefficient of $1 / s_{12}$ is recognized as the integral $J_{1,1}$, and one can similarly express the first line of (3.10) in terms of permutations of the integrals $G_{1}, G_{2}$ defined in (3.5), see (B.32).

\subsection{Genus-two modular graph functions up to order $D^{6} \mathcal{R}^{5}$}

Our aim in this section will be to find the first few terms in the $\alpha^{\prime}$ expansions for the above integrals, so as to extract the coefficient of the effective interactions up to order $D^{6} \mathcal{R}^{5}$ in the low energy effective action. In addition to $\Delta(x, y)$, the following combination of holomorphic $(1,0)$-forms $\omega_{I}$ and their complex conjugates will be ubiquitous in our analysis, and are given as follows, in components,

$$
\nu(x, y)=\frac{i}{2} Y^{I J} \omega_{I}(x) \bar{\omega}_{J}(y)=-\overline{\nu(y, x)}
$$

On the diagonal $y=x$ they reduce to twice the canonical form $\kappa$ defined by,

$$
\kappa(x)=\frac{1}{2} \nu(x, x)=\frac{i}{4} Y^{I J} \omega_{I}(x) \bar{\omega}_{J}(x), \quad \int_{\Sigma} \kappa=1
$$

Various details of the subsequent computations are relegated to appendix B. 


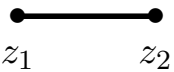

$\varphi$

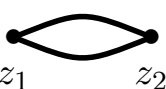

$\mathcal{Z}_{1}, \mathcal{Z}_{4}$

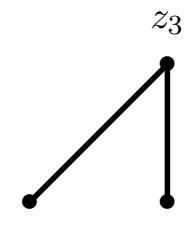

$z_{1}$

$\mathcal{Z}_{2}$

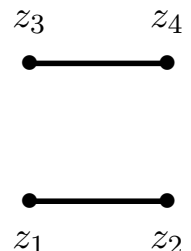

$\varphi^{2}, \mathcal{Z}_{3}$

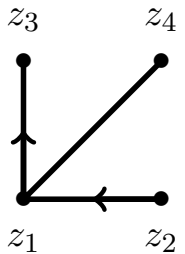

$\mathcal{Z}_{5}$

Figure 1. Graphs representing the genus-two modular graph functions $\varphi, \mathcal{Z}_{i}$ and $\varphi^{2}$ where each line represents the Green function $\mathcal{G}$ but the structure of the Abelian differentials is not exhibited. The arrows on two of the lines in $\mathcal{Z}_{5}$ indicate the derivatives $\partial_{1}$ and $\bar{\partial}_{1}$ on $\mathcal{G}$.

Up to order $D^{6} \mathcal{R}^{5}$, we find that the coefficients can all be expressed in terms of the Kawazumi-Zhang invariant $\varphi$ given by any one of the following equivalent expressions (see [29, 67] and references therein),

$$
\begin{aligned}
\varphi & =\int_{\Sigma^{2}} \frac{|\Delta(1,2)|^{2}}{4(\operatorname{det} Y)} \mathcal{G}(1,2) \\
& =-\frac{1}{4} Y^{I L} Y^{J K} \int_{\Sigma^{2}} \omega_{I}(1) \bar{\omega}_{J}(1) \omega_{K}(2) \bar{\omega}_{L}(2) \mathcal{G}(1,2) \\
& =\int_{\Sigma^{2}} \nu(1,2) \nu(2,1) \mathcal{G}(1,2)
\end{aligned}
$$

and the following convergent integrals on direct products of $\Sigma$,

$$
\begin{aligned}
& \mathcal{Z}_{1}=8 \int_{\Sigma^{2}} \kappa(1) \kappa(2) \mathcal{G}(1,2)^{2} \\
& \mathcal{Z}_{2}=-\int_{\Sigma^{3}} \frac{|\Delta(1,2)|^{2}}{\operatorname{det} Y} \kappa(3) \mathcal{G}(1,3) \mathcal{G}(2,3) \\
& \mathcal{Z}_{3}=\int_{\Sigma^{4}} \frac{|\Delta(1,3) \Delta(2,4)|^{2}}{8(\operatorname{det} Y)^{2}} \mathcal{G}(1,2) \mathcal{G}(3,4) \\
& \mathcal{Z}_{4}=-4 \int_{\Sigma^{2}} \nu(1,2) \nu(2,1) \mathcal{G}(1,2)^{2} \\
& \mathcal{Z}_{5}=\frac{16 i}{\pi} \int_{\Sigma^{4}} \mathcal{G}(1,4) \partial_{1} \mathcal{G}(1,2) \bar{\partial}_{1} \mathcal{G}(1,3) \nu(2,4) \nu(4,3) \nu(3,2)
\end{aligned}
$$

As is the case for the KZ invariant (3.13), the integrals (3.14) are modular invariant functions of the period matrix $\Omega$, and real analytic away from the separating and non-separating divisors. They belong to the class of genus-two MGFs introduced in [31], generalizing the genus-one MGFs of [33]. The relevant graphs keep track of the products of Arakelov Green functions to be integrated, and are displayed in figure 1.

The integrals $\mathcal{Z}_{1}, \mathcal{Z}_{2}, \mathcal{Z}_{3}$ have appeared previously in the study of the 4-point amplitude [31, 32], where their asymptotic behavior near the separating and non-separating divisors in the moduli space $\mathcal{M}_{2}$ was investigated in great detail. The integral $\mathcal{Z}_{4}$ was introduced in the course of the analysis of the action of the Laplace-Beltrami operator in [41], along with several other integrals which also occur here in the evaluation of the 
five-point amplitude, and which we evaluate in terms of the ones above in appendix A.6. The integral $\mathcal{Z}_{5}$ is novel, and reminiscent of the modular graph forms introduced in [43], although it is genuinely modular invariant. The asymptotics of $\mathcal{Z}_{4}$ and $\mathcal{Z}_{5}$ near the separating and non-separating divisors is derived in appendix $\mathrm{C}$ using similar methods as in [31, 32]. Genus-two amplitudes for the Heterotic string are expected to involve higher weight generalizations of these MGFs, in parallel with the modular graph forms appearing in Heterotic genus-one amplitudes [68].

\subsection{Novel modular graph function identities}

The study of the degenerations of the integrals $\mathcal{Z}_{i}$ in appendix $\mathrm{C}$ suggests that these integrals are not linearly independent, but rather satisfy a remarkable identity,

$$
\mathcal{Z}_{1}+\mathcal{Z}_{2}+\mathcal{Z}_{3}+\frac{1}{2} \mathcal{Z}_{4}-\varphi^{2}=0
$$

which we shall prove in appendix D. From the point of view of two-dimensional quantum field theory on the genus-two surface, the identity (3.15) is quite striking since it relates a combination of one-loop graphs $\mathcal{Z}_{1}, \mathcal{Z}_{4}$ to a combination of tree-level graphs $\varphi^{2}, \mathcal{Z}_{2}, \mathcal{Z}_{3}$. By contrast, the alternative expressions for $\varphi^{2}, \mathcal{Z}_{2}, \mathcal{Z}_{3}$ given in appendix A.6 exclusively relate tree-level graphs to one another and are the result of elementary relations, such as (A.7) and (A.8), between Abelian differentials. Thus, the identity (3.15) is more akin to the identities between genus-one MGFs proven in [42-45] and exposed by their representations in terms of iterated Eisenstein integrals [69, 70]. The proof of $(3.15)$, detailed in appendix D, makes crucial use of a lemma (D.1) valid at any genus $h$, which allows us, effectively, to convert a derivative $\partial_{i} \mathcal{G}\left(z_{i}, z_{j}\right)$ into a derivative $-\partial_{j} \mathcal{G}\left(z_{i}, z_{j}\right)$, despite the lack of translational invariance when $h \geq 2$. We anticipate that this property will become important in future studies of relations between genus-two MGFs. ${ }^{11}$

As a consequence of the genus-two identity (3.15) in the minimal non-separating degeneration limit $t \rightarrow \infty$ (with $t=\frac{\operatorname{det} \operatorname{Im} \Omega}{\operatorname{Im} \Omega_{11}}[31]$ ), we also obtain a new identity at genus one,

$$
\Delta_{\tau}\left(F_{2}^{2}-2 F_{4}\right)=6 F_{2}^{2}-4 F_{4}
$$

where $F_{k}(v \mid \tau)$ is the elliptic MGF on a torus $\Sigma_{1}$ of modulus $\tau$ with $v \in \Sigma_{1}$, defined by,

$$
F_{k}(v \mid \tau)=\frac{1}{k !} \int_{\Sigma_{1}} \kappa_{1}(x) f(x)^{k} \quad \kappa_{1}(x)=\frac{i}{2 \tau_{2}} d x d \bar{x}
$$

Here $f(x)=g\left(x-p_{b}\right)-g\left(x-p_{a}\right)$ where $g$ is the Green function $g$ on $\Sigma_{1}$ and $p_{a}, p_{b}$ are two punctures on $\Sigma_{1}$ with $v=p_{b}-p_{a}$ (see appendix C). The Laplacian on $\tau$, defined by $\Delta_{\tau}=4 \tau_{2}^{2} \partial_{\tau} \partial_{\bar{\tau}}$, acts on elliptic functions of $v=u_{1}+u_{2} \tau$ by keeping the real coordinates $u_{1}, u_{2}$ fixed. Identity (3.16) is again reminiscent of the identities proven in [42-45]. ${ }^{12}$

\footnotetext{
${ }^{11}$ Indeed, the conversion of derivatives has been used to generalize (3.15) to arbitrary genus and to derive higher-weight identities [71] since the first version of this work.

${ }^{12}$ In an earlier version of this work, it was left as an open problem to derive (3.16) directly, without recourse to its genus-two ancestor. Since then, a direct proof has been given in [72] using genus-one methods.
} 


\subsection{Expansion in $\alpha^{\prime}$ of the basic genus-two integrals}

In this subsection, we shall list the results of the expansions of the integrals $J_{1, i}$ to order $s_{i j}^{2}$ and of $F_{j}, G_{j}, H_{j}$ to order $s_{i j}$. Their derivations are relegated to appendix B. We also include the expansion for the integrals $I_{1}, I_{2}$ governing the four-point amplitude [32].

- For the four-point integrals in (3.1),

$$
\begin{aligned}
I_{1}= & 64-64 s_{12} \varphi+\left(24 s_{12}^{2}-16 s_{13} s_{23}\right)\left(\mathcal{Z}_{1}-2 \mathcal{Z}_{2}+\mathcal{Z}_{3}\right) \\
& +s_{12}^{2}\left(48 \mathcal{Z}_{2}+8 \mathcal{Z}_{4}-16 \mathcal{Z}_{3}+16 \varphi^{2}\right)+\mathcal{O}\left(s_{i j}^{3}\right) \\
I_{2}= & 32+64 \varphi s_{13}+8\left(s_{12}^{2}+s_{23}^{2}\right)\left(\mathcal{Z}_{1}-2 \mathcal{Z}_{2}+\mathcal{Z}_{3}\right) \\
& -s_{12} s_{23}\left(48 \mathcal{Z}_{2}+8 \mathcal{Z}_{4}-16 \mathcal{Z}_{3}+16 \varphi^{2}\right)+\mathcal{O}\left(s_{i j}^{3}\right)
\end{aligned}
$$

- For the five-point $J$-integrals in (3.3),

$$
\begin{aligned}
J_{1,1}= & 128-64\left(s_{23}+s_{45}\right) \varphi+16 s_{23} s_{45}\left(-2 \mathcal{Z}_{1}+\mathcal{Z}_{3}+2 \varphi^{2}\right)+8\left(s_{23}^{2}+s_{45}^{2}\right)\left(\mathcal{Z}_{4}+5 \mathcal{Z}_{1}\right) \\
& +16\left(s_{12} s_{15}+2 s_{34}^{2}-2 s_{12} s_{34}-2 s_{15} s_{34}+s_{23} s_{34}+s_{34} s_{45}\right)\left(\mathcal{Z}_{1}-2 \mathcal{Z}_{2}+\mathcal{Z}_{3}\right) \\
& +32\left(s_{12}^{2}+s_{15}^{2}-s_{15} s_{23}-s_{45} s_{12}\right)\left(\mathcal{Z}_{1}-\mathcal{Z}_{2}\right)+16\left(s_{12} s_{23}+s_{45} s_{15}\right)\left(\mathcal{Z}_{1}-\mathcal{Z}_{3}\right)+\mathcal{O}\left(s_{i j}^{3}\right) \\
J_{1,2}= & 32+64 s_{35} \varphi+8\left(s_{12}^{2}+s_{34}^{2}+s_{45}^{2}\right)\left(\mathcal{Z}_{1}-2 \mathcal{Z}_{2}+\mathcal{Z}_{3}\right)+8 s_{15} s_{23}\left(2 \mathcal{Z}_{2}+3 \mathcal{Z}_{3}+\mathcal{Z}_{4}\right) \\
& -8 s_{12}\left(s_{34}+s_{45}\right)\left(\mathcal{Z}_{1}-4 \mathcal{Z}_{2}+3 \mathcal{Z}_{3}\right)+8 s_{12}\left(s_{15}+s_{23}\right)\left(\mathcal{Z}_{1}+\mathcal{Z}_{2}+\mathcal{Z}_{3}+\frac{1}{2} \mathcal{Z}_{4}-\varphi^{2}\right) \\
& -4 s_{34} s_{45}\left(10 \mathcal{Z}_{2}-10 \mathcal{Z}_{3}+\mathcal{Z}_{4}+10 \varphi^{2}\right)-4\left(s_{23} s_{34}+s_{45} s_{15}\right)\left(2 \mathcal{Z}_{2}+6 \mathcal{Z}_{3}+\mathcal{Z}_{4}-6 \varphi^{2}\right) \\
& +8\left(s_{15}^{2}+s_{23}^{2}-s_{23} s_{45}-s_{34} s_{15}\right)\left(\mathcal{Z}_{1}-\mathcal{Z}_{3}\right)+\mathcal{O}\left(s_{i j}^{3}\right) \\
\left.J_{1,3}\right)= & -64+64\left(s_{45}-s_{13}\right) \varphi-16\left(s_{12}^{2}+s_{23}^{2}\right)\left(\mathcal{Z}_{1}-\mathcal{Z}_{2}\right)+8 s_{45}^{2}\left(-2 \mathcal{Z}_{1}+\mathcal{Z}_{3}+2 \varphi^{2}\right) \\
& +16\left(s_{15} s_{23}+s_{12} s_{34}-s_{34}^{2}-s_{15}^{2}\right)\left(\mathcal{Z}_{1}-2 \mathcal{Z}_{2}+\mathcal{Z}_{3}\right)+8 s_{12} s_{23}\left(2 \mathcal{Z}_{2}+3 \mathcal{Z}_{3}+\mathcal{Z}_{4}\right) \\
& +8\left(2 s_{15} s_{34}-s_{12} s_{15}-s_{23} s_{34}\right)\left(\mathcal{Z}_{1}-4 \mathcal{Z}_{2}+3 \mathcal{Z}_{3}\right)+8\left(s_{15}+s_{34}\right) s_{45}\left(\mathcal{Z}_{3}-\mathcal{Z}_{1}\right) \\
& +8\left(s_{12}+s_{23}\right) s_{45}\left(\mathcal{Z}_{1}-2 \mathcal{Z}_{2}-2 \mathcal{Z}_{3}-\mathcal{Z}_{4}-4 \varphi^{2}\right)+\mathcal{O}\left(s_{i j}^{3}\right)
\end{aligned}
$$

- For the F-integrals (3.4), with kinematic poles exposed via (3.10),

$$
\begin{aligned}
F_{1}= & \frac{128}{s_{12}}+\frac{64 \varphi\left(s_{12}-2 s_{45}\right)}{s_{12}}-\frac{32 s_{34} s_{35}}{s_{12}}\left(\mathcal{Z}_{1}-2 \mathcal{Z}_{2}+\mathcal{Z}_{3}\right)+\frac{16 s_{45}^{2}}{s_{12}}\left(3 \mathcal{Z}_{1}+\mathcal{Z}_{3}+\mathcal{Z}_{4}+2 \varphi^{2}\right) \\
& +8 s_{23}\left(-\mathcal{Z}_{1}-2 \mathcal{Z}_{2}-\mathcal{Z}_{4}\right)+32\left(\mathcal{Z}_{1}-\mathcal{Z}_{2}\right) s_{12} \\
& -8\left(5 \mathcal{Z}_{1}-6 \mathcal{Z}_{2}+2 \mathcal{Z}_{3}+\mathcal{Z}_{4}+4 \varphi^{2}\right) s_{45}+\mathcal{O}\left(s_{i j}^{2}\right) \\
F_{2}= & \frac{64}{s_{12}}+\frac{32 \varphi\left(4 s_{34}-s_{12}\right)}{s_{12}}+\frac{16 s_{34}^{2}}{s_{12}}\left(\mathcal{Z}_{1}-2 \mathcal{Z}_{2}+\mathcal{Z}_{3}\right)-\frac{16 s_{35} s_{45}}{s_{12}}\left(2 \mathcal{Z}_{1}+2 \mathcal{Z}_{2}+\mathcal{Z}_{4}+2 \varphi^{2}\right) \\
& +16 \mathcal{Z}_{1} s_{12}+8\left(-\mathcal{Z}_{1}-2 \mathcal{Z}_{2}-\mathcal{Z}_{4}\right) s_{15}-16\left(\mathcal{Z}_{1}+\mathcal{Z}_{3}\right) s_{34}+\mathcal{O}\left(s_{i j}^{2}\right) \\
F_{3}= & -\frac{128}{s_{12}}+\frac{128 \varphi\left(s_{45}-s_{12}\right)}{s_{12}}+\frac{32 s_{34} s_{35}}{s_{12}}\left(\mathcal{Z}_{1}-2 \mathcal{Z}_{2}+\mathcal{Z}_{3}\right)-\frac{16 s_{45}^{2}}{s_{12}}\left(3 \mathcal{Z}_{1}+\mathcal{Z}_{3}+\mathcal{Z}_{4}+2 \varphi^{2}\right) \\
& -32 s_{12}\left(\mathcal{Z}_{1}-\mathcal{Z}_{2}\right)+16 s_{45}\left(3 \mathcal{Z}_{1}-3 \mathcal{Z}_{2}+\mathcal{Z}_{3}+\mathcal{Z}_{4}+4 \varphi^{2}\right)+\mathcal{O}\left(s_{i j}^{2}\right) \\
F_{4}= & -\frac{64}{s_{12}}-\frac{128 \varphi s_{34}}{s_{12}}-\frac{16 s_{34}^{2}}{s_{12}}\left(\mathcal{Z}_{1}-2 \mathcal{Z}_{2}+\mathcal{Z}_{3}\right)+\frac{16 s_{35} s_{45}}{s_{12}}\left(2 \mathcal{Z}_{1}+2 \mathcal{Z}_{2}+\mathcal{Z}_{4}+2 \varphi^{2}\right) \\
& +8 s_{12}\left(-\mathcal{Z}_{2}-2 \mathcal{Z}_{1}+2 \varphi^{2}\right)+16 s_{34}\left(\mathcal{Z}_{1}+\mathcal{Z}_{2}+\mathcal{Z}_{3}-2 \varphi^{2}\right)+\mathcal{O}\left(s_{i j}^{2}\right)
\end{aligned}
$$


- For the $G$-integrals in (3.5),

$$
\begin{aligned}
G_{1}= & -16 s_{23} \mathcal{Z}_{1}-16\left(s_{25}+s_{34}\right) \mathcal{Z}_{2}-16 s_{45} \mathcal{Z}_{3}+\mathcal{O}\left(s_{i j}^{2}\right) \\
G_{2}= & -32 \varphi+\left(s_{14}-s_{15}\right) \mathcal{Z}_{5}+4\left(s_{12}+s_{13}\right)\left(\mathcal{Z}_{1}+\mathcal{Z}_{4}\right)+8 s_{23} \mathcal{Z}_{4} \\
& -8 \mathcal{Z}_{2}\left(s_{24}+s_{25}+s_{34}+s_{35}\right)+16 s_{45}\left(-\mathcal{Z}_{3}+\varphi^{2}\right)+\mathcal{O}\left(s_{i j}^{2}\right) \\
G_{3}= & 16 s_{23}\left(\mathcal{Z}_{1}+\mathcal{Z}_{2}\right)+16 s_{34}\left(\mathcal{Z}_{2}+\mathcal{Z}_{3}\right)+\mathcal{O}\left(s_{i j}^{2}\right) \\
G_{4}= & 8 s_{23}\left(\mathcal{Z}_{2}-\mathcal{Z}_{4}\right)+8 s_{35}\left(\mathcal{Z}_{2}+2 \varphi^{2}\right)+8 s_{34}\left(\mathcal{Z}_{2}+2 \mathcal{Z}_{3}-2 \varphi^{2}\right)+\mathcal{O}\left(s_{i j}^{2}\right) \\
G_{5}= & -16 s_{23}\left(\mathcal{Z}_{1}+\mathcal{Z}_{2}\right)+\mathcal{O}\left(s_{i j}^{2}\right) \\
G_{6}= & 8 s_{23}\left(\mathcal{Z}_{4}-\mathcal{Z}_{2}\right)+\mathcal{O}\left(s_{i j}^{2}\right)
\end{aligned}
$$

- For the $H$ - integrals in (3.6),

$$
\begin{aligned}
& H_{1}=-16 s_{13}\left(\mathcal{Z}_{2}+\mathcal{Z}_{3}\right)+\mathcal{O}\left(s_{i j}^{2}\right) \\
& H_{2}=-8 s_{13}\left(\mathcal{Z}_{2}+2 \mathcal{Z}_{3}-2 \varphi^{2}\right)+\mathcal{O}\left(s_{i j}^{2}\right) \\
& H_{3}=-8 s_{13}\left(\mathcal{Z}_{2}+2 \mathcal{Z}_{3}-2 \varphi^{2}\right)+\mathcal{O}\left(s_{i j}^{2}\right) \\
& H_{4}=-8 s_{13}\left(\mathcal{Z}_{2}+2 \mathcal{Z}_{3}-2 \varphi^{2}\right)+\mathcal{O}\left(s_{i j}^{2}\right)
\end{aligned}
$$

\subsection{Decomposing the five-point correlator}

The five-point integrand (2.49) is expressible via permutations and complex conjugation of the integrals discussed above and will be decomposed into four sectors according to the appearance and arguments of the Arakelov Green functions $\mathcal{G}$,

$$
\mathcal{B}_{(5)}=\mathcal{B}_{(5)}^{J}+\mathcal{B}_{(5)}^{F}+\mathcal{B}_{(5)}^{G}+\mathcal{B}_{(5)}^{H}
$$

where the superscripts indicate the type of integral involved in the decomposition. The first part comprises the contractions of the vector blocks between the left- and right-movers, resulting from integrating out the loop momenta, ${ }^{13}$

$$
\begin{aligned}
\mathcal{B}_{(5)}^{J}= & i \int_{\Sigma^{5}} \frac{\mathrm{KN}_{(5)}}{(\operatorname{det} Y)^{2}} Y^{I J} \mathcal{V}_{I}^{m} \tilde{\mathcal{V}}_{J}^{m} \\
= & 2\left\{J_{1,1} T_{5,1,2 \mid 3,4}^{m} \tilde{T}_{5,1,2 \mid 3,4}^{m}+J_{1,2} T_{5,1,2 \mid 3,4}^{m} \tilde{T}_{1,2,3 \mid 4,5}^{m}+\overline{J_{1,2}} T_{1,2,3 \mid 4,5}^{m} \tilde{T}_{5,1,2 \mid 3,4}^{m}\right. \\
& \left.+J_{1,3} T_{5,1,2 \mid 3,4}^{m} \tilde{T}_{2,3,4 \mid 5,1}^{m}+\overline{J_{1,3}} T_{2,3,4 \mid 5,1}^{m} \tilde{T}_{5,1,2 \mid 3,4}^{m}+\operatorname{cycl}(1,2,3,4,5)\right\}
\end{aligned}
$$

The remaining three parts, $\mathcal{B}_{(5)}^{F}, \mathcal{B}_{(5)}^{G}$ and $\mathcal{B}_{(5)}^{H}$ are organized by the number of labels shared between the scalar building blocks $\mathcal{Q}_{a b}$ and $\tilde{\mathcal{Q}}_{c d}$ defined in (2.11) and the positions of the derivatives on the Arakelov Green functions. It will be convenient to express these contributions as sums over permutations of more elementary building blocks,

$$
\begin{aligned}
\mathcal{B}_{(5)}^{F} & =\mathcal{B}_{12}^{F}+(1,2 \mid 1,2,3,4,5) \\
\mathcal{B}_{(5)}^{G} & =\left[\mathcal{B}_{1 ; 23}^{G}+\mathcal{B}_{1 ; 32}^{G}+(2,3 \mid 2,3,4,5)\right]+(1 \leftrightarrow 2,3,4,5) \\
\mathcal{B}_{(5)}^{H} & =\left[\mathcal{B}_{12,34}^{H}+\mathcal{B}_{34,12}^{H}+\operatorname{cyc}(2,3,4)\right]+(5 \leftrightarrow 1,2,3,4)
\end{aligned}
$$

\footnotetext{
${ }^{13}$ Throughout, complex conjugation on an integral will leave the kinematic variables $s_{i j}$ unchanged.
} 
where the combinations of the type $\mathcal{G}(1,2) \mathcal{G}(1,2)$ yield, ${ }^{14}$

$$
\begin{aligned}
\mathcal{B}_{12}^{F}=\int_{\Sigma^{5}} \frac{\mathcal{Q}_{12} \tilde{\mathcal{Q}}_{12} \mathrm{KN}_{(5)}}{i \pi(\operatorname{det} Y)^{2}}= & S_{1 ; 2|3| 4,5} \tilde{S}_{1 ; 2|3| 4,5}\left(\left.F_{1}\right|_{3 \leftrightarrow 4}\right)+S_{1 ; 2|3| 4,5} \tilde{S}_{1 ; 2|4| 3,5}\left(\left.F_{2}\right|_{3 \leftrightarrow 4}\right) \\
& +S_{1 ; 2|4| 3,5} \tilde{S}_{1 ; 2|3| 4,5} F_{2}+S_{1 ; 2|4| 3,5} \tilde{S}_{1 ; 2|4| 3,5} F_{1} \\
& +S_{2 ; 1|3| 4,5} \tilde{S}_{2 ; 1|3| 4,5}\left(\left.F_{1}\right|_{3 \leftrightarrow 4} ^{1 \leftrightarrow 2}\right)+S_{2 ; 1|3| 4,5} \tilde{S}_{2 ; 1|4| 3,5}\left(\left.F_{2}\right|_{3 \leftrightarrow 4} ^{1 \leftrightarrow 2}\right) \\
& +S_{2 ; 1|4| 3,5} \tilde{S}_{2 ; 1|3| 4,5}\left(\left.F_{2}\right|^{1 \leftrightarrow 2}\right)+S_{2 ; 1|4| 3,5} \tilde{S}_{2 ; 1|4| 3,5}\left(\left.F_{1}\right|^{1 \leftrightarrow 2}\right) \\
& +S_{1 ; 2|3| 4,5} \tilde{S}_{2 ; 1|3| 4,5}\left(\left.F_{3}\right|_{3 \leftrightarrow 4}\right)+S_{1 ; 2|3| 4,5} \tilde{S}_{2 ; 1|4| 3,5}\left(\left.F_{4}\right|_{3 \leftrightarrow 4}\right) \\
& +S_{1 ; 2|4| 3,5} \tilde{S}_{2 ; 1|3| 4,5} F_{4}+S_{1 ; 2|4| 3,5} \tilde{S}_{2 ; 1|4| 3,5} F_{3} \\
& +S_{2 ; 1|3| 4,5} \tilde{S}_{1 ; 2|3| 4,5}\left(\left.F_{3}\right|_{3 \leftrightarrow 4} ^{1 \leftrightarrow 2}\right)+S_{2 ; 1|3| 4,5} \tilde{S}_{1 ; 2|4| 3,5}\left(\left.F_{4}\right|_{3 \leftrightarrow 4} ^{1 \leftrightarrow 2}\right) \\
& +S_{2 ; 1|4| 3,5} \tilde{S}_{1 ; 2|3| 4,5}\left(\left.F_{4}\right|^{1 \leftrightarrow 2}\right)+S_{2 ; 1|4| 3,5} \tilde{S}_{1 ; 2|4| 3,5}\left(\left.F_{3}\right|^{1 \leftrightarrow 2}\right)
\end{aligned}
$$

the combinations of the type $\mathcal{G}(1,2) \mathcal{G}(1,3)$ yield,

$$
\begin{aligned}
\mathcal{B}_{1 ; 23}^{G}=\int_{\Sigma^{5}} \frac{\mathcal{Q}_{12} \tilde{\mathcal{Q}}_{13} \mathrm{KN}_{(5)}}{i \pi(\operatorname{det} Y)^{2}}= & S_{1 ; 2|4| 3,5} \tilde{S}_{1 ; 3|4| 2,5}\left(\left.G_{2}\right|_{4 \leftrightarrow 5}\right)+S_{1 ; 2|4| 3,5} \tilde{S}_{1 ; 3|5| 2,4}\left(\left.G_{1}\right|_{4 \leftrightarrow 5}\right) \\
& +S_{1 ; 2|5| 3,4} \tilde{S}_{1 ; 3|4| 2,5} G_{1}+S_{1 ; 2|5| 3,4} \tilde{S}_{1 ; 3|5| 2,4} G_{2} \\
& +S_{1 ; 2|4| 3,5} \tilde{S}_{3 ; 1|4| 2,5}\left(\left.G_{4}\right|_{4 \leftrightarrow 5}\right)+S_{1 ; 2|4| 3,5} \tilde{S}_{3 ;|| 5 \mid 2,4}\left(\left.G_{3}\right|_{4 \leftrightarrow 5}\right) \\
& +S_{1 ; 2|5| 3,4} \tilde{S}_{3 ; 1|4| 2,5} G_{3}+S_{1 ; 2|5| 3,4} \tilde{S}_{3 ; 1|5| 2,4} G_{4} \\
& +S_{2 ; 1|4| 3,5} \tilde{S}_{1 ; 3|4| 2,5}\left(\left.\bar{G}_{4}\right|_{4 \leftrightarrow 5} ^{2 \leftrightarrow 3}\right)+S_{2 ; 1|4| 3,5} \tilde{S}_{1 ; 3|5| 2,4}\left(\left.\bar{G}_{3}\right|^{2 \leftrightarrow 3}\right) \\
& +S_{2 ; 1|5| 3,4} \tilde{S}_{1 ; 3|4| 2,5}\left(\left.\bar{G}_{3}\right|_{4 \leftrightarrow 5} ^{2 \leftrightarrow 3}\right)+S_{2 ; 1|5| 3,4} \tilde{S}_{1 ; 3|5| 2,4}\left(\left.\bar{G}_{4}\right|^{2 \leftrightarrow 3}\right) \\
& +S_{2 ; 1|4| 3,5} \tilde{S}_{3 ; 1|4| 2,5}\left(\left.G_{5}\right|_{4 \leftrightarrow 5}\right)+S_{2 ; 1|4| 3,5} \tilde{S}_{3 ;|5| 2,4}\left(\left.G_{6}\right|_{4 \leftrightarrow 5}\right) \\
& +S_{2 ; 1|5| 3,4} \tilde{S}_{3 ; 1|4| 2,5} G_{6}+S_{2 ; 1|5| 3,4} \tilde{S}_{3 ; 1|5| 2,4} G_{5}
\end{aligned}
$$

and the combinations of the type $\mathcal{G}(1,2) \mathcal{G}(3,4)$ yield,

$$
\begin{aligned}
\mathcal{B}_{12,34}^{H}=\int_{\Sigma^{5}} \frac{\mathcal{Q}_{12} \tilde{\mathcal{Q}}_{34} \mathrm{KN}_{(5)}}{i \pi(\operatorname{det} Y)^{2}}= & -S_{1 ; 2|3| 4,5} \tilde{S}_{3 ; 4|1| 2,5} H_{1}-S_{1 ; 2|3| 4,5} \tilde{S}_{3 ; 4|2| 1,5} H_{4} \\
& -S_{1 ; 2|4| 3,5} \tilde{S}_{3 ; 4|1| 2,5} H_{3}-S_{1 ; 2|4| 3,5} \tilde{S}_{3 ; 4|2| 1,5} H_{2} \\
& -S_{2 ; 1|3| 4,5} \tilde{S}_{3 ; 4|1| 2,5}\left(\left.H_{4}\right|_{1 \leftrightarrow 2}\right)-S_{2 ; 1|3| 4,5} \tilde{S}_{3 ; 4|2| 1,5}\left(\left.H_{1}\right|_{1 \leftrightarrow 2}\right) \\
& -S_{2 ; 1|4| 3,5} \tilde{S}_{3 ; 4|1| 2,5}\left(\left.H_{2}\right|_{1 \leftrightarrow 2}\right)-S_{2 ; 1|4| 3,5} \tilde{S}_{3 ; 4|2| 1,5}\left(\left.H_{3}\right|_{1 \leftrightarrow 2}\right) \\
& -S_{1 ; 2|3| 4,5} \tilde{S}_{4 ; 3|1| 2,5}\left(\left.H_{3}\right|^{3 \leftrightarrow 4}\right)-S_{1 ; 2|3| 4,5} \tilde{S}_{4 ; 3|2| 1,5}\left(\left.H_{2}\right|^{3 \leftrightarrow 4}\right) \\
& -S_{1 ; 2|4| 3,5} \tilde{S}_{4 ; 3|1| 2,5}\left(\left.H_{1}\right|^{3 \leftrightarrow 4}\right)-S_{1 ; 2|4| 3,5} \tilde{S}_{4 ; 3|2| 1,5}\left(\left.H_{4}\right|^{3 \leftrightarrow 4}\right) \\
& -S_{2 ; 1|3| 4,5} \tilde{S}_{4 ; 3|1| 2,5}\left(\left.H_{2}\right|_{1 \leftrightarrow 4} ^{3 \leftrightarrow 4}\right)-S_{2 ; 1|3| 4,5} \tilde{S}_{4 ; 3|2| 1,5}\left(\left.H_{3}\right|_{1 \leftrightarrow 2} ^{3 \leftrightarrow 4}\right) \\
& -S_{2 ; 1|4| 3,5} \tilde{S}_{4 ; 3|1| 2,5}\left(\left.H_{4}\right|_{1 \leftrightarrow 4} ^{3 \leftrightarrow 4}\right)-S_{2 ; 1|4| 3,5} \tilde{S}_{4 ; 3|2| 1,5}\left(\left.H_{1}\right|_{1 \leftrightarrow 2} ^{3 \leftrightarrow 4}\right)
\end{aligned}
$$

\footnotetext{
${ }^{14}$ Throughout, a vertical bar with permutations of the vertex labels following an integral function will indicate the permutation to be performed on the entries of the integrals as they were originally defined in (3.3), (3.4), (3.5), (3.6).
} 
One can readily recast the expressions above in terms of manifestly BRST-invariant building blocks valid for all external states via $\left(S_{a ; b|c| d, e}, T_{a, b, c \mid d, e}^{m}\right) \rightarrow\left(s_{a b} C_{a ; b|c| d, e}, C_{a, b, c \mid d, e}^{m}\right)$. When truncating to the bosonic component sector one may use the effective building blocks of section 2.4 as $\left(S_{a ; b|c| d, e}, T_{a, b, c \mid d, e}^{m}\right) \rightarrow\left(S_{a ; b|c| d, e}^{\text {eff }}, T_{a, b, c \mid d, e}^{m, \text { eff }}\right)$.

\section{The $\alpha^{\prime}$ expansion of genus-two amplitudes}

In this section we shall combine the expansions of the integrals studied in section 3 in order to extract the low energy expansion of the genus-two five-point amplitude (2.45). As a warm-up, we first consider the low energy expansion of the genus-two four-point amplitude, studied in [29-32]. We will follow the normalization conventions based on the first-principles computations in the non-minimal pure spinor formalism $[8,57,73]$.

\subsection{The four-point amplitude}

The four-point amplitude at two loops in the pure spinor formalism is given by $[8,57]^{15}$

$$
\mathcal{A}_{(4)}^{\text {genus }-2}=\delta(k) \mathcal{N}_{(4)} \int_{\mathcal{M}_{2}} d \mu_{2} \mathcal{B}_{(4)}\left(k_{i} \mid \Omega\right)
$$

The normalization factor $\mathcal{N}_{(4)}$ is given by, ${ }^{16}$

$$
\mathcal{N}_{(4)}=\frac{\kappa^{4} e^{2 \lambda}}{2^{25} \pi^{6}}\left(\frac{\alpha^{\prime}}{2}\right)^{5}
$$

in terms of the normalization constant of the massless vertex operators $\kappa$ [28], and the bare expectation values of the dilaton $\phi$. The S-duality analysis of [8] relates,

$$
e^{2 \lambda}=2^{6} \pi^{4} e^{2 \phi}
$$

The integrand on $\mathcal{M}_{2}$ in turn is given by the integral $\mathcal{B}_{(4)}\left(k_{i} \mid \Omega\right)$ over the four vertex points defined in (2.47). With the expression (2.7) for the left chiral correlator $\mathcal{K}_{(4)}$, the integrand of (4.1) can be expressed straightforwardly in terms of the $I$-integrals defined in (3.1) as

$$
\begin{aligned}
\mathcal{B}_{(4)}\left(k_{i} \mid \Omega\right)= & I_{1}\left|T_{1,4 \mid 2,3}\right|^{2}+\left(\left.I_{1}\right|_{2 \leftrightarrow 4}\right)\left|T_{1,2 \mid 3,4}\right|^{2} \\
& +I_{2}\left(T_{1,2 \mid 3,4} \tilde{T}_{1,4 \mid 2,3}+T_{1,4 \mid 2,3} \tilde{T}_{1,2 \mid 3,4}\right)
\end{aligned}
$$

Using the symmetry property $T_{1,3 \mid 2,4}=-T_{1,4 \mid 2,3}-T_{1,2 \mid 3,4}$ of (2.15), momentum conservation, as well as the expansions (3.18) we obtain the following expansion for the integrand,

$$
\begin{aligned}
\mathcal{B}_{(4)}\left(k_{i} \mid \Omega\right)= & 32\left(\left|T_{1,2 \mid 3,4}\right|^{2}+\left|T_{1,3 \mid 2,4}\right|^{2}+\left|T_{1,4 \mid 2,3}\right|^{2}\right) \\
& +64 \varphi\left(s_{12}\left|T_{1,2 \mid 3,4}\right|^{2}+s_{13}\left|T_{1,3 \mid 2,4}\right|^{2}+s_{14}\left|T_{1,4 \mid 2,3}\right|^{2}\right) \\
& +8 A_{1}\left(s_{12}^{2}\left|T_{1,2 \mid 3,4}\right|^{2}+s_{13}^{2}\left|T_{1,3 \mid 2,4}\right|^{2}+s_{14}^{2}\left|T_{1,4 \mid 2,3}\right|^{2}\right) \\
& +16 A_{2}\left|s_{14} T_{1,2 \mid 3,4}-s_{12} T_{1,4 \mid 2,3}\right|^{2}+\cdots
\end{aligned}
$$

\footnotetext{
${ }^{15}$ In equation (4.1) we have absorbed an overall factor of $2^{20} 3^{6} 5^{2}$ coming from $\mathcal{B}_{(4)}\left(k_{i} \mid \Omega\right)$ into the normalization of the four-point amplitude given in [8].

${ }^{16}$ Alternatively, $\mathcal{N}_{(4)}$ may be presented in terms of the 10 -dimensional Newton constant $\kappa_{10}^{2}$ and the vacuum expectation value of the dilaton $\phi$, canonically normalized in Type IIB [28, 29], as follows $\mathcal{N}_{(4)}=$ $2^{-6} \pi \kappa_{10}^{2} e^{2 \phi}$.
} 
where the terms in the ellipsis feature $\mathcal{O}\left(s_{i j}^{3}\right)$ along with $\left|T_{a, b \mid c, d}\right|^{2}$, and we have defined the following combinations,

$$
\begin{aligned}
& A_{1}=\mathcal{Z}_{1}-2 \mathcal{Z}_{2}+\mathcal{Z}_{3} \\
& A_{2}=\varphi^{2}+\mathcal{Z}_{1}+\mathcal{Z}_{2}+\frac{1}{2} \mathcal{Z}_{4}=2 \varphi^{2}-\mathcal{Z}_{3}
\end{aligned}
$$

As shown in [74], the components $\left\langle T_{1,2 \mid 3,4}\right\rangle_{0}$ of the genus-two building block are given by,

$$
\left\langle T_{1,2 \mid 3,4}\right\rangle_{0}=16\left(\frac{2}{\alpha^{\prime}}\right) s_{12}\left\langle V_{1} T_{2,3,4}\right\rangle_{0}
$$

where $K=\left\langle V_{1} T_{2,3,4}\right\rangle_{0}$ is the usual one-loop kinematic factor, which is permutationsymmetric and reduces to the usual $t_{8} F^{4}=t_{8}\left(f_{1}, f_{2}, f_{3}, f_{4}\right)$ combination for external gauge fields. Substituting (4.7) into (4.5), the last line cancels and one arrives at,

$$
\mathcal{B}_{4}\left(k_{i} \mid \Omega\right)=2^{13} K \tilde{K}\left(\frac{2}{\alpha^{\prime}}\right)^{2}\left[\sigma_{2}+2 \varphi \sigma_{3}+\frac{1}{4} A_{1} \sigma_{4}+\mathcal{O}\left(s_{i j}^{5}\right)\right]
$$

where $\sigma_{k}$ are the usual symmetric polynomials in four-point kinematic variables,

$$
\sigma_{k}=s_{12}^{k}+s_{13}^{k}+s_{14}^{k}
$$

Substituting (4.8) into (4.1) and integrating over $\mathcal{M}_{2}$, one finally obtains the low energy expansion of the amplitude in ten dimensions,

$$
\begin{aligned}
\mathcal{A}_{(4)}^{2-\text { loop }} & =\delta(k)\left(\frac{\alpha^{\prime}}{2}\right)^{3} \frac{\kappa^{4} e^{2 \lambda}}{2^{12} \pi^{6}} K \tilde{K} \int_{\mathcal{M}_{2}} d \mu_{2}\left[\sigma_{2}+2 \varphi \sigma_{3}+\frac{1}{4} A_{1} \sigma_{4}+\mathcal{O}\left(s_{i j}^{5}\right)\right] \\
& =\delta(k)\left(\frac{\alpha^{\prime}}{2}\right)^{3} \frac{\kappa^{4} e^{2 \lambda}}{2^{10} 3^{3} 5 \pi^{3}} K \tilde{K}\left[\sigma_{2}+3 \sigma_{3}+\frac{1}{4} c_{1}(\Lambda) \sigma_{4}+\mathcal{O}\left(s_{i j}^{5}\right)\right]
\end{aligned}
$$

where we used the formula (2.46) for the volume of $\mathcal{M}_{2}$, and the identities

$$
\int_{\mathcal{M}_{2}} d \mu_{2} \varphi=\frac{3}{2} \operatorname{Vol}_{2} \quad \int_{\mathcal{M}_{2}(\Lambda)} d \mu_{2} A_{1}=c_{1}(\Lambda) \operatorname{Vol}_{2}
$$

The coefficient $3 / 2$ in (4.11) was computed in [30] and shown to be consistent with predictions from S-duality. The coefficient $c_{1}(\Lambda)$ depends on an infrared sliding scale $\Lambda$ which is necessary to disentangle the non-local part of the amplitude, which is governed entirely by exchange of massless particles with momentum less than $\Lambda$, from stringy corrections [48, 49].

\subsection{The five-point amplitude}

The genus-two five-point amplitude is given by $[8,12]$

$$
\mathcal{A}_{(5)}^{\text {genus }-2}=\delta(k) \mathcal{N}_{(5)} \int_{\mathcal{M}_{2}} d \mu_{2} \mathcal{B}_{(5)}\left(k_{i} \mid \Omega\right)
$$

with $\mathcal{B}_{(5)}\left(k_{i} \mid \Omega\right)$ defined by (2.49). The overall normalization was obtained using the pure spinor formalism in [8]

$$
\mathcal{N}_{(5)}=\left(\frac{\alpha^{\prime}}{2}\right)^{5} \frac{\kappa^{5} e^{2 \lambda}}{2^{11} \pi^{5}}
$$


As shown in section 3.6, the integrand decomposes as a sum of 4 different types of integrals,

$$
\mathcal{B}_{(5)}=\mathcal{B}_{(5)}^{J}+\mathcal{B}_{(5)}^{F}+\mathcal{B}_{(5)}^{G}+\mathcal{B}_{(5)}^{H}
$$

each one including its own kinematic factor.

\subsubsection{Terms of order $D^{2} \mathcal{R}^{5}$}

At leading order $D^{2} \mathcal{R}^{5}$, the $G$ and $H$ integrals do not contribute, and the remaining integrals are constants, independent of the period matrix $\Omega$,

$$
\begin{array}{lll}
J_{1,1}=128+\mathcal{O}\left(s_{i j}\right), & F_{1}=\frac{128}{s_{12}}+\mathcal{O}\left(s_{i j}^{0}\right), & F_{3}=-\frac{128}{s_{12}}+\mathcal{O}\left(s_{i j}^{0}\right) \\
J_{1,2}=32+\mathcal{O}\left(s_{i j}\right), & F_{2}=\frac{64}{s_{12}}+\mathcal{O}\left(s_{i j}^{0}\right), & F_{4}=-\frac{64}{s_{12}}+\mathcal{O}\left(s_{i j}^{0}\right) \\
J_{1,3}=-64+\mathcal{O}\left(s_{i j}\right), &
\end{array}
$$

Upon using the kinematic identity $T_{1,2,3 \mid 4,5}^{m}=T_{3,4,5 \mid 1,2}^{m}+\operatorname{cycl}(1,2,3)$, the low energy limit of the contraction $\left|\mathcal{V}_{I}^{m}\right|^{2}$ in (3.24) can be rewritten as

$$
\left.\mathcal{B}_{(5)}^{J}\right|_{D^{2} \mathcal{R}^{5}}=64\left(\frac{\alpha^{\prime}}{2}\right) T_{3,4,5 \mid 1,2}^{m} \tilde{T}_{3,4,5 \mid 1,2}^{m}+(1,2 \mid 1,2,3,4,5)
$$

where the notation $+(1,2 \mid 1,2, \ldots, k)$ in the first line instructs to sum over all possibilities to exchange $(1,2)$ by a different pair $(i, j)$ from $i, j \in\{1,2, \ldots, k\}$ for a total of $\left(\begin{array}{l}k \\ 2\end{array}\right)$ terms. Similarly, the integrals in (3.26) to (3.28) produce,

$$
\mathcal{B}_{(5)}^{F}=\frac{64}{s_{12}}\left(\frac{\alpha^{\prime}}{2}\right)^{2}\left[\left|T_{12,3 \mid 4,5}\right|^{2}+\left|T_{12,4 \mid 3,5}\right|^{2}+\left|T_{12,5 \mid 3,4}\right|^{2}\right]+(1,2 \mid 1,2,3,4,5)
$$

and the contributions from the integrals $G$ and $H$ vanish,

$$
\left.\mathcal{B}_{(5)}^{G}\right|_{D^{2} \mathcal{R}^{5}}=\left.\mathcal{B}_{(5)}^{H}\right|_{D^{2} \mathcal{R}^{5}}=0
$$

The expression (4.17) for $\mathcal{B}_{(5)}^{F}$ highlights the benefit of using the OPE-like representation for the second equation in (2.10) since then all terms proportional to $S_{i ; j|k| l, m}$ building blocks trivially cancel as they correspond to non-singular terms on the surface. Hence, at leading order, the integrand (4.14) reduces to,

$$
\begin{aligned}
\left.\mathcal{B}_{(5)}\right|_{D^{2} \mathcal{R}^{5}}= & 64\left(\frac{\alpha^{\prime}}{2}\right)^{2} \frac{\left|T_{12,3 \mid 4,5}\right|^{2}+\left|T_{12,4 \mid 3,5}\right|^{2}+\left|T_{12,5 \mid 3,4}\right|^{2}}{s_{12}} \\
& +64\left(\frac{\alpha^{\prime}}{2}\right) T_{3,4,5 \mid 1,2}^{m} \tilde{T}_{3,4,5 \mid 1,2}^{m}+(1,2 \mid 1,2, \ldots, 5)
\end{aligned}
$$

where the permutations $+(1,2 \mid 1,2, \ldots, 5)$ apply to the entire right side.

This is in agreement with the result obtained in [8], and corresponds to a $D^{2} \mathcal{R}^{5}$ interaction in the low energy effective action, which is expected to be related to $D^{4} \mathcal{R}^{4}$ by non-linear supersymmetry. The residue of the pole in $s_{12}$ is precisely given by the twoparticle superfields $\left|T_{12, i \mid j, k}\right|^{2}$ as is expected from factorization of the 5-point amplitude on a massless external state of two massless states, as shown schematically in figure 2 . 

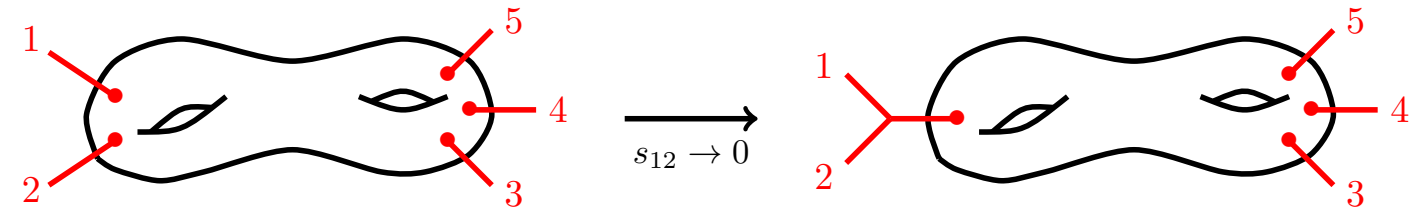

Figure 2. Factorization of the genus-two five-point amplitude onto a massless intermediate state in the $s_{12}$-channel into the tree-level 3-point function and the genus-two 4-point function with massless external states.

\subsubsection{Terms of order $D^{4} \mathcal{R}^{5}$}

At next to leading order $D^{4} \mathcal{R}^{5}$, all integrals are proportional to the Kawazumi-Zhang invariant $\varphi$ in (3.13). The expansions in (3.19) receive contributions from the vector block, and give rise to,

$$
\begin{aligned}
\left.\mathcal{B}_{(5)}^{J}\right|_{D^{4} \mathcal{R}^{5}}= & 128 \varphi\left(s_{35}\left(T_{5,1,2 \mid 3,4}^{m} \tilde{T}_{1,2,3 \mid 4,5}^{m}+T_{1,2,3 \mid 4,5}^{m} \tilde{T}_{5,1,2 \mid 3,4}^{m}\right)\right. \\
& +\left(s_{45}-s_{13}\right)\left(T_{5,1,2 \mid 3,4}^{m} \tilde{T}_{2,3,4 \mid 5,1}^{m}+T_{2,3,4 \mid 5,1}^{m} \tilde{T}_{5,1,2 \mid 3,4}^{m}\right) \\
& \left.-\left(s_{23}+s_{45}\right) T_{5,1,2 \mid 3,4}^{m} \tilde{T}_{5,1,2 \mid 3,4}^{m}+\operatorname{cycl}(1,2,3,4,5)\right) \\
= & 128 \varphi s_{12} T_{3,4,5 \mid 1,2}^{m} \tilde{T}_{3,4,5 \mid 1,2}^{m}+(1,2 \mid 1,2,3,4,5)
\end{aligned}
$$

The expansions in (3.20) produce all the singular terms, and give rise to,

$$
\begin{aligned}
\left.\mathcal{B}_{12}^{F}\right|_{D^{4} \mathcal{R}^{5}}= & \frac{128 \varphi}{s_{12}}\left(s_{34}\left|T_{12,5 \mid 3,4}\right|^{2}+s_{35}\left|T_{12,4 \mid 3,5}\right|^{2}+s_{45}\left|T_{12,3 \mid 4,5}\right|^{2}\right) \\
& -32 \varphi\left(\left|S_{1 ; 2|3| 4,5}\right|^{2}+\left|S_{1 ; 2|4| 3,5}\right|^{2}+\left|S_{1 ; 2|5| 3,4}\right|^{2}\right. \\
& \left.+\left|S_{2 ; 1|3| 4,5}\right|^{2}+\left|S_{2 ; 1|4| 3,5}\right|^{2}+\left|S_{2 ; 1|5| 3,4}\right|^{2}\right)
\end{aligned}
$$

Finally, the contributions in (3.21) and (3.22) are analytic in $s_{i j}$ and receive contributions from the scalar block, and give rise to,

$$
\begin{aligned}
\left.\mathcal{B}_{1,23}^{G}\right|_{D^{4} \mathcal{R}^{5}} & =-32 \varphi\left(S_{1 ; 2|4| 3,5} \tilde{S}_{1 ; 3|4| 2,5}+S_{1 ; 2|5| 3,4} \tilde{S}_{1 ; 3|5| 2,4}\right) \\
\left.\mathcal{B}_{12,34}^{H}\right|_{D^{4} \mathcal{R}^{5}} & =0
\end{aligned}
$$

Whenever possible, the expressions have been simplified by repeatedly applying the relations $T_{12,3 \mid 4,5}=S_{1 ; 2|3| 4,5}-S_{2 ; 1|3| 4,5}$ and $S_{1 ; 2|3| 4,5}+\operatorname{cycl}(3,4,5) \cong 0$.

Adding up these contributions according to (3.25), we get,

$$
\begin{aligned}
\left.\mathcal{B}_{(5)}\right|_{D^{4} \mathcal{R}^{5}}= & 128 \varphi\left[\left(\frac{\alpha^{\prime}}{2}\right) s_{45} T_{1,2,3 \mid 4,5}^{m} \tilde{T}_{1,2,3 \mid 4,5}^{m}+(4,5 \mid 1,2,3,4,5)\right. \\
& +\left(\frac{\alpha^{\prime}}{2}\right)^{2}\left(\frac{s_{12}}{s_{45}}\left|T_{45,3 \mid 1,2}\right|^{2}+\frac{s_{13}}{s_{45}}\left|T_{45,2 \mid 1,3}\right|^{2}+\frac{s_{23}}{s_{45}}\left|T_{45,1 \mid 2,3}\right|^{2}+(4,5 \mid 1,2,3,4,5)\right) \\
& -\left(\frac{\alpha^{\prime}}{2}\right)^{2}\left(\left|S_{1 ; 2|3| 4,5}+S_{1 ; 3|2| 4,5}\right|^{2}+\left|S_{1 ; 2|4| 3,5}+S_{1 ; 4|2| 3,5}\right|^{2}\right. \\
& \left.\left.+\left|S_{1 ; 3|4| 2,5}+S_{1 ; 4|3| 2,5}\right|^{2}+(1 \leftrightarrow 2,3,4,5)\right)\right]
\end{aligned}
$$


where the last two relations of (2.23) have been used to simplify the sums over permutations of (4.21) and (4.22).

\subsubsection{Terms of order $D^{6} \mathcal{R}^{5}$}

In contrast to the lower-order terms in the previous subsections, the low energy expansion of the genus-two amplitude at the order $D^{6} \mathcal{R}^{5}$ features linearly independent MGFs $\mathcal{Z}_{1}, \mathcal{Z}_{2}, \mathcal{Z}_{3}, \mathcal{Z}_{5}$ and $\varphi^{2}$ defined in (3.14). Their respective coefficients are given by BRSTinvariant linear combinations of the building blocks that compose the correlator. As usual in such situations, the resulting expressions that arise after expanding the integrals are not necessarily in the most compact form. However, utilizing various cohomology manipulations in pure spinor superspace as done in (4.23), it may be possible to simplify the answer after trial and error.

First, it is beneficial to rewrite the five MGFs in terms of the linear combinations $A_{1}$ and $A_{2}$ identified in the four-point calculations in section 4.1 , along with $\mathcal{Z}_{1}, \mathcal{Z}_{5}$ and $\varphi^{2}$. In doing so the number of terms reduce by approximately $10 \%$. We shall now display the coefficients of $A_{1}$ and $\mathcal{Z}_{5}$.

Curiously, the BRST-invariant coefficient of $A_{1}$ at $D^{6} \mathcal{R}^{5}$ turns out to be closely related to the coefficient of $\varphi$ at order $D^{4} \mathcal{R}^{5}$ given in (4.23). In fact, one can show that

$$
\begin{aligned}
\left.\mathcal{B}_{(5)}\right|_{A_{1}}= & 16\left[\left(\frac{\alpha^{\prime}}{2}\right) s_{45}^{2} T_{1,2,3 \mid 4,5}^{m} \tilde{T}_{1,2,3 \mid 4,5}^{m}+(4,5 \mid 1,2,3,4,5)\right. \\
& +\left(\frac{\alpha^{\prime}}{2}\right)^{2}\left(\frac{s_{12}^{2}}{s_{45}}\left|T_{45,3 \mid 1,2}\right|^{2}+\frac{s_{13}^{2}}{s_{45}}\left|T_{45,2 \mid 1,3}\right|^{2}+\frac{s_{23}^{2}}{s_{45}}\left|T_{45,1 \mid 2,3}\right|^{2}+(4,5 \mid 1,2,3,4,5)\right) \\
& -\left(\frac{\alpha^{\prime}}{2}\right)^{2}\left(\left(s_{23}+s_{45}\right)\left|S_{1 ; 2|3| 4,5}+S_{1 ; 3|2| 4,5}\right|^{2}+\left(s_{24}+s_{35}\right)\left|S_{1 ; 2|4| 3,5}+S_{1 ; 4|2| 3,5}\right|^{2}\right. \\
& \left.\left.+\left(s_{34}+s_{25}\right)\left|S_{1 ; 3|4| 2,5}+S_{1 ; 4|3| 2,5}\right|^{2}+(1 \leftrightarrow 2,3,4,5)\right)\right]
\end{aligned}
$$

Given that $\mathcal{Z}_{5}$ defined by the fifth line of (3.14) only appears in the $G_{2}$ integral (3.21), its overall coefficient is easily assembled from (3.27) and (3.25):

$$
\begin{aligned}
\left.\mathcal{B}_{(5)}\right|_{\mathcal{Z}_{5}}= & \left.\sum_{2 \leq i<j}^{5}\left(\mathcal{B}_{1 ; i j}^{G}+\mathcal{B}_{1 ; j i}^{G}\right)\right|_{\mathcal{Z}_{5}}+(1 \leftrightarrow 2,3,4,5) \\
= & 4 s_{12}\left(S_{1 ; 3|5| 2,4} \tilde{S}_{1 ; 4|5| 2,3}+S_{1 ; 4|5| 2,3} \tilde{S}_{1 ; 3|5| 2,4}-S_{1 ; 3|2| 4,5} \tilde{S}_{1 ; 4|2| 3,5}-S_{1 ; 4|2| 3,5} \tilde{S}_{1 ; 3|2| 4,5}\right) \\
& +4 s_{13}\left(S_{1 ; 2|5| 3,4} \tilde{S}_{1 ; 4|5| 2,3}+S_{1 ; 4|5| 2,3} \tilde{S}_{1 ; 2|5| 3,4}-S_{1 ; 2|3| 4,5} \tilde{S}_{1 ; 4|3| 2,5}-S_{1 ; 4|3| 2,5} \tilde{S}_{1 ; 2|3| 4,5}\right) \\
& +4 s_{14}\left(S_{1 ; 2|5| 3,4} \tilde{S}_{1 ; 3|5| 2,4}+S_{1 ; 3|5| 2,4} \tilde{S}_{1 ; 2|5| 3,4}-S_{1 ; 2|4| 3,5} \tilde{S}_{1 ; 3|4| 2,5}-S_{1 ; 3|4| 2,5} \tilde{S}_{1 ; 2|4| 3,5}\right) \\
& +(1 \leftrightarrow 2,3,4,5)
\end{aligned}
$$

Alternatively, one can recast (4.25) in terms of Yang-Mills tree amplitudes as detailed in the next subsection. To do so one uses the conversion to genus-two BRST invariants $S_{a ; b|c| d, e} \rightarrow s_{a b} C_{a ; b|c| d, e}$, and the cohomology identity (2.28) to rewrite $C_{a ; b|c| d, e}$ in terms of genus-one BRST invariants, and finally use (2.29) to convert to Yang-Mills tree amplitudes. 
In doing this one obtains that the coefficient of $\mathcal{Z}_{5}$ is proportional to $\mathcal{B}_{\left\{7^{\prime}\right\}}^{\text {!tree }}$ to be defined in $(4.54)$,

$$
\left.\mathcal{B}_{(5)}\right|_{\mathcal{Z}_{5}}=-2560 \mathcal{B}_{\left\{7^{\prime}\right\}}^{\text {!tree }}
$$

This result is interesting because the representation (4.25) is manifestly local while the locality of $\mathcal{B}_{\left\{7^{\prime}\right\}}^{\text {!tree }}$ is not evident. In addition, the above steps can be used to derive the expression of the $2 \times 2$ matrix $M_{7}^{\prime}$ in (4.54) algorithmically, and it would be rewarding to look for similar derivations of other $M_{n}^{\prime}$ matrices.

The coefficients of $A_{2}, \mathcal{Z}_{1}$ and $\varphi^{2}$ can be brought into a form similar to (4.24) and (4.25) and can be downloaded from [63]. Their components for external Type IIA and IIB states will be further simplified in the next subsections.

\subsection{Components in Type IIB}

Here we shall express the components of the genus-two amplitude of the ten-dimensional Type IIB superstring in terms of color-ordered tree-level amplitudes $A_{\mathrm{YM}}(1,2,3,4,5)$ of ten-dimensional SYM. For this it is convenient to use the representation (2.13) of the genustwo correlator written in terms of the BRST invariants $C_{1,2,3 \mid 4,5}^{m}$ and $C_{1 ; 2|3| 4,5}$. As reviewed in section 2.3.2, these genus-two invariants can be expressed in terms of the genus-one five-point BRST invariants $C_{1 \mid 2,3,4,5}^{m}$ and $C_{1 \mid 23,4,5}$. The scalar genus-one invariants $C_{1 \mid 23,4,5}$ occurring in the $|\mathcal{W}|^{2}$ part of the genus-two correlator were shown in [61] to be equivalent to SYM tree amplitudes, see (2.29). This relation holds for the entire massless multiplets of both Type IIB and IIA.

To relate the vector invariants $C_{1 \mid 2,3,4,5}^{m}$ occurring in the $\left|\mathcal{V}_{I}^{m}\right|^{2}$ part of the amplitude to SYM tree-level amplitudes, we use an observation from [35], which holds only for Type IIB: even though an individual genus-one invariant $C_{1 \mid 2,3,4,5}^{m}$ cannot be written in terms of $A_{\mathrm{YM}}$, the left-right holomorphic square $\left\langle\left|C_{1 \mid 2,3,4,5}^{m}\right|^{2}\right\rangle_{0}$ can in fact be written in terms of $A_{\mathrm{YM}} \tilde{A}_{\mathrm{YM}}$ provided the external states are five gravitons or four gravitons and one dilaton of Type IIB. More explicitly [35] (up to an overall normalization), we have,

$$
\begin{aligned}
& \left.\left\langle C_{1 \mid 2,3,4,5}^{m} \tilde{C}_{1 \mid 2,3,4,5}^{m}+\left[s_{23} C_{1 \mid 23,4,5} \tilde{C}_{1 \mid 23,4,5}+(2,3 \mid 2,3,4,5)\right]\right\rangle_{0}\right|_{\mathrm{IIB}} \\
& =\tilde{A}_{54}^{T} \cdot S_{0} \cdot M_{3} \cdot A_{45} \times\left\{\begin{aligned}
1: & \text { five gravitons } \\
-\frac{1}{3}: & \text { four gravitons and one dilaton }
\end{aligned}\right.
\end{aligned}
$$

where the two options depend on the total $\mathrm{U}(1)_{R}$ charge of the external states, 0 or \pm 2 , respectively. ${ }^{17}$ By linearized supersymmetry, the kinematic relation (4.27) extends to the remaining massless Type IIB state configurations with the same $\mathrm{U}(1)_{R}$ charges. For instance, the first line also applies to three gravitons and two gravitini of opposite $\mathrm{U}(1)_{R}$ charges, and the second one to three gravitons, one gravitino and dilatino whose $\mathrm{U}(1)_{R}$ charges have the same sign.

\footnotetext{
${ }^{17}$ Due to symmetry under worldsheet parity, which acts by $(-1)^{Q}$ on a field with $\mathrm{U}(1)_{R}$ charge $Q$, the $\mathrm{U}(1)_{R}$ symmetry can only be violated by an even number. In particular, the four-graviton, one KalbRamond amplitude would violate $\mathrm{U}(1)_{R}$ by \pm 1 units and therefore must vanish at all genera, as explained in [75]. We have verified that this is indeed the case at genus-two up to order $D^{6} \mathcal{R}^{5}$.
} 
Since the scalar invariants $\left\langle\left|C_{1 \mid 23,4,5}\right|^{2}\right\rangle_{0}$ can be expanded in terms of $A_{\mathrm{YM}} \tilde{A}_{\mathrm{YM}}$ it follows that for the genus-two five-graviton and four-graviton-one-dilaton amplitudes in Type IIB, $\left\langle\left|C_{1 \mid 2,3,4,5}^{m}\right|^{2}\right\rangle_{0}$, can also be written in terms of SYM tree amplitudes. Such a relation does not exist for five-point amplitude of gravitons and dilatons in Type IIA.

In (4.27) we have used the following notation for the two-component vectors of SYM amplitudes that form bases of $\tilde{A}_{\mathrm{YM}}$ and $A_{\mathrm{YM}}$ under BCJ relations [76]

$$
\tilde{A}_{54}=\left(\begin{array}{c}
\tilde{A}_{\mathrm{YM}}(1,2,3,5,4) \\
\tilde{A}_{\mathrm{YM}}(1,3,2,5,4)
\end{array}\right) \quad A_{45}=\left(\begin{array}{c}
A_{\mathrm{YM}}(1,2,3,4,5) \\
A_{\mathrm{YM}}(1,3,2,4,5)
\end{array}\right)
$$

Furthermore, (4.27) features the field-theory momentum kernel of [77],

$$
S_{0}=\left(\begin{array}{cc}
\left(k_{1} \cdot k_{2}\right)\left(k_{12} \cdot k_{3}\right) & \left(k_{1} \cdot k_{2}\right)\left(k_{1} \cdot k_{3}\right) \\
\left(k_{1} \cdot k_{2}\right)\left(k_{1} \cdot k_{3}\right) & \left(k_{1} \cdot k_{3}\right)\left(k_{13} \cdot k_{2}\right)
\end{array}\right)
$$

with $k_{i j}=k_{i}+k_{j}$, while the matrix $M_{3}$ encoding the $\alpha^{\prime 3}$ corrections to open- and closedsuperstring tree-level amplitudes is given by [34],

$$
M_{3}=\left(\begin{array}{cc}
m_{11} & m_{12} \\
\left.m_{12}\right|_{2 \leftrightarrow 3} & \left.m_{11}\right|_{2 \leftrightarrow 3}
\end{array}\right)
$$

where the permutation inequivalent components are given as follows,

$$
\begin{aligned}
& m_{11}=s_{34}\left[s_{45}^{2}+s_{34} s_{45}-s_{12}\left(s_{12}+2 s_{23}+s_{34}\right)\right]+s_{12} s_{15}\left(s_{12}+s_{15}\right) \\
& m_{12}=-s_{13} s_{24}\left(s_{12}+s_{23}+s_{34}+s_{45}+s_{15}\right)
\end{aligned}
$$

Based on (2.29) and (4.27), the entire polarization dependence of massless five-point genustwo amplitudes in Type IIB superstrings can be reduced to products $A_{\mathrm{YM}} \tilde{A}_{\mathrm{YM}}$ as for tree level $[34,78]$ and genus one [35]. The relative factor of $-\frac{1}{3}$ in $(4.27)$ between the $\mathrm{U}(1)_{R^{-}}$

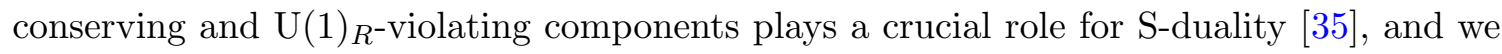
will elaborate on its genus-two analogue in section 5 .

\subsubsection{Five-point tree-level amplitudes of SYM}

We shall now review a compact way of representing the polarization dependence of the color-ordered five-point SYM amplitudes in (4.28). Following the recursive strategy of Berends and Giele [79], five-point SYM amplitudes can be efficiently organized in terms of two-particle polarizations $\varepsilon_{12}^{m}, f_{12}^{m n}$ and $\chi_{12}^{\alpha}$,

$$
\begin{aligned}
\varepsilon_{12}^{m} & =i \varepsilon_{2}^{m}\left(k_{2} \cdot \varepsilon_{1}\right)-i \varepsilon_{1}^{m}\left(k_{1} \cdot \varepsilon_{2}\right)+\frac{i}{2}\left(k_{1}^{m}-k_{2}^{m}\right)\left(\varepsilon_{1} \cdot \varepsilon_{2}\right)+\left(\chi_{1} \gamma^{m} \chi_{2}\right) \\
f_{12}^{m n} & =\varepsilon_{12}^{m} k_{12}^{n}-\varepsilon_{12}^{n} k_{12}^{m}+i k_{1} \cdot k_{2}\left(\varepsilon_{1}^{m} \varepsilon_{2}^{n}-\varepsilon_{1}^{n} \varepsilon_{2}^{m}\right) \\
\chi_{12}^{\alpha} & =\frac{i}{2} k_{12}^{m} \gamma_{m}^{\alpha \beta}\left[\varepsilon_{1}^{p}\left(\gamma_{p} \chi_{2}\right)_{\beta}-\varepsilon_{2}^{p}\left(\gamma_{p} \chi_{1}\right)_{\beta}\right]
\end{aligned}
$$


In terms of these data, the five-point SYM amplitude obtained from the superspace expression [80] is given by [81],

$$
\begin{aligned}
A_{\mathrm{YM}}(1,2,3,4,5)= & \frac{i}{2 s_{12} s_{34}}\left[\varepsilon_{12}^{m} f_{34}^{m n} \varepsilon_{5}^{n}+\varepsilon_{34}^{m} f_{5}^{m n} \varepsilon_{12}^{n}+\varepsilon_{5}^{m} f_{12}^{m n} \varepsilon_{34}^{n}\right] \\
& +\frac{1}{s_{12} s_{34}}\left[\left(\chi_{12} \gamma_{m} \chi_{34}\right) \varepsilon_{5}^{m}+\left(\chi_{34} \gamma_{m} \chi_{5}\right) \varepsilon_{12}^{m}+\left(\chi_{5} \gamma_{m} \chi_{12}\right) \varepsilon_{34}^{m}\right] \\
& +\operatorname{cycl}(1,2,3,4,5)
\end{aligned}
$$

Note that both lines of (4.33) contribute to gluino amplitudes since $\varepsilon_{i j}^{m}$ and $f_{i j}^{m n}$ contain a term bilinear in $\chi_{i}^{\alpha}, \chi_{j}^{\beta}$.

\subsection{Components in Type IIA}

We now turn to the case of Type IIA superstrings, where the Weyl spinors of the left and right movers have opposite chirality. While the previous relation (4.27) between vector blocks and SYM tree amplitudes no longer works, the difference between the coefficients of these blocks in Type IIA and Type IIB has a very simple structure, which amounts to flipping the sign of the ten-dimensional Levi-Civita symbol $\epsilon_{10}$ appearing in $\tilde{T}_{1,2,3 \mid 4,5}^{m}[63]$ :

$$
\begin{aligned}
\left\langle\tilde{T}_{1,2,3 \mid 4,5}^{m}\right\rangle_{0}= & \frac{1}{2880} s_{45}\left[\tilde{\varepsilon}_{1}^{m} t_{8}\left(\tilde{f}_{2}, \tilde{f}_{3}, \tilde{f}_{4}, \tilde{f}_{5}\right)+(1 \leftrightarrow 2,3,4,5)\right]+\sum_{j=2}^{5} k_{j}^{m} \tilde{T}_{j} \\
& -\frac{1}{5760} s_{45} \epsilon_{10}^{m}\left(\tilde{\varepsilon}_{1}, \tilde{f}_{2}, \tilde{f}_{3}, \tilde{f}_{4}, \tilde{f}_{5}\right) \times\left\{\begin{array}{l}
+1 \text { (Type IIB) } \\
-1 \text { (Type IIA) }
\end{array}\right.
\end{aligned}
$$

Here, we have used the shorthand $t_{8}\left(f_{2}, f_{3}, f_{4}, f_{5}\right)$ as in (2.31) as well as,

$$
\epsilon_{10}^{m}\left(\varepsilon_{1}, f_{2}, f_{3}, f_{4}, f_{5}\right)=\left(\epsilon_{10}\right)^{m}{ }_{n p_{2} q_{2} p_{3} q_{3} p_{4} q_{4} p_{5} q_{5}} \varepsilon_{1}^{n} f_{2}^{p_{2} q_{2}} f_{3}^{p_{3} q_{3}} f_{4}^{p_{4} q_{4}} f_{5}^{p_{5} q_{5}}
$$

where $f_{i}$ denotes the linearized field-strength $f_{i}^{m n}=\varepsilon_{i}^{m} k_{i}^{n}-k_{i}^{m} \varepsilon_{i}^{n}$ of external state $i$. The form of the scalar terms $\tilde{T}_{j}$ in the first line of (4.34) will not be relevant in the discussions below due to the vanishing contraction $k_{j}^{m} \epsilon_{10}^{m}\left(\tilde{\varepsilon}_{1}, \tilde{f}_{2}, \tilde{f}_{3}, \tilde{f}_{4}, \tilde{f}_{5}\right)=0$ for $j=1,2,3,4,5$.

Therefore, the difference between Type IIA and Type IIB correlators may be inferred from the following simple relation,

$$
\left.\left\langle\tilde{T}_{1,2,3 \mid 4,5}^{m}\right\rangle_{0}\right|_{\mathrm{IIA}}=\left.\left\langle\tilde{T}_{1,2,3 \mid 4,5}^{m}\right\rangle_{0}\right|_{\mathrm{IIB}}+\frac{s_{45}}{2880} \epsilon_{10}^{m}\left(\tilde{\varepsilon}_{1}, \tilde{f}_{2}, \tilde{f}_{3}, \tilde{f}_{4}, \tilde{f}_{5}\right)
$$

and we obtain,

$$
\begin{aligned}
& \left.\left\langle\mathcal{W} \tilde{\mathcal{W}}-\left(\frac{\alpha^{\prime}}{2}\right) \pi Y^{I J} \mathcal{V}_{I}^{m} \tilde{\mathcal{V}}_{J}^{m}\right\rangle_{0}\right|_{\text {IIB }}-\left.\left\langle\mathcal{W} \tilde{\mathcal{W}}-\left(\frac{\alpha^{\prime}}{2}\right) \pi Y^{I J} \mathcal{V}_{I}^{m} \tilde{\mathcal{V}}_{J}^{m}\right\rangle_{0}\right|_{\text {IIA }} \\
& =\frac{1}{2880}\left(\frac{\alpha^{\prime}}{2}\right) \pi Y^{I J}\left\langle\mathcal{V}_{I}^{m}\right\rangle_{0} \epsilon_{10}^{m}\left(\tilde{\varepsilon}_{1}, \tilde{f}_{2}, \tilde{f}_{3}, \tilde{f}_{4}, \tilde{f}_{5}\right)\left[s_{45} \bar{\omega}_{J}(2) \overline{\Delta(3,4) \Delta(5,1)}+\operatorname{cycl}(1,2,3,4,5)\right]
\end{aligned}
$$

see (2.10) for the cyclic permutations of $T_{1,2,3 \mid 4,5}^{m}$ entering $\mathcal{V}_{I}^{m}$. As will be detailed below, the contributions from $\left\langle\mathcal{V}_{I}^{m}\right\rangle_{0} \epsilon_{10}^{m}\left(\tilde{\varepsilon}_{1}, \tilde{f}_{2}, \tilde{f}_{3}, \tilde{f}_{4}, \tilde{f}_{5}\right)$ take different forms depending on the type of 
external NSNS states. We will show that, both for five gravitons and for four gravitons and one Kalb-Ramond $B$-field, the difference between Type IIB and Type IIA amplitudes is proportional to the integral,

$$
\begin{aligned}
\mathcal{J}_{\text {IIA }}= & \frac{i}{2} \int_{\Sigma^{5}} \frac{\mathrm{KN}_{(5)}}{(\operatorname{det} Y)^{2}} Y^{I J}\left[s_{45} \omega_{I}(2) \Delta(3,4) \Delta(5,1)+\operatorname{cycl}(1,2,3,4,5)\right] \\
& \times\left[s_{45} \bar{\omega}_{J}(2) \overline{\Delta(3,4) \Delta(5,1)}+\operatorname{cycl}(1,2,3,4,5)\right]
\end{aligned}
$$

This integral is invariant under permutations of all external legs, and can be expressed in terms of the $J$-integrals defined in (3.3)

$$
\mathcal{J}_{\text {IIA }}=J_{1,1} s_{34}^{2}+\left(J_{1,2}+\overline{J_{1,2}}\right) s_{34} s_{45}+\left(J_{1,3}+\overline{J_{1,3}}\right) s_{34} s_{15}+\operatorname{cycl}(1,2,3,4,5)
$$

The low energy expansion of this integral follows immediately from (3.19),

$$
\begin{aligned}
\mathcal{J}_{\text {IIA }}= & 32 \sum_{1 \leq i<j}^{5} s_{i j}^{2}+64 \varphi \sum_{1 \leq i<j}^{5} s_{i j}^{3}+\frac{8}{3}\left(-\mathcal{Z}_{1}-8 \mathcal{Z}_{2}-\mathcal{Z}_{3}-\mathcal{Z}_{4}+10 \varphi^{2}\right) \sum_{1 \leq i<j}^{5} s_{i j}^{4} \\
& +\frac{2}{3}\left(4 \mathcal{Z}_{1}+2 \mathcal{Z}_{2}+4 \mathcal{Z}_{3}+\mathcal{Z}_{4}-10 \varphi^{2}\right)\left(\sum_{1 \leq i<j}^{5} s_{i j}^{2}\right)^{2}+\mathcal{O}\left(s_{i j}^{5}\right) \\
= & 32 P_{2}+64 \varphi P_{3}+\frac{8}{3}\left(\mathcal{Z}_{1}-6 \mathcal{Z}_{2}+\mathcal{Z}_{3}+8 \varphi^{2}\right) P_{4}+\frac{4}{3}\left(\mathcal{Z}_{1}+\mathcal{Z}_{3}-4 \varphi^{2}\right) P_{2}^{2}+\mathcal{O}\left(s_{i j}^{5}\right)
\end{aligned}
$$

where $P_{n}$ denotes the symmetric homogeneous polynomials ${ }^{18}$

$$
P_{n}=\sum_{1 \leq i<j}^{5} s_{i j}^{n}
$$

\subsubsection{Five gravitons in Type IIA}

For five external gravitons (or more generally for external states with $\varepsilon_{i}=\tilde{\varepsilon}_{i}$ ), the kinematic factors $\left\langle\mathcal{V}_{I}^{m}\right\rangle_{0} \epsilon_{10}^{m}\left(\tilde{\varepsilon}_{1}, \tilde{f}_{2}, \tilde{f}_{3}, \tilde{f}_{4}, \tilde{f}_{5}\right)$ in (4.37) only receive contributions from,

$$
\left\langle T_{1,2,3 \mid 4,5}^{m}\right\rangle_{0} \epsilon_{10}^{m}\left(\tilde{\varepsilon}_{1}, \tilde{f}_{2}, \tilde{f}_{3}, \tilde{f}_{4}, \tilde{f}_{5}\right) \rightarrow-\frac{1}{5760} s_{45} \epsilon_{10}^{m}\left(\varepsilon_{1}, f_{2}, f_{3}, f_{4}, f_{5}\right) \epsilon_{10}^{m}\left(\varepsilon_{1}, f_{2}, f_{3}, f_{4}, f_{5}\right)
$$

since terms of the form $\epsilon_{10}\left(\varepsilon_{j}, \tilde{\varepsilon}_{1}, \tilde{f}_{2}, \tilde{f}_{3}, \tilde{f}_{4}, \tilde{f}_{5}\right)$ from the first line of (4.34) vanish due to the symmetry of the graviton polarization tensors under $\tilde{\varepsilon}_{j} \leftrightarrow \varepsilon_{j}$. Hence, the difference between Type IIB and Type IIA integrands reduces to,

$$
\left.\mathcal{B}_{(5)}\right|_{\mathrm{IIB}} ^{h^{5}}-\left.\mathcal{B}_{(5)}\right|_{\mathrm{IIA}} ^{h^{5}}=\left(\frac{\alpha^{\prime}}{2}\right) \frac{2}{5760^{2}} \epsilon_{10}^{m}\left(\varepsilon_{1}, f_{2}, f_{3}, f_{4}, f_{5}\right) \epsilon_{10}^{m}\left(\varepsilon_{1}, f_{2}, f_{3}, f_{4}, f_{5}\right) \mathcal{J}_{\mathrm{IIA}}
$$

Since $\mathcal{J}_{\text {IIA }}$ behaves as $32 \sum_{1 \leq i<j}^{5} s_{i j}^{2}$ in the low energy limit, the expression (4.43) reproduces, up to an overall constant, the result (5.47) of [8]. The complete Type IIA fivegraviton amplitude can be assembled from (4.43) and from the Type IIB components that are expressible in terms of SYM tree amplitudes by the discussion in section 4.3. The same conclusion holds for any five-point amplitude involving gravitons and dilatons, since it only depends on the symmetry property under $\varepsilon_{j} \leftrightarrow \tilde{\varepsilon}_{j}$.

\footnotetext{
${ }^{18}$ As pointed out in [82], the ring of symmetric polynomials in the $s_{i j}$ 's subject to the momentum conservation constraint is generated by the polynomials $P_{2}, P_{3}, \ldots, P_{9}$ along with an additional degree 6 generator, which we shall not encounter at the order that we work in this paper.
} 


\subsubsection{Four gravitons and one $B$-field in Type IIA}

For Type IIA amplitudes with four external gravitons and one $B$-field in the first leg with polarization $B_{1}^{m n}=\frac{1}{2}\left(\varepsilon_{1}^{m} \tilde{\varepsilon}_{1}^{n}-\varepsilon_{1}^{n} \tilde{\varepsilon}_{1}^{m}\right)$, the kinematic factors $\left\langle\mathcal{V}_{I}^{m}\right\rangle_{0} \epsilon_{10}^{m}\left(\tilde{\varepsilon}_{1}, \tilde{f}_{2}, \tilde{f}_{3}, \tilde{f}_{4}, \tilde{f}_{5}\right)$ in (4.37) only receive contributions from

$$
\left\langle T_{1,2,3 \mid 4,5}^{m}\right\rangle_{0} \epsilon_{10}^{m}\left(\tilde{\varepsilon}_{1}, \tilde{f}_{2}, \tilde{f}_{3}, \tilde{f}_{4}, \tilde{f}_{5}\right) \rightarrow \frac{1}{2880} s_{45} \epsilon_{10}\left(B_{1}, f_{2}, f_{3}, f_{4}, f_{5}\right) t_{8}\left(f_{2}, f_{3}, f_{4}, f_{5}\right)
$$

with shorthand

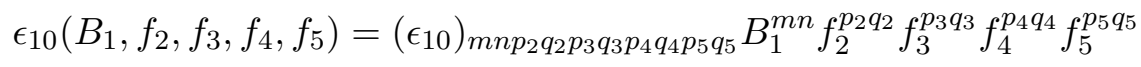

and the $t_{8}$-tensor in (2.31).

Since the Type IIB amplitude involving four gravitons and one $B$-field vanishes to all orders in $\alpha^{\prime}$ (see footnote 17), we conclude from (4.37) that the integrand in Type IIA is,

$$
\left.\mathcal{B}_{(5)}\right|_{\mathrm{IIA}} ^{B h^{4}}=-\left(\frac{\alpha^{\prime}}{2}\right) \frac{1}{2880^{2}} \epsilon_{10}\left(B_{1}, f_{2}, f_{3}, f_{4}, f_{5}\right) t_{8}\left(f_{2}, f_{3}, f_{4}, f_{5}\right) \mathcal{J}_{\text {IIA }}
$$

\subsection{Type IIB 5-point amplitudes up to genus-two}

In this section we verify that the $\alpha^{\prime}$ expansion of the Type IIB five-point amplitude at genus two leads to the same kinematic factors appearing in the expansion of the same amplitude at tree level and genus one.

The properly normalized five-point amplitudes at tree level, genus one and genus two are given by $[8]^{19}$

$$
\begin{aligned}
\mathcal{A}_{(5)}^{\text {tree }} & =\delta(k)\left(\frac{\alpha^{\prime}}{2}\right) \kappa^{5} e^{-2 \lambda}(2 \pi)^{2} \mathcal{B}_{(5)}^{\text {tree }}\left(k_{i}\right), \\
\mathcal{A}_{(5)}^{\text {genus-1 }} & =\delta(k)\left(\frac{\alpha^{\prime}}{2}\right) \frac{\kappa^{5}}{2^{5} \pi} \int_{\mathcal{M}_{1}} d \mu_{1} \mathcal{B}_{(5)}^{\text {genus }-1}\left(k_{i} \mid \tau\right), \\
\mathcal{A}_{(5)}^{\text {genus }-2} & =\delta(k)\left(\frac{\alpha^{\prime}}{2}\right) \frac{\kappa^{5} e^{2 \lambda}}{2^{11} \pi^{5}} \int_{\mathcal{M}_{2}} d \mu_{2} \mathcal{B}_{(5)}^{\text {genus }-2}\left(k_{i} \mid \Omega\right)
\end{aligned}
$$

where $\left[\mathcal{B}_{(5)}^{\text {tree }}\right]=\left[\mathcal{B}_{(5)}^{\text {genus }-1}\right]=\left[\mathcal{B}_{(5)}^{\text {genus }-2}\right]=-2$,

$$
\begin{aligned}
\mathcal{B}_{(5)}^{\text {tree }}\left(k_{i}\right) & =\mathcal{B}_{\{0\}}^{\text {tree }}+\mathcal{B}_{\{3\}}^{\text {tree }} \zeta_{3}+\mathcal{B}_{\{5\}}^{\text {tree }} \zeta_{5}+\mathcal{B}_{\{3,3\}}^{\text {tree }} \zeta_{3}^{2}+\mathcal{B}_{\{7\}}^{\text {tree }} \zeta_{7}+\cdots \\
\mathcal{B}_{(5)}^{\text {genus }-1}\left(k_{i} \mid \tau\right) & =\mathcal{B}_{\{3\}}^{\text {genus }-1}+\mathcal{B}_{\{5\}}^{\text {genus }-1}+\mathcal{B}_{\{3,3\}}^{\text {genus }-1}+\mathcal{B}_{\{7\}}^{\text {genus }-1}+\mathcal{B}_{\left\{7^{\prime}\right\}}^{\text {genus }-1}+\cdots \\
\mathcal{B}_{(5)}^{\text {genus }-2}\left(k_{i} \mid \Omega\right) & =\mathcal{B}_{\{5\}}^{\text {genus }-2}+\mathcal{B}_{\{3,3\}}^{\text {genus }-2}+\mathcal{B}_{\{7\}}^{\text {genus }-2}+\mathcal{B}_{\left\{7^{\prime}\right\}}^{\text {genus }-2}+\cdots
\end{aligned}
$$

and the terms in the ellipsis take the schematic form $s_{i j}^{m} A_{\mathrm{YM}} \tilde{A}_{\mathrm{YM}}$ with $m \geq 8$. As will become clear below, the notation for the subscripts of $\mathcal{B}_{\{\ldots\}}^{\text {tree }}, \mathcal{B}_{\{\ldots\}}^{\text {genus }-1}$ and $\mathcal{B}_{\{\ldots\}}^{\text {genus }-2}$ indicates the polynomial dependence on $s_{i j}$ occuring at different genera.

\footnotetext{
${ }^{19}$ In the conventions of [8] the $n$-point amplitudes have no length dimension independently of loop order; $\left[\mathcal{A}_{(n)}\right]=0$. Note that $\left[\alpha^{\prime}\right]=2,[\kappa]=-2,\left[\delta^{10}(k)\right]=10,\left[k^{m}\right]=-1$ and $\left[\varepsilon_{i}^{m}\right]=0$. In addition we absorbed a common factor of $2^{9} 5^{2}\left(2 / \alpha^{\prime}\right)^{2}$ in the expressions for the various interactions in $\mathcal{B}^{\text {genus-1 }}$ into the overall coefficient of the genus-one amplitude from [8], namely $\left(\alpha^{\prime} / 2\right)^{3} \frac{\kappa^{5}}{2^{14} 5^{2} \pi}$. Similarly a factor of $2^{34} 3^{6} 5^{2}\left(2 / \alpha^{\prime}\right)^{4}$ from $\mathcal{B}^{\text {genus-2 }}$ was absorbed into the overall coefficient $\left(\alpha^{\prime} / 2\right)^{5} \frac{\kappa^{5} e^{2 \lambda}}{2^{45} 3^{6} 5^{2} \pi^{5}}$ of the genus-two amplitude. See appendix $\mathrm{E}$ for more details on the normalization of the genus-two amplitude.
} 


\subsubsection{Tree level}

In writing the expansion (4.50) we defined the shorthands

$$
\mathcal{B}_{\{n\}}^{\text {tree }}=2 \tilde{A}_{54}^{T} \cdot S_{0} \cdot M_{n} \cdot A_{45}, \quad \mathcal{B}_{\{3,3\}}^{\text {tree }}=2 \tilde{A}_{54}^{T} \cdot S_{0} \cdot M_{3}^{2} \cdot A_{45}
$$

in terms of the two-component vectors $\tilde{A}_{54}^{T}$ and $A_{45}$ of SYM tree-amplitudes and the momentum kernel $S_{0}$ of (4.28) and (4.29), respectively. Here $M_{n}$ are $2 \times 2$ matrices with entries composed of degree $n$ polynomials in kinematic invariants [34] generalizing (4.31), explicit results are available for download on [83]. For uniformity we set $M_{0}=\frac{1}{2} \mathrm{Id}$ and note that $\mathcal{B}_{\{0\}}^{\text {tree }}=\tilde{A}_{54}^{T} \cdot S_{0} \cdot A_{45}$ corresponds to the Kawai-Lewellen-Tye representation of the supergravity tree-level amplitude [84]. In addition,

$$
\mathcal{B}_{\left\{n^{\prime}\right\}}^{! \text {tree }}=2 \tilde{A}_{54}^{T} \cdot S_{0} \cdot M_{n}^{\prime} \cdot A_{45}
$$

where $M_{n}^{\prime}$ are similar $2 \times 2$ matrices with entries composed of degree $n$ polynomials in $s_{i j}$, but which only start to contribute at genus one [35]. The explicit form of $M_{7}^{\prime}$ can be found in the ancillary files of [35]. The notation $\mathcal{B}_{\left\{n^{\prime}\right\}}^{\text {!tre }}$ is meant to convey both the analogy with the matrices $M_{n}$ appearing at tree level, and to emphasize its absence at that level.

\subsubsection{Genus one}

Collecting the results from [35] and [8] we get, for 5 Type IIB gravitons

$$
\begin{array}{rlrl}
\left.\mathcal{B}_{\{3\}}^{\text {genus-1 }}\right|_{h^{5}}=-\left.\mathcal{B}_{\{3\}}^{\text {tree }}\right|_{h^{5}} & & \left(\mathcal{R}^{5} / k^{2}\right) \\
\left.\mathcal{B}_{\{5\}}^{\text {genus-1 }}\right|_{h^{5}}=\left.2 E_{2} \mathcal{B}_{\{5\}}^{\text {tree }}\right|_{h^{5}} & \left(D^{2} \mathcal{R}^{5}\right) \\
\left.\mathcal{B}_{\{3,3\}}^{\text {genus-1 }}\right|_{h^{5}}=\left.\left(5 E_{3}+\zeta_{3}\right) \mathcal{B}_{\{3,3\}}^{\text {tree }}\right|_{h^{5}} & \left(D^{4} \mathcal{R}^{5}\right) \\
\left.\mathcal{B}_{\{7\}}^{\text {genus-1 }}\right|_{h^{5}}=\left.\left(4 C_{2,1,1}+2 E_{2}^{2}-2 E_{4}\right) \mathcal{B}_{\{7\}}^{\text {tree }}\right|_{h^{5}} & \left(D^{6} \mathcal{R}^{5}\right) \\
\left.\mathcal{B}_{\left\{7^{\prime}\right\}}^{\text {genus-1 }}\right|_{h^{5}}=\left.\left(\frac{15}{4} C_{2,1,1}-\frac{25}{8} E_{2}^{2}+\frac{57}{8} E_{4}\right) \mathcal{B}_{\left\{7^{\prime}\right\}}^{\text {!tree }}\right|_{h^{5}} & & \left(D^{6} \mathcal{R}^{5}\right)^{\prime}
\end{array}
$$

and for 4 gravitons and 1 dilaton

$$
\begin{array}{rlrl}
\left.\mathcal{B}_{\{3\}}^{\text {genus }-1}\right|_{\phi h^{4}} & =\left.\frac{1}{3} \mathcal{B}_{\{3\}}^{\text {tree }}\right|_{\phi h^{4}} & \left(\phi \mathcal{R}^{4}\right) \\
\left.\mathcal{B}_{\{5\}}^{\text {genus }-1}\right|_{\phi h^{4}} & =\left.\frac{2}{5} E_{2} \mathcal{B}_{\{5\}}^{\text {tree }}\right|_{\phi h^{4}} & & \left(\phi D^{4} \mathcal{R}^{4}\right) \\
\left.\mathcal{B}_{\{3,3\}}^{\text {genus }-1}\right|_{\phi h^{4}} & =\left.\frac{1}{3}\left(5 E_{3}+\zeta_{3}\right) \mathcal{B}_{\{3,3\}}^{\text {tree }}\right|_{\phi h^{4}} & & \left(\phi D^{6} \mathcal{R}^{4}\right) \\
\left.\mathcal{B}_{\{7\}}^{\text {genus }-1}\right|_{\phi h^{4}} & =\left.\frac{3}{7}\left(4 C_{2,1,1}+2 E_{2}^{2}-2 E_{4}\right) \mathcal{B}_{\{7\}}^{\text {tree }}\right|_{\phi h^{4}} & & \left(\phi D^{8} \mathcal{R}^{4}\right) \\
\left.\mathcal{B}_{\left\{7^{\prime}\right\}}^{\text {genus }-1}\right|_{\phi h^{4}} & =\left.\left(\frac{43}{4} C_{2,1,1}-\frac{61}{8} E_{2}^{2}+\frac{93}{8} E_{4}\right) \mathcal{B}_{\left\{7^{\prime}\right\}}^{\text {!tree }}\right|_{\phi h^{4}} & & \left(\phi D^{8} \mathcal{R}^{4}\right)^{\prime}
\end{array}
$$

Here, $E_{k}$ is the standard non-holomorphic Eisenstein series, defined for $k \geq 2$ on a torus with modulus $\tau$ and momentum lattice $\Lambda=\mathbb{Z}+\tau \mathbb{Z}$ by,

$$
E_{k}(\tau)=\sum_{p \in \Lambda^{\natural}} \frac{\tau_{2}^{k}}{\pi^{k}|p|^{2 k}}
$$


where $\Lambda^{\natural}=\Lambda \backslash\{0\}$, while $C_{2,1,1}$ is the two-loop MGF [33] defined by,

$$
C_{2,1,1}(\tau)=\sum_{p_{1}, p_{2}, p_{3} \in \Lambda^{\prime}} \frac{\tau_{2}^{4} \delta\left(p_{1}+p_{2}+p_{3}\right)}{\pi^{4}\left|p_{1}\right|^{4}\left|p_{2}\right|^{2}\left|p_{3}\right|^{2}}
$$

In view of (4.55) and (4.56), the five-point genus-one amplitude (4.48) in Type IIB becomes

$$
\mathcal{A}_{(5)}^{\text {genus-1 }}=\delta(k)\left(\frac{\alpha^{\prime}}{2}\right) \frac{\kappa^{5}}{2^{5} \pi} \int_{\mathcal{M}_{1}} d \mu_{1} \times \begin{cases}\left.\mathcal{B}_{(5)}^{\text {genus-1 }}\right|_{h^{5}}: & \text { five gravitons } \\ \left.\mathcal{B}_{(5)}^{\text {genus-1 }}\right|_{\phi h^{4}}: & \text { four gravitons, one dilaton }\end{cases}
$$

where [35]

$$
\begin{aligned}
\left.\mathcal{B}_{(5)}^{\text {genus }-1}\right|_{h^{5}}= & -\left.\mathcal{B}_{\{3\}}^{\text {tree }}\right|_{h^{5}}+\left.2 E_{2} \mathcal{B}_{\{5\}}^{\text {tree }}\right|_{h^{5}}+\left.\left(5 E_{3}+\zeta_{3}\right) \mathcal{B}_{\{3,3\}}^{\text {tree }}\right|_{h^{5}} \\
& +\left.\left(4 C_{2,1,1}+2 E_{2}^{2}-2 E_{4}\right) \mathcal{B}_{\{7\}}^{\text {tree }}\right|_{h^{5}}+\left.\left(\frac{15}{4} C_{2,1,1}-\frac{25}{8} E_{2}^{2}+\frac{57}{8} E_{4}\right) \mathcal{B}_{\left\{7^{\prime}\right\}}^{! \text {tree }}\right|_{h^{5}}+\cdots \\
\left.\mathcal{B}_{(5)}^{\text {genus }-1}\right|_{\phi h^{4}}= & \left.\frac{1}{3} \mathcal{B}_{\{3\}}^{\text {tree }}\right|_{\phi h^{4}}+\left.\frac{2}{5} E_{2} \mathcal{B}_{\{5\}}^{\text {tree }}\right|_{\phi h^{4}}+\left.\frac{1}{3}\left(5 E_{3}+\zeta_{3}\right) \mathcal{B}_{\{3,3\}}^{\text {tree }}\right|_{\phi h^{4}} \\
& +\left.\frac{3}{7}\left(4 C_{2,1,1}+2 E_{2}^{2}-2 E_{4}\right) \mathcal{B}_{\{7\}}^{\text {tree }}\right|_{\phi h^{4}} \\
& +\left.\left(\frac{43}{4} C_{2,1,1}-\frac{61}{8} E_{2}^{2}+\frac{93}{8} E_{4}\right) \mathcal{B}_{\left\{7^{\prime}\right\}}^{\text {!tree }}\right|_{\phi h^{4}}+\cdots
\end{aligned}
$$

with terms of order $s_{i j}^{m \geq 8} A_{\mathrm{YM}} \tilde{A}_{\mathrm{YM}}$ in the ellipsis. Apart from the last term $\mathcal{B}_{\left\{7^{\prime}\right\}}^{\text {!tree }}$, these $\alpha^{\prime}$-corrections involve the same polynomial dependence on the $s_{i j}$ as the coefficients of $\zeta_{3}, \zeta_{5}, \zeta_{3}^{2}$ and $\zeta_{7}$ in the tree-level amplitude.

\subsubsection{Genus two}

Explicit pure spinor superspace component evaluations of the genus-two kinematic factors for 5 external Type IIB graviton states yield

$$
\begin{array}{llrl}
\left.\mathcal{B}_{\{5\}}^{\text {genus-2 }}\right|_{h^{5}} & =-\left.\mathcal{B}_{\{5\}}^{\text {tree }}\right|_{h^{5}} & & \left(D^{2} \mathcal{R}^{5}\right) \\
\left.\mathcal{B}_{\{3,3\}}^{\text {genus-2 }}\right|_{h^{5}}=\left.3 \varphi \mathcal{B}_{\{3,3\}}^{\text {tree }}\right|_{h^{5}} & & \left(D^{4} \mathcal{R}^{5}\right) \\
\left.\mathcal{B}_{\{7\}}^{\text {genus-2 }}\right|_{h^{5}}=-\left.\frac{1}{4} A_{1} \mathcal{B}_{\{7\}}^{\text {tree }}\right|_{h^{5}} & & \left(D^{6} \mathcal{R}^{5}\right) \\
\left.\mathcal{B}_{\left\{7^{\prime}\right\}}^{\text {genus-2 }}\right|_{h^{5}}=\left.A_{3} \mathcal{B}_{\left\{7^{\prime}\right\}}^{\text {!tree }}\right|_{h^{5}} & & \left(D^{6} \mathcal{R}^{5}\right)^{\prime}
\end{array}
$$

while for 4 gravitons and one dilaton,

$$
\begin{array}{rlrl}
\left.\mathcal{B}_{\{5\}}^{\text {genus }-2}\right|_{\phi h^{4}} & =\frac{3}{5} \mathcal{B}_{\{5\}}^{\text {tree }} & & \left(\phi D^{4} \mathcal{R}^{4}\right) \\
\left.\mathcal{B}_{\{3,3\}}^{\text {genus-2 }}\right|_{\phi h^{4}} & =-\varphi \mathcal{B}_{\{3,3\}}^{\text {tree }} & & \left(\phi D^{6} \mathcal{R}^{4}\right) \\
\left.\mathcal{B}_{\{7\}}^{\text {genus }-2}\right|_{\phi h^{4}} & =\frac{1}{28} A_{1} \mathcal{B}_{\{7\}}^{\text {tree }} & & \left(\phi D^{8} \mathcal{R}^{4}\right) \\
\left.\mathcal{B}_{\left\{7^{\prime}\right\}}^{\text {genus-2 }}\right|_{\phi h^{4}}=A_{4} \mathcal{B}_{\left\{7^{\prime}\right\}}^{\text {!tre }} & & \left(\phi D^{8} \mathcal{R}^{4}\right)^{\prime}
\end{array}
$$


where

$$
\begin{aligned}
& A_{3}=\frac{5}{64}\left(A_{1}-40 A_{2}-2 \mathcal{Z}_{5}\right) \\
& A_{4}=\frac{45}{64} A_{1}-\frac{17}{8} A_{2}-\frac{5}{32} \mathcal{Z}_{5}+\mathcal{Z}_{3}+2 \mathcal{Z}_{2}-6 \varphi^{2}
\end{aligned}
$$

Therefore, the five-point genus-two amplitude for Type IIB external states is given by,

$$
\mathcal{A}_{(5)}^{\text {genus-2 }}=\delta(k)\left(\frac{\alpha^{\prime}}{2}\right) \frac{\kappa^{5} e^{2 \lambda}}{2^{11} \pi^{5}} \int_{\mathcal{M}_{2}} d \mu_{2} \times \begin{cases}\left.\mathcal{B}_{(5)}^{\text {genus-2 }}\right|_{h^{5}}: & \text { five gravitons } \\ \left.\mathcal{B}_{(5)}^{\text {genus-2 }}\right|_{\phi h^{4}}: & \text { four gravitons, one dilaton }\end{cases}
$$

where

$$
\begin{aligned}
\left.\mathcal{B}_{(5)}^{\text {genus }-2}\right|_{h^{5}} & =-\left.\mathcal{B}_{\{5\}}^{\text {tree }}\right|_{h^{5}}+\left.3 \varphi \mathcal{B}_{\{3,3\}}^{\text {tree }}\right|_{h^{5}}-\left.\frac{1}{4} A_{1} \mathcal{B}_{\{7\}}^{\text {tree }}\right|_{h^{5}}+\left.A_{3} \mathcal{B}_{\left\{7^{\prime}\right\}}^{\text {!tree }}\right|_{h^{5}}+\cdots \\
\left.\mathcal{B}_{(5)}^{\text {genus-2 }}\right|_{\phi h^{4}} & =\left.\frac{3}{5} \mathcal{B}_{\{5\}}^{\text {tree }}\right|_{\phi h^{4}}-\left.\varphi \mathcal{B}_{\{3,3\}}^{\text {tree }}\right|_{\phi h^{4}}+\left.\frac{1}{28} A_{1} \mathcal{B}_{\{7\}}^{\text {tree }}\right|_{\phi h^{4}}+\left.A_{4} \mathcal{B}_{\left\{7^{\prime}\right\}}^{\text {tree }}\right|_{\phi h^{4}}+\cdots
\end{aligned}
$$

with terms of order $s_{i j}^{m \geq 8} A_{\mathrm{YM}} \tilde{A}_{\mathrm{YM}}$ in the ellipsis. The relative factor $-3 / 5$ between the ratios of the genus-two to tree-level amplitudes for the $h^{5}$ and $\phi h^{4}$ components at the order of $D^{2} \mathcal{R}^{5}$ agrees with the S-duality analysis of [8], while the factors $-1 / 3$ and $-1 / 7$ for the $D^{4} \mathcal{R}^{5}$ and $D^{6} \mathcal{R}^{5}$ interactions are new. In the next section we will explain these relative coefficients from the point of view of modular forms.

Note that the results of this section can be adapted to the entire massless Type IIB multiplet upon replacing $h^{5}$ or $\phi h^{4}$ by state configurations with the same $\mathrm{U}(1)_{R}$ charges.

\section{Consistency with supergravity and S-duality}

In this section, we shall check that our results for the low energy expansion of the genus-two five-point amplitudes are consistent with the structure of UV divergences in supergravity, in particular the absence of $\mathrm{U}(1)_{R}$-violating divergences in the supergravity limit, and with predictions from S-duality.

\subsection{R-symmetry violation and UV divergences in supergravity}

When the five external states are in a configuration which violate the supergravity $\mathrm{U}(1)_{R}$ symmetry the corresponding interaction is local [39]. For the specific case of $\phi h^{4}$ in Type IIB theory, the local interactions at different $\alpha^{\prime}$ orders can be written as $K_{\phi h^{4}} \sum_{m} a_{m} \mathcal{O}_{m}$ where $^{20}$

$$
K_{\phi h^{4}}=\left.\mathcal{B}_{\{3\}}^{\text {tree }}\right|_{\phi h^{4}}
$$

\footnotetext{
${ }^{20}$ In $D=10$ we have $\left.\mathcal{B}_{\{3\}}^{\text {tree }}\right|_{\phi h^{4}}=\frac{5}{8192}\left(t_{8} t_{8} \mathcal{R}^{4}-\frac{1}{8} \epsilon_{10}^{m} \tilde{\epsilon}_{10}^{m}\right)$ where $\epsilon_{10}^{m}=\epsilon_{10}\left(k_{2}, k_{3}, k_{4}, k_{5}, \varepsilon_{1}, \varepsilon_{2}, \varepsilon_{3}, \varepsilon_{4}, \varepsilon_{5}, m\right)$. However, this representation depends on the dimension of spacetime as there is a contraction between leftand right movers. That is why we chose the dimension-agnostic representation (5.1).
} 
$a_{m}$ are rational coefficients and $\mathcal{O}_{m}$ are symmetric polynomial in the kinematic invariants $s_{i j}$, which can be expressed in terms of the polynomials $P_{n}$ defined in (4.41). More specifically, the kinematic factors in (4.56) and (4.63) are related to (5.1) via

$$
\begin{aligned}
\left.\mathcal{B}_{\{5\}}^{\text {tree }}\right|_{\phi h^{4}} & =\frac{5}{12} \mathcal{O}_{2} K_{\phi h^{4}} & \left.\mathcal{B}_{\{3,3\}}^{\text {tree }}\right|_{\phi h^{4}} & =-\frac{1}{3} \mathcal{O}_{3} K_{\phi h^{4}} \\
\left.\mathcal{B}_{\{7\}}^{\text {tree }}\right|_{\phi h^{4}} & =\frac{7}{16} \mathcal{O}_{5,1} K_{\phi h^{4}} & \left.\mathcal{B}_{\left\{7^{\prime}\right\}}^{\text {!tree }}\right|_{\phi h^{4}} & =-\frac{1}{9} \mathcal{O}_{5,2} K_{\phi h^{4}}
\end{aligned}
$$

where [40]

$$
\mathcal{O}_{2}=P_{2}, \quad \mathcal{O}_{3}=P_{3}, \quad \mathcal{O}_{5,1}=P_{4}+\frac{1}{12} P_{2}^{2}, \quad \mathcal{O}_{5,2}=P_{4}-\frac{1}{4} P_{2}^{2} .
$$

We will in fact evaluate $R$-symmetry violating IIB amplitudes with a $D$-dimensional dilaton state with polarization

$$
\varepsilon_{1} \cdot \tilde{\varepsilon}_{1}=(D-2) \phi_{1}
$$

rather than the standard ten-dimensional dilaton. In those cases, the coefficients of the quantities in (5.2) in $\left.\mathcal{B}_{\{n\}}^{\text {genus-1 }}\right|_{\phi h^{4}}$ and $\left.\mathcal{B}_{\{n\}}^{\text {genus-2 }}\right|_{\phi h^{4}}$ become $D$-dependent and reduce to the expressions (4.56) and (4.63) if $D \rightarrow 10$.

\subsubsection{D-dimensional dilatons at genus two}

By evaluating the Type IIB components of the genus-two kinematic factors with four gravitons and one $D$-dimensional dilaton state (5.4), one arrives at

$\left.\mathcal{B}_{(5)}^{\text {genus }-2}\right|_{\phi h^{4}}=\frac{1}{10} K_{\phi h^{4}}\left[\frac{5}{6}(D-7) P_{2}+\frac{5}{3}(D-8) \varphi P_{3}+\frac{5}{32}(D-9) A_{1} \mathcal{O}_{5,1}+W_{D} \mathcal{O}_{5,2}+\mathcal{O}\left(s_{i j}^{5}\right)\right]$

where $K_{\phi h^{4}}=\mathcal{B}_{\{3\}}^{\text {tree }}$ and,

$$
W_{D}=(27-D) \frac{5}{36} A_{2}+(1-D) \frac{25}{288} A_{1}+(D-2)\left(-\frac{5}{18} \mathcal{Z}_{2}-\frac{5}{36} \mathcal{Z}_{3}+\frac{5}{6} \varphi^{2}\right)+\frac{25}{144} \mathcal{Z}_{5}
$$

These results allow for sharp tests of our expressions for the low energy expansion. Indeed, as discussed in $[48,49]$, the $\alpha^{\prime}$ expansion of the genus-two superstring amplitude must reproduce the logarithmic divergences of two-loop supergravity in various dimensions. For four-graviton scattering, UV divergences proportional to $D^{4} \mathcal{R}^{4}, D^{6} \mathcal{R}^{4}$ and $D^{8} \mathcal{R}^{4}$ arise in $D=7,8,9$, respectively [46], and the coefficient is precisely reproduced by the tropical limit of the string integrand [49]. At the five-point level, the UV divergences for 5 gravitons have not yet been computed in supergravity, but the UV divergences for 4 gravitons and one dilaton must certainly be absent, since supergravity amplitudes preserve R-symmetry. Indeed, from (5.5) it is apparent that the divergences proportional to $P_{2}, P_{3}$ and $\mathcal{O}_{5,1}$ in $D=7,8,9$ cancel as they should. This is not obvious, however, for the term proportional to $\mathcal{O}_{5,2}$, which is potentially divergent in $D=9$. In this dimension, the coefficient evaluates to

$$
W_{9}=\frac{25}{144}\left[-4 A_{1}+\frac{72}{5} A_{2}-\frac{56}{5} \mathcal{Z}_{2}-\frac{28}{5} \mathcal{Z}_{3}+\frac{168}{5} \varphi^{2}+\mathcal{Z}_{5}\right]
$$


Using the results in appendix C.3, one finds that in the tropical limit $V \rightarrow 0$,

$$
W_{9} \sim-\frac{5}{36} \frac{32 \pi^{2}}{V^{2}}\left[0-\frac{1}{63} A_{0,2}+\frac{25}{99} A_{1,1}-\frac{91}{120} A_{2,0}\right]+\mathcal{O}(V)
$$

As explained in ([49], (B.16)), the regularized integrals of the local modular forms $A_{0,2}, A_{1,1}, A_{2,0}$ (see section 5.3 of [32]) over the complex modulus $S$ parametrizing the Schwinger parameters $L_{1}, L_{2}, L_{3}$ at fixed discriminant $\operatorname{det} Y=L_{1} L_{2}+L_{2} L_{3}+L_{3} L_{1}$ vanish, so that the only UV divergence comes from the integral over $A_{0,0}=1$, whose coefficient vanishes in the combination (5.8).

\subsection{2 $D$-dimensional dilatons at genus one}

The genus-one analogue of (5.5) can be obtained by promoting the results of ([35], section 5.3) to a $D$-dimensional dilaton state,

$$
\begin{aligned}
\left.\mathcal{B}_{(5)}^{\text {genus }-1}\right|_{\phi h^{4}}= & K_{\phi h^{4}}\left[\frac{1}{6}(D-8)+\frac{E_{2}}{12}(D-12) P_{2}-\frac{\left(5 E_{3}+\zeta_{3}\right)}{36}(D-14) P_{3}\right. \\
& \left.+\frac{1}{16}\left(2 C_{2,1,1}+E_{2}^{2}-E_{4}\right)(D-16) \mathcal{O}_{5,1}+W_{D}^{\prime} \mathcal{O}_{5,2}+\mathcal{O}\left(s_{i j}^{5}\right)\right]
\end{aligned}
$$

where

$$
W_{D}^{\prime}=\left(\frac{2}{9}+\frac{7 D}{72}\right) C_{2,1,1}+\left(\frac{2}{3}+\frac{D}{16}\right) E_{4}-\left(\frac{2}{9}+\frac{D}{16}\right) E_{2}^{2}
$$

The contributions of $P_{2}, P_{3}$ and $\mathcal{O}_{5,1}$ to (5.9) correspond to one-loop UV divergences proportional to $D^{4} \mathcal{R}^{4}, D^{6} \mathcal{R}^{4}$ and $D^{8} \mathcal{R}^{4}$ which occur in $D=12,14$ and $D=16$, respectively. Again, the R-symmetry violation by these UV divergences is prevented by the prefactors $(D-12),(D-14)$ and $(D-16)$ in $(5.9)$, and the coefficient $(5.10)$ of $\mathcal{O}_{5,2}$ requires closer inspection in the critical dimension $D=16$ :

$$
\begin{aligned}
W_{16}^{\prime} & =\frac{1}{9}\left(16 C_{2,1,1}+15 E_{4}-11 E_{2}^{2}\right) \\
& =-\frac{2 \pi \tau_{2} \zeta_{3}}{135}+\frac{20 \zeta_{5}}{27 \pi \tau_{2}}-\frac{5 \zeta_{3}^{2}}{3 \pi^{2} \tau_{2}^{2}}+\frac{49 \zeta_{7}}{24 \pi^{3} \tau_{2}^{3}}+\mathcal{O}\left(e^{-2 \pi \tau_{2}}\right)
\end{aligned}
$$

In passing to the second line, we have inserted the asymptotics of the modular graph functions around the cusp [27] which is captured by Laurent polynomials in $\tau_{2}$. The order of $\tau_{2}^{4}$ which is present in the individual $C_{2,1,1}, E_{2}^{2}$ and $E_{4}$ drops out from the particular combination in $W_{16}^{\prime}$ and signals the absence of a 16-dimensional UV divergence in supergravity as expected.

Note that the classes of multiple zeta values in the Laurent expansion of modular graph functions as in (5.11) are under active investigation in both the physics and mathematics literature [33, 70, 85-88]. By comparing with the multiple zeta values in the tree-level effective action of the Type IIB and IIA theories [34], one can associate the leftover terms in (5.11) with UV divergences due to loop diagrams with insertions of $D^{2 k} \mathcal{R}^{n}$ operators with $n \geq 4[48,49]$. 


\section{$5.2 \quad$ S-duality analysis}

According to the standard S-duality conjecture in Type IIB string theory, the low energy effective action must be invariant under the action of $\mathrm{SL}(2, \mathbb{Z})$. In Einstein frame, $\operatorname{SL}(2, \mathbb{Z})$ acts by fractional linear transformations on the axion-dilaton field $\tau=a+i / g_{s}^{2}$, and by $\mathrm{U}(1)_{R}$ rotations on the other fields, leaving the metric invariant. Thus, the coefficients of effective interactions violating $\mathrm{U}(1)_{R}$ symmetry by $2 q$ units must transform with modular weight $(q,-q)$ under S-duality. Typically, these interactions are related to $\mathrm{U}(1)_{R}$-preserving interactions by non-linear supersymmetry, so that their coefficients are obtained by acting repeatedly with a covariant derivative operator $\mathcal{D}=\tau_{2} \partial_{\tau}-\frac{i w}{2}$, which maps modular forms of weight $(w, \bar{w})$ to modular forms of weight $(w+1, \bar{w}-1)$. An example of this is the dilatino vertex $\Lambda^{16}$, which violates $\mathrm{U}(1)_{R}$ by 24 units and is related to the $\mathcal{R}^{4}$ coupling by acting with $\mathcal{D}^{12}[89]$.

At the four-point level, the amplitudes must conserve the $\mathrm{U}(1)_{R}$ charge, and are all related to four-graviton scattering by supersymmetry. The expansion of the analytic part of the four-graviton all-genus amplitude in Einstein frame takes the form [25],

$$
\left.\mathcal{A}_{(4)}\right|_{\text {analytic }}=\left|t_{8}\left(f_{1}, f_{2}, f_{3}, f_{4}\right)\right|^{2}\left[\frac{g_{s}^{-2}}{\sigma_{3}}+\mathcal{E}_{(0,0)}+\sigma_{2} \mathcal{E}_{(1,0)}+\sigma_{3} \mathcal{E}_{(0,1)}+\frac{\sigma_{2}^{2}}{2} \mathcal{E}_{(2,0)}+\mathcal{O}\left(s_{i j}^{5}\right)\right]
$$

At each order, the coefficient $\mathcal{E}_{(p, q)}$ of the term $\frac{\sigma_{2}^{p} \sigma_{3}^{q}}{p ! q !}$ (where $\sigma_{k}$ are the symmetric polynomials in (4.9)) must be a modular function of $\tau$ under the action of S-duality. The coefficients $\mathcal{E}_{(0,0)}$ and $\mathcal{E}_{(1,0)}$ of the first interactions $\mathcal{R}^{4}$ and $D^{4} \mathcal{R}^{4}$ beyond supergravity are well-known to be captured by the non-holomorphic Eisenstein series $E_{3 / 2}$ and $E_{5 / 2}$ defined in (4.57) $[16,18]$, whereas the next term $D^{6} \mathcal{R}^{4}$ involves a more complicated type of automorphic function $\mathcal{E}_{(0,1)}$ constructed in $[19,20]$.

For five-particle scattering, the $\mathrm{U}(1)_{R}$ symmetry is violated by at most 2 units, e.g in the scattering of one dilaton and 4 gravitons. We expect that the 5 -graviton interaction $D^{2} \mathcal{R}^{5}$ is related by non-linear supersymmetry [35] to the 4 -graviton $D^{4} \mathcal{R}^{4}$ interaction, governed by the automorphic form $\mathcal{E}_{(1,0)}$ with weak coupling expansion,

$$
\mathcal{E}_{(1,0)}=2 \zeta_{5} e^{-5 \phi / 2}+0+\frac{8}{3} \zeta_{4} e^{3 \phi / 2}+\cdots
$$

corresponding to the tree-level, vanishing genus-one and non-vanishing genus-two contributions, plus instanton corrections indicated by the dots. By linear supersymmetry, it follows that the $\phi D^{4} \mathcal{R}^{4}$ interaction between one dilaton and 4 gravitons at the same order in the derivative expansion should be controlled by,

$$
\mathcal{D} \mathcal{E}_{(1,0)} \propto-5 \zeta_{5} e^{-5 \phi / 2}+0+4 \zeta_{4} e^{3 \phi / 2}+\cdots
$$

where we use the fact that $\mathcal{D}$ maps $e^{q \phi} \rightarrow q e^{q \phi}$. This predicts that the ratio of the genus-two and tree-level contributions to $\phi D^{4} \mathcal{R}^{4}$ is modified by a factor $-3 / 5$ compared to the ratio of the genus-two and tree-level contributions to the $D^{2} \mathcal{R}^{5}$ coupling, in perfect agreement with (4.62), as noted already in [8]. 
By the same logic, the $D^{4} \mathcal{R}^{5}$ coupling is expected to be related by non-linear supersymmetry to the $D^{6} \mathcal{R}^{4}$ coupling, governed by the automorphic function $\mathcal{E}_{(0,1)}[19,20]$ with weak coupling expansion

$$
\mathcal{E}_{(0,1)}=4 \zeta_{3}^{2} e^{-3 \phi}+8 \zeta_{2} \zeta_{3} e^{-\phi}+\frac{48}{5} \zeta_{2}^{2} e^{\phi}+\frac{8}{9} \zeta_{6} e^{3 \phi}+\ldots
$$

corresponding to tree-level up to genus-three contributions, plus instanton and antiinstanton corrections indicated by the dots. By linear supersymmetry, it follows that the $\phi D^{6} \mathcal{R}^{4}$ interaction between one dilaton and 4 gravitons should be controlled by,

$$
\mathcal{D} \mathcal{E}_{(0,1)} \propto-12 \zeta_{3}^{2} e^{-3 \phi}-8 \zeta_{2} \zeta_{3} e^{-\phi}+\frac{48}{5} \zeta_{2}^{2} e^{\phi}+\frac{8}{3} \zeta_{6} e^{3 \phi}+\ldots
$$

This predicts that the ratio of the genus- $(1,2,3)$ to the tree-level contributions to $\phi D^{6} \mathcal{R}^{4}$ is modified by factors $(1 / 3,-1 / 3,-1)$ compared to the ratio of the genus- $(1,2,3)$ to the tree-level contributions to the $D^{4} \mathcal{R}^{5}$ coupling. At genus one, the factor $1 / 3$ was checked in ([35], section 5.3), and at genus two, the factor $-1 / 3$ is again in perfect agreement with (4.63).

Assuming that the coefficient of the $D^{6} \mathcal{R}^{5}$ interaction, related to $D^{8} \mathcal{R}^{4}$ by non-linear supersymmetry, has the weak coupling expansion (where the dots now stand for additional perturbative and non-perturbative corrections),

$$
\mathcal{E}_{(2,0)}=a_{0} e^{-\frac{7}{2} \phi}+a_{1} e^{-\frac{3}{2} \phi}+a_{2} e^{\frac{1}{2} \phi}+\cdots
$$

the $\phi D^{8} \mathcal{R}^{4}$ interaction following from linear supersymmetry is then accompanied by,

$$
\mathcal{D} \mathcal{E}_{(2,0)} \propto-\frac{7}{2} a_{0} e^{-\frac{7}{2} \phi}-\frac{3}{2} a_{1} e^{-\frac{3}{2} \phi}+\frac{1}{2} a_{2} e^{\frac{1}{2} \phi}+\cdots
$$

predicting a factor $-1 / 7$ between the ratios of the genus-two and tree-level contributions to $D^{6} \mathcal{R}^{5}$ and $\phi D^{8} \mathcal{R}^{4}$, respectively. This is indeed in agreement with (4.67) and (4.68).

Note that the genus-two interactions $\left(D^{6} \mathcal{R}^{5}\right)^{\prime}$ and $\left(\phi D^{8} \mathcal{R}^{4}\right)^{\prime}$ proportional to $A_{3}$ and $A_{4}$ in (4.64) and (4.65) do not have any corresponding interactions at tree level since the tree-level coefficient of $\mathcal{B}_{\left\{7^{\prime}\right\}}^{\text {!tree }}$ vanishes. Instead, we should consider the ratio of the genus-two and genus-one contributions. From $[35],{ }^{21}$ we find that the ratio of the oneloop contributions to $\phi h^{4}$ compared to $h^{5}$ is $9 / 5$. Defining, in analogy with (4.11), the regularization-dependent coefficients $c_{3}, c_{4}$ by,

$$
\int_{\mathcal{M}_{2}(\Lambda)} d \mu_{2} A_{3}=c_{3}(\Lambda) \mathrm{Vol}_{2}, \quad \int_{\mathcal{M}_{2}(\Lambda)} d \mu_{2} A_{4}=c_{4}(\Lambda) \mathrm{Vol}_{2}
$$

we predict that $c_{3} / c_{4}=-3 /(9 / 5)=-5 / 3$. Indeed, using the results in appendix $\mathrm{C}$, we find that the $\mathcal{O}\left(t^{2}\right)$ coefficients in the minimal non-separating degeneration for $A_{3}$ and $A_{4}$, responsible for logarithmic divergences in $D=10$, are in the ratio $-5 / 3$. In particular, the combination $c_{3}+\frac{5}{3} c_{4}$ is infrared finite in $D=10$.

\footnotetext{
${ }^{21}$ In ([35], eq. (5.4)), the statements $\int_{\mathcal{M}_{1}} d \mu_{1}\left(D_{4}, D_{2}^{2}, D_{211}\right)=0$ turn out to be incorrect; instead, one can use $\int_{\mathcal{M}_{1}} d \mu_{1} D_{1111}=0, \int_{\mathcal{M}_{1}} d \mu_{1} D_{211}=-\frac{1}{2} \int_{\mathcal{M}_{1}} d \mu_{1} D_{2}^{2}$ and the identity $D_{4}=24 D_{211}+3 D_{2}^{2}-18 D_{1111}$ from [42] to express all integrals in terms of a single one, leading to $\Xi_{7^{\prime}}=-\frac{5 \pi}{2} \int_{\mathcal{M}_{1}} d \mu_{1} D_{2}^{2}$ and $\hat{\Xi}_{7^{\prime}}=-$ $\frac{9 \pi}{2} \int_{\mathcal{M}_{1}} d \mu_{1} D_{2}^{2}$
} 


\section{Acknowledgments}

The research of ED is supported in part by NSF grant PHY-19-14412. BP and OS are grateful to UCLA and the Mani Bhaumik Institute for kind hospitality and creating a stimulating atmosphere during initial stages of this work. CRM is supported by a University Research Fellowship from the Royal Society. OS is grateful to AEI Potsdam for kind hospitality during final stages of this work. Moreover, OS is supported by the European Research Council under ERC-STG-804286 UNISCAMP.

\section{A Functions on Riemann surfaces}

In this appendix, we shall collect definitions, notations, and conventions for holomorphic forms, the period matrix, bi-holomorphic forms, the Arakelov Green function, and present some of the basic formulas needed in this paper for integrals involving these quantities.

\section{A.1 Convention for forms}

Throughout, we shall follow the conventions of [12] and display only the coefficient functions of differentials on a Riemann surface $\Sigma$ in a system of local complex coordinates $(z, \bar{z})$ on $\Sigma$. In this convention, a $(1,0)$ form $\omega d z$ will be referred to as $\omega$ and its integral along a curve $\mathcal{C}$ will be abbreviated $\int_{\mathcal{C}} d z \omega \rightarrow \int_{\mathcal{C}} \omega$, while a $(1,1)$-form $v d z \wedge d \bar{z}$ will be referred to as $v$ and its integral on $\Sigma$ will be abbreviated $\int_{\Sigma} d z \wedge d \bar{z} v \rightarrow \int_{\Sigma} v$. In the particular case of interest here the $(1,1)$ form may be the result of a wedge product between a $(1,0)$ form $\omega d z$ and a $(0,1)$ form $\bar{\psi} d \bar{z}$, in which case the convention is $\int_{\Sigma} \omega d z \wedge \bar{\psi} d \bar{z} \rightarrow \int_{\Sigma} \omega \bar{\psi}=\int_{\Sigma} \bar{\psi} \omega$ because the component functions $\omega$ and $\bar{\psi}$ commute with one another. We shall also use the abbreviation $\int_{u}=\int_{\Sigma_{u}}$ to indicate the integration over $\Sigma$ in the variable $u$.

\section{A.2 Holomorphic 1-forms and the period matrix}

We choose a canonical basis of $\mathfrak{A}_{I}$ and $\mathfrak{B}_{I}$ cycles in $H_{1}(\Sigma, \mathbb{Z})$ for which the intersection pairing $\mathfrak{J}$ takes the form of the standard symplectic matrix, $\mathfrak{J}\left(\mathfrak{A}_{I}, \mathfrak{A}_{J}\right)=\mathfrak{J}\left(\mathfrak{B}_{I}, \mathfrak{B}_{J}\right)=0$ and $\mathfrak{J}\left(\mathfrak{A}_{I}, \mathfrak{B}_{J}\right)=\delta_{I J}$ for $I, J=1,2$. A canonical basis of holomorphic Abelian differentials $\omega_{I}$ for $H^{(1,0)}(\Sigma)$ may be normalized on $\mathfrak{A}$-cycles, and we have,

$$
\oint_{\mathfrak{A}_{I}} \omega_{J}=\delta_{I J} \quad \oint_{\mathfrak{B}_{I}} \omega_{J}=\Omega_{I J}
$$

By the Riemann relations, the period matrix $\Omega$ is symmetric, and has positive definite imaginary part $Y$ as a result of the following pairing relation,

$$
\int_{\Sigma} \omega_{I} \overline{\omega_{J}}=-2 i Y_{I J} \quad Y=\operatorname{Im} \Omega>0
$$

The Siegel upper half space $\mathcal{H}_{2}$ may be defined as the space of all $2 \times 2$ complex-valued symmetric matrices whose imaginary part is positive definite. Alternatively, a more geometrical definition is $\mathcal{H}_{2}=\mathrm{Sp}(4, \mathbb{R}) /(\mathrm{SU}(2) \times \mathrm{U}(1))$. The presence of the $\mathrm{U}(1)$ factor 
implies that $\mathcal{H}_{2}$ is a Kähler manifold and its $\operatorname{Sp}(4, \mathbb{R})$-invariant Kähler metric is given as follows, ${ }^{22}$

$$
d s^{2}=Y^{I K} Y^{J L} d \Omega_{I J} d \bar{\Omega}_{K L}
$$

where $\left(Y^{-1}\right)^{I J}=Y^{I J}$ are the components of the inverse of the matrix $Y$. The moduli space $\mathcal{M}_{2}$ may be identified with $\mathcal{M}_{2}=\operatorname{Sp}(4, \mathbb{Z}) \backslash \mathcal{H}_{2}$ provided we remove from $\mathcal{H}_{2}$ all elements which correspond to disconnected surfaces.

The Jacobian variety $J(\Sigma)=\mathbb{C}^{2} /\left(\mathbb{Z}^{2}+\Omega \mathbb{Z}^{2}\right)$ supports the canonical Kähler form $K$,

$$
K=\frac{i}{2} Y^{I J} d \zeta_{I} d \bar{\zeta}_{J}
$$

where $\zeta_{I}$ are local complex coordinates on the flat torus $J(\Sigma)$. The form $\kappa$ is the pull-back of $K$ from $J(\Sigma)$ to $\Sigma$ under the Abel-Jacobi map and, for a compact Riemann surface $\Sigma$, may be normalized to unit volume,

$$
\kappa=\frac{i}{4} Y^{I J} \omega_{I} \overline{\omega_{J}} \quad \int_{\Sigma} \kappa=1
$$

The form $\kappa$ is conformal invariant as it is constructed solely out of the conformal invariant Abelian differentials.

\section{A.3 The bi-holomorphic forms $\Delta$ and $\nu$}

We define the bi-holomorphic form $\Delta$ by the anti-symmetric combination of $(1,0)$ forms,

$$
\Delta(x, y)=\varepsilon^{I J} \omega_{I}(x) \omega_{J}(y)=-\Delta(y, x)
$$

where $\varepsilon^{I J}=-\varepsilon^{J I}$ and $\varepsilon^{12}=1$. Moreover, the ubiquitous anti-hermitian combination $\nu(x, y)$ of $(1,0)$ and $(0,1)$ forms is defined in $(3.11)$. We shall list useful relations between the forms $\kappa, \Delta$ and $\nu$ in the remainder of this subsection, and give useful integral relations between these forms in the next subsection.

The identity $\varepsilon^{I J} \varepsilon^{K L}+\varepsilon^{I K} \varepsilon^{L J}+\varepsilon^{I L} \varepsilon^{J K}=0$ implies,

$$
\begin{aligned}
\omega_{I}(x) \Delta(y, z)+\omega_{I}(y) \Delta(z, x)+\omega_{I}(z) \Delta(x, y) & =0 \\
\Delta(w, x) \Delta(y, z)+\Delta(w, y) \Delta(z, x)+\Delta(w, z) \Delta(x, y) & =0
\end{aligned}
$$

The form $\nu$ obeys simple relations with $\Delta$ and $\kappa$,

$$
\begin{aligned}
& \Delta(x, y) \overline{\Delta(w, z)}=4(\operatorname{det} Y)(\nu(x, z) \nu(y, w)-\nu(x, w) \nu(y, z)) \\
& \Delta(x, y) \overline{\Delta(y, z)}=4(\operatorname{det} Y)(2 \nu(x, z) \kappa(y)-\nu(x, y) \nu(y, z)) \\
& \Delta(x, y) \overline{\Delta(y, x)}=4(\operatorname{det} Y)(4 \kappa(x) \kappa(y)-\nu(x, y) \nu(y, x))
\end{aligned}
$$

where the second equation follows from the first by setting $w=y$ and the third follows from the second by setting $z=x$. The following formula is being used to establish these results,

$$
\varepsilon^{I J} \varepsilon^{K L}=(\operatorname{det} Y)\left(Y^{I K} Y^{J L}-Y^{I L} Y^{J K}\right)
$$

\footnotetext{
${ }^{22}$ Throughout, summation over pairs of repeated upper and lower indices will be implied.
} 
The cyclic identities (A.7) imply further relations between $\kappa, \nu$ and $\Delta$,

$$
\begin{aligned}
\nu(x, w) \Delta(y, z)+\nu(y, w) \Delta(z, x)+\nu(z, w) \Delta(x, y) & =0 \\
2 \kappa(x) \Delta(y, z)+\nu(y, x) \Delta(z, x)+\nu(z, x) \Delta(x, y) & =0
\end{aligned}
$$

Note that $\kappa$ and $\nu$ may be defined for arbitrary genus, but $\Delta$ exists only for genus two.

\section{A.4 Some useful integrals}

Useful integrals involving $\Delta$ are as follows,

$$
\begin{gathered}
\int_{u} \omega_{I}(u) \overline{\Delta(u, y)}=-2 i Y_{I J} \varepsilon^{J K} \bar{\omega}_{K}(y) \\
\int_{u} \Delta(x, u) \overline{\Delta(u, y)}=4(\operatorname{det} Y) \nu(x, y)
\end{gathered}
$$

Useful integrals involving $\nu$ and $\Delta$ are as follows,

$$
\begin{gathered}
\int_{u} \nu(x, u) \omega_{I}(u)=\omega_{I}(x) \\
\int_{u} \nu(x, u) \nu(u, y)=\nu(x, y) \\
\int_{u} \nu(x, u) \Delta(u, y)=\Delta(x, y)
\end{gathered}
$$

The following double integrals will also come in handy,

$$
\begin{aligned}
\int_{u} \int_{v} \nu(u, v) \nu(v, u) & =2 \\
\int_{u} \int_{v} \Delta(u, v) \overline{\Delta(v, u)} & =8 \operatorname{det} Y \\
\int_{u} \int_{v} \Delta(x, u) \overline{\Delta(u, v)} \Delta(v, y) & =4(\operatorname{det} Y) \Delta(x, y)
\end{aligned}
$$

They may all be derived by making use of (A.2) to carry out the integrals, and then using algebraic relations between $Y$ and $\varepsilon$ to express the result in simplified form.

\section{A.5 The Arakelov Green function}

The Arakelov Green function $\mathcal{G}(x, y)$ is a real-valued symmetric function on $\Sigma \times \Sigma$ which provides an inverse to the scalar Laplace operator on $\Sigma$ with the canonical metric associated with $\kappa$, on the space of functions orthogonal to constants. In terms of local complex coordinates $(z, \bar{z})$ and the convention stated in subsection A.1, we have,

$$
\begin{array}{ll}
\partial_{z} \partial_{\bar{z}} \mathcal{G}(z, y)=-\pi \delta^{(2)}(z, y)-2 \pi i \kappa(z) & \int_{\Sigma} \kappa(z) \mathcal{G}(z, y)=0 \\
\partial_{z} \partial_{\bar{y}} \mathcal{G}(z, y)=\pi \delta^{(2)}(z, y)+2 \pi i \nu(z, y) &
\end{array}
$$

where $\delta^{(2)}(z, y)$ is the coordinate Dirac $\delta$-function normalized by,

$$
\frac{i}{2} \int_{\Sigma} d z \wedge d \bar{z} \delta^{(2)}(z, y)=1
$$


Note that the right side of the first equation in (A.14) integrates to zero in $z$ against constants, while the right side of the second equation integrates to zero in $y$ against the holomorphic forms $\omega_{I}(y)$ and in $z$ against the anti-holomorphic forms $\bar{\omega}_{I}(z)$. An explicit expression for $\mathcal{G}$ may be obtained by relating it to the Green function $G$ which is often used in string theory, as reviewed for example in [31].

\section{A.6 Reducing integrals of Arakelov Green functions}

Beyond the basic integrals $\mathcal{Z}_{1}, \ldots, \mathcal{Z}_{5}$ defined in (3.14), in expanding the five-point amplitude up to order $D^{6} \mathcal{R}^{5}$ we encounter various other integrals which can be easily reduced to the ones above, along with the square of the Kawazumi-Zhang invariant, ${ }^{23}$

$$
\begin{aligned}
\int_{\Sigma^{3}} \kappa(1) \nu(2,4) \nu(4,2) \mathcal{G}(1,2) \mathcal{G}(1,4) & =-\frac{1}{4} \mathcal{Z}_{2} \\
\int_{\Sigma^{3}} \nu(1,2) \nu(2,4) \nu(4,1) \mathcal{G}(1,2) \mathcal{G}(1,4) & =-\frac{1}{4} \mathcal{Z}_{2} \\
\int_{\Sigma^{4}} \nu(1,2) \nu(2,3) \nu(3,4) \nu(4,1) \mathcal{G}(1,2) \mathcal{G}(3,4) & =\frac{1}{2} \varphi^{2} \\
\int_{\Sigma^{4}} \nu(1,2) \nu(2,3) \nu(3,4) \nu(4,1) \mathcal{G}(1,3) \mathcal{G}(2,4) & =\frac{1}{2} \mathcal{Z}_{3}-\frac{1}{2} \varphi^{2} \\
\int_{\Sigma^{4}} \frac{\Delta(1,2) \overline{\Delta(2,3)} \Delta(3,4) \overline{\Delta(4,1)}}{(\operatorname{det} Y)^{2}} \mathcal{G}(1,3) \mathcal{G}(2,4) & =8 \mathcal{Z}_{3}-8 \varphi^{2} \\
\int_{\Sigma^{5}} \frac{\mathcal{G}(1,4)}{(\operatorname{det} Y)^{2}} \partial_{1} \mathcal{G}(1,2) \bar{\partial}_{1} \mathcal{G}(1,3) \Delta(2,4) \Delta(3,5) \overline{\Delta(2,5)} \overline{\Delta(3,4)} & =i \pi \mathcal{Z}_{5} \\
\int_{\Sigma^{5}} \frac{\mathcal{G}(1,3)}{(\operatorname{det} Y)^{2}} \partial_{1} \mathcal{G}(1,2) \bar{\partial}_{1} \mathcal{G}(1,4) \Delta(2,4) \Delta(3,5) \overline{\Delta(2,5)} \overline{\Delta(3,4)} & =-i \pi \mathcal{Z}_{5}
\end{aligned}
$$

The first two lines of (A.16) follow from using the last line of (A.8), and the last line of (A.10) on the combination $\kappa(1) \Delta(2,4)$, respectively, in the definition (3.14) of $\mathcal{Z}_{2}$. To derive the third line of (A.16), we use the second line of (A.7) on the product $\Delta(1,3) \Delta(2,4)$ and its complex conjugate in the third line of (3.14), cancel the $\mathcal{Z}_{3}$ contribution, and express the remainder in terms of $\varphi$. To derive the last line of (A.16), we use the second line of (A.7) on the product $\Delta(1,3) \Delta(2,4)$ but not on its complex conjugate in (3.14), and recast one of the integrals in terms of $\varphi^{2}$, giving the desired integral.

\section{B Expanding the integrals}

In this appendix, we spell out intermediate steps in obtaining the $\alpha^{\prime}$ expansion of the fivepoint integrals $J_{1, i}, F_{j}, G_{j}, H_{j}$ defined in (3.3) to (3.6). The integrals $J_{1, i}, G_{j}, H_{j}$ admit convergent Taylor series expansions at $s_{i j}=0$, while each $F_{j}$ has a simple poles in $s_{12}$. Since the integrals $H_{j}$ and $G_{j}$ will be needed only to order $s_{i j}$ they are the simplest and will be carried out first. The integrals $J_{1, i}$ will be needed to order $s_{i j}^{2}$ and are carried out next, finishing with $F_{j}$ which may be expressed in terms of the integrals $J_{1, i}$ and $G_{j}$.

\footnotetext{
${ }^{23}$ The second, third, and fourth integrals were denoted by $\mathcal{B}_{5}^{(2,0)}, \mathcal{B}_{6}^{(2,0)}, \mathcal{B}_{7}^{(2,0)}$ in [41].
} 


\section{B.1 The $H$-integrals}

The $H$-integrals defined in (3.6) have a convergent Taylor series expansion at $s_{i j}=0$. The contributions of order $\mathcal{O}\left(s_{i j}^{0}\right)$ clearly vanish. For the contributions of order $\mathcal{O}\left(s_{i j}\right)$, only the term proportional to $s_{13}$ is non-vanishing, so that we get,

$$
H_{j}=\frac{s_{13}}{i \pi} \int_{\Sigma^{5}} \partial_{1} \mathcal{G}(1,2) \bar{\partial}_{3} \mathcal{G}(3,4) \mathcal{G}(1,3) \frac{\Xi_{j}}{(\operatorname{det} Y)^{2}}+\mathcal{O}\left(s_{i j}^{2}\right)
$$

where $\Xi_{j}$ is a shorthand for the combination of $\Delta$ and $\bar{\Delta}$ in (3.6). Upon integrations by parts, the $\partial_{1}$ and $\bar{\partial}_{3}$ differentials can be made to both act on $\mathcal{G}(1,3)$ which gives

$$
H_{j}=s_{13} \int_{\Sigma^{4}} \mathcal{G}(1,2) \mathcal{G}(3,4)\left[-i \delta^{(2)}\left(z_{1}, z_{3}\right)+2 \nu(1,3)\right] \int_{z_{5}} \frac{\Xi_{j}}{(\operatorname{det} Y)^{2}}+\mathcal{O}\left(s_{i j}^{2}\right)
$$

based on (A.14). With the $\Delta$ and $\bar{\Delta}$ in (3.6), integration over point 5 yields,

$$
\begin{aligned}
& \int_{z_{5}} \frac{\Xi_{1}}{(\operatorname{det} Y)^{2}}=-4 \nu(3,1) \frac{\Delta(2,4) \overline{\Delta(2,4)}}{\operatorname{det} Y} \rightarrow-16 \nu(2,4) \nu(4,2) \nu(3,1) \\
& \int_{z_{5}} \frac{\Xi_{2}}{(\operatorname{det} Y)^{2}}=-4 \nu(4,2) \frac{\Delta(2,3) \overline{\Delta(1,4)}}{\operatorname{det} Y} \rightarrow 16 \nu(4,2)[\nu(2,1) \nu(3,4)-\nu(2,4) \nu(3,1)] \\
& \int_{z_{5}} \frac{\Xi_{3}}{(\operatorname{det} Y)^{2}}=-4 \nu(4,1) \frac{\Delta(2,3) \overline{\Delta(2,4)}}{\operatorname{det} Y} \rightarrow-16 \nu(3,2) \nu(2,4) \nu(4,1) \\
& \int_{z_{5}} \frac{\Xi_{4}}{(\operatorname{det} Y)^{2}}=-4 \nu(3,2) \frac{\Delta(2,4) \overline{\Delta(1,4)}}{\operatorname{det} Y} \rightarrow-16 \nu(3,2) \nu(2,4) \nu(4,1)
\end{aligned}
$$

where terms involving $\kappa(j)$ have been dropped in the step marked by the arrow since they integrate to zero in presence of $\mathcal{G}(1,2) \mathcal{G}(3,4)$. Decomposing the remaining integrals via (A.16), we find the results in (3.22).

\section{B.2 The $G$-integrals}

The $G$-integrals defined in (3.5) also admit a convergent Taylor series expansion at $s_{i j}=0$. Integrating by parts the factor $\bar{\partial}_{1} \mathcal{G}(1,3)$ one readily sees that they vanish at leading order in $s_{i j}$, except for $G_{2}$ which turns out to be proportional to the Kawazumi-Zhang invariant:

$$
\begin{aligned}
G_{2} & =-\frac{1}{i \pi} \int_{\Sigma^{5}} \frac{\partial_{1} \bar{\partial}_{1} \mathcal{G}(1,2)}{(\operatorname{det} Y)^{2}} \mathcal{G}(1,3) \Delta(2,4) \Delta(3,5) \overline{\Delta(2,5)} \overline{\Delta(3,4)}+\mathcal{O}\left(s_{i j}\right) \\
& =-32 \varphi+\mathcal{O}\left(s_{i j}\right)
\end{aligned}
$$

Similar manipulations may be used to obtain the results (3.21) to order $s_{i j}^{2}$ included in terms of the functions $\varphi, \mathcal{Z}_{1}, \mathcal{Z}_{2}, \mathcal{Z}_{3}, \mathcal{Z}_{4}$, plus the additional integral $\mathcal{Z}_{5}$ defined in (3.14).

\section{B.3 The $J$-integrals}

The $J$-integrals were defined in (3.3). It is straightforward to evaluate their leading $s_{i j}$ contributions $J_{r, s}^{(0)}$,

$$
J_{1,1}^{(0)}=128 \quad J_{1,2}^{(0)}=32 \quad J_{1,3}^{(0)}=-64
$$




\section{B.3.1 First order in $s$}

Evaluating the first order corrections, given by the sum over $s_{i j} \mathcal{G}(i, j)$ for $i<j$, for $J_{1,1}$ we see that the contributions where $i=1$ vanish by (A.14), so that we may integrate over $z_{1}$,

$$
J_{1,1}^{(1)}=\frac{2}{(\operatorname{det} Y)^{2}} \int_{\Sigma^{4}} \Delta(2,3) \Delta(4,5) \overline{\Delta(2,3) \Delta(4,5)} \sum_{2 \leq i<j \leq 5} s_{i j} \mathcal{G}(i, j)
$$

The contributions proportional to $s_{23}$ and $s_{45}$ are equal to one another. The contributions from $s_{24}, s_{25}, s_{34}, s_{35}$ are also equal to one another, so that we get,

$$
\begin{aligned}
J_{1,1}^{(1)}= & 2 \frac{s_{23}+s_{45}}{(\operatorname{det} Y)^{2}} \int_{\Sigma^{4}} \Delta(2,3) \Delta(4,5) \overline{\Delta(2,3) \Delta(4,5)} \mathcal{G}(2,3) \\
& +2 \frac{s_{24}+s_{25}+s_{34}+s_{35}}{(\operatorname{det} Y)^{2}} \int_{\Sigma^{4}} \Delta(2,3) \Delta(4,5) \overline{\Delta(2,3) \Delta(4,5)} \mathcal{G}(2,4)
\end{aligned}
$$

The integral over the point 3 in the second line is proportional to $\kappa(2)$, whose integral against $\mathcal{G}(2,4)$ vanishes, so that the second line vanishes. Integrating over the points 4,5 in the first line and using the formula for $\varphi$, we find,

$$
J_{1,1}^{(1)}=-64\left(s_{23}+s_{45}\right) \varphi
$$

The first order contributions $J_{1,2}^{(1)}$ are given by,

$$
J_{1,2}^{(1)}=\frac{1}{(\operatorname{det} Y)^{2}} \int_{\Sigma^{5}} \nu(1,2) \Delta(2,3) \overline{\Delta(3,4)} \Delta(4,5) \overline{\Delta(5,1)} \sum_{i<j} s_{i j} \mathcal{G}(i, j)
$$

For each $i<j$, we integrate over the three points that are different from $i, j$ using only the formulas of (A.12). The remaining integrals over $i, j$ are then evaluated using one of the representations of $\varphi$ in (3.13). Noting that the contributions of $s_{1 j}$ and $s_{2 j}$ for $j=3,4,5$ are equal to one another; that the contributions of $s_{24}$ and $s_{35}$ are equal to one another; and that the contributions of $s_{23}, s_{25}, s_{34}, s_{45}$ are equal to one another, we find,

$$
J_{1,2}^{(1)}=64 s_{35} \varphi
$$

where we have used momentum conservation to obtain the final result. Finally,

$$
J_{1,3}^{(1)}=\frac{1}{(\operatorname{det} Y)^{2}} \int_{\Sigma^{5}} \nu(1,3) \Delta(2,3) \Delta(4,5) \overline{\Delta(4,5) \Delta(1,2)} \sum_{i<j} s_{i j} \mathcal{G}(i, j)
$$

When $j=5$ and $i \neq 4$, as well as when $j=4$ and $i<4$, the integrals vanish because by integrating out one of the variables different from $i$ and $j$ they result in an integration of $\mathcal{G}$ against $\kappa$ which vanishes. Thus the only remaining contributions involve $s_{12}, s_{23}, s_{31}, s_{45}$, and they are readily evaluated,

$$
J_{1,3}^{(1)}=32\left(s_{12}-s_{13}+s_{23}+s_{45}\right) \varphi=64\left(s_{45}-s_{13}\right) \varphi
$$




\section{B.3.2 Second order in $s$}

To second order, we have,

$$
J_{1,1}^{(2)}=\int_{\Sigma^{5}} \kappa(1) \frac{|\Delta(2,3) \Delta(4,5)|^{2}}{(\operatorname{det} Y)^{2}} \sum_{i<j} \sum_{k<\ell} s_{i j} s_{k \ell} \mathcal{G}(i, j) \mathcal{G}(k, \ell)
$$

Each sum has 10 terms, so the total has 100 terms. However, all terms involving $s_{1 j} s_{k \ell}$ with $j, k, \ell \neq 1$ cancel, reducing the number of terms to $16+36=52$. To organize these, we proceed by evaluating first the perfect squares,

$$
\int_{\Sigma^{5}} \kappa(1) \frac{|\Delta(2,3) \Delta(4,5)|^{2}}{(\operatorname{det} Y)^{2}} \sum_{i<j} s_{i j}^{2} \mathcal{G}(i, j)^{2}
$$

for which $s_{12}^{2}, s_{13}^{2}, s_{14}^{2}, s_{15}^{2}$ all have the same coefficient $8 \mathcal{Z}_{1}$. Moreover, $s_{24}^{2}, s_{25}^{2}, s_{34}^{2}, s_{35}^{2}$ also all have the same coefficient $8 \mathcal{Z}_{1}$, and $s_{23}^{2}, s_{45}^{2}$ also have the same coefficients $16 \mathcal{Z}_{1}+8 \mathcal{Z}_{4}$. Next, we evaluate the terms involving $s_{1 j} s_{1 \ell}$ with $\ell \neq j$ (to which we refer as "angles"). For $(j, \ell)=(2,3),(4,5)$, the coefficient is $16 \mathcal{Z}_{1}$, while for $(j, \ell)=(2,4),(2,5),(3,4),(3,5)$ the coefficient vanishes because the integral over the remaining point produces a $\kappa$ which integrates to zero against $\mathcal{G}$. Finally, for $2 \leq i<j \leq 5$ and $2 \leq k<\ell \leq 5$, the coefficients of the terms $s_{23}$ times $s_{24}, s_{25}, s_{34}, s_{35}$ vanish as do their mirror images $s_{45}$ times $s_{24}, s_{25}, s_{34}, s_{35}$. The remaining terms are readily evaluated, and we find,

$$
\begin{aligned}
J_{1,1}^{(2)}= & 8 \mathcal{Z}_{1} \sum_{i<j} s_{i j}^{2}+8\left(\mathcal{Z}_{1}+\mathcal{Z}_{4}\right)\left(s_{23}^{2}+s_{45}^{2}\right)+16 \mathcal{Z}_{3}\left(s_{24} s_{35}+s_{25} s_{34}\right)+32 \varphi^{2} s_{23} s_{45} \\
& +16 \mathcal{Z}_{2}\left(s_{12} s_{13}+s_{14} s_{15}+s_{24} s_{25}+s_{24} s_{34}+s_{34} s_{35}+s_{25} s_{35}\right)
\end{aligned}
$$

Recasting the expression in terms of the cyclic variables $s_{i, i+1}$ we obtain (3.19).

Next we evaluate,

$$
J_{1,2}^{(2)}=\frac{1}{2} \int_{\Sigma^{5}} \nu(1,2) \frac{\Delta(2,3) \overline{\Delta(3,4)} \Delta(4,5) \overline{\Delta(5,1)}}{(\operatorname{det} Y)^{2}} \sum_{i<j} \sum_{k<\ell} s_{i j} s_{k \ell} \mathcal{G}(i, j) \mathcal{G}(k, \ell)
$$

The sum over $i, j, k, \ell$ again involves 100 terms. To take advantage of symmetries, we decompose the continuous products $\Delta \bar{\Delta}$ into $\nu$ and $\kappa$ using the second equation of (A.8). This will multiply the number of terms by 4 , but we can handle them using symmetry arguments, and all integrals become mechanical. We organize the calculation as follows,

$$
J_{1,2}^{(2)}=X_{1}+X_{2}+X_{3}+X_{4}
$$

with

$$
\begin{aligned}
& X_{1}=32 \int_{\Sigma^{5}} \nu(1,2) \nu(2,4) \nu(4,1) \kappa(3) \kappa(5) \sum_{i<j} \sum_{k<\ell} s_{i j} s_{k \ell} \mathcal{G}(i, j) \mathcal{G}(k, \ell) \\
& X_{2}=-16 \int_{\Sigma^{5}} \nu(1,2) \nu(2,3) \nu(3,4) \nu(4,1) \kappa(5) \sum_{i<j} \sum_{k<\ell} s_{i j} s_{k \ell} \mathcal{G}(i, j) \mathcal{G}(k, \ell) \\
& X_{3}=-16 \int_{\Sigma^{5}} \nu(1,2) \nu(2,4) \nu(4,5) \nu(5,1) \kappa(3) \sum_{i<j} \sum_{k<\ell} s_{i j} s_{k \ell} \mathcal{G}(i, j) \mathcal{G}(k, \ell) \\
& X_{4}=8 \int_{\Sigma^{5}} \nu(1,2) \nu(2,3) \nu(3,4) \nu(4,5) \nu(5,1) \sum_{i<j} \sum_{k<\ell} s_{i j} s_{k \ell} \mathcal{G}(i, j) \mathcal{G}(k, \ell)
\end{aligned}
$$


In $X_{1}$, the points 3,5 enter either into two or zero Green functions. This significantly reduces the number of contributions, and we find,

$$
\begin{aligned}
X_{1}= & 8 \mathcal{Z}_{1}\left(s_{31}^{2}+s_{32}^{2}+s_{34}^{2}+s_{35}^{2}+s_{51}^{2}+s_{52}^{2}+s_{54}^{2}\right)-8 \mathcal{Z}_{4}\left(s_{12}^{2}+s_{24}^{2}+s_{41}^{2}\right) \\
& -16 \mathcal{Z}_{2}\left(s_{31} s_{32}+s_{31} s_{34}+s_{32} s_{34}+s_{51} s_{52}+s_{51} s_{54}\right. \\
& \left.+s_{52} s_{54}+s_{12} s_{14}+s_{21} s_{24}+s_{42} s_{41}\right)
\end{aligned}
$$

In $X_{2}$, either two or zero Green functions $\mathcal{G}$ involve the point 5 , and the integration measure is cyclic symmetric in the remaining points. For squares, all terms where point 5 occurs twice have the same coefficient, while for the terms independent on point 5 , we distinguish between contributions where $\mathcal{G}$ connects contiguous points or not. For angles, we distinguish whether the angle is anchored at the point 5 or not and whether $\mathcal{G}$ connect contiguous points or not. The disconnected contributions do not involve the point 5 . The result is as follows,

$$
\begin{aligned}
X_{2}= & 4 \mathcal{Z}_{4} \sum_{i<j} s_{i j}^{2}-4\left(\mathcal{Z}_{1}+\mathcal{Z}_{4}\right)\left(s_{51}^{2}+s_{52}^{2}+s_{53}^{2}+s_{54}^{2}\right) \\
& +8 \mathcal{Z}_{2}\left(s_{51} s_{52}+s_{51} s_{53}+s_{51} s_{54}+s_{52} s_{53}+s_{52} s_{54}+s_{53} s_{54}\right. \\
& +s_{12} s_{13}+s_{12} s_{14}+s_{13} s_{14}+s_{21} s_{23}+s_{23} s_{24}+s_{21} s_{24} \\
& \left.+s_{31} s_{34}+s_{32} s_{34}+s_{32} s_{31}+s_{41} s_{42}+s_{41} s_{43}+s_{43} s_{42}\right) \\
& -16 \varphi^{2}\left(s_{12} s_{34}+s_{23} s_{14}-s_{13} s_{24}\right)-16 \mathcal{Z}_{3} s_{13} s_{24}
\end{aligned}
$$

The calculation of $X_{3}$ is analogous, but the special point is now 3 , and we find,

$$
\begin{aligned}
X_{3}= & 4 \mathcal{Z}_{4} \sum_{i<j} s_{i j}^{2}-4\left(\mathcal{Z}_{1}+\mathcal{Z}_{4}\right)\left(s_{31}^{2}+s_{32}^{2}+s_{34}^{2}+s_{35}^{2}\right) \\
& +8 \mathcal{Z}_{2}\left(s_{31} s_{32}+s_{31} s_{34}+s_{31} s_{35}+s_{32} s_{34}+s_{32} s_{35}+s_{34} s_{35}\right. \\
& +s_{12} s_{15}+s_{14} s_{15}+s_{12} s_{14}+s_{21} s_{24}+s_{21} s_{25}+s_{24} s_{25} \\
& \left.+s_{41} s_{42}+s_{42} s_{45}+s_{41} s_{45}+s_{51} s_{54}+s_{52} s_{54}+s_{51} s_{52}\right) \\
& -16 \varphi^{2}\left(s_{12} s_{45}-s_{14} s_{25}+s_{15} s_{24}\right)-16 \mathcal{Z}_{3} s_{14} s_{25}
\end{aligned}
$$

For $X_{4}$, we exploit the cyclic symmetry of the integrand. The squares of nearest neighbors have the same coefficient, and so do the squares of next-to-nearest neighbors; there are 4 classes of angles depending on the relative position of the vertex of the angle and the two other points; and there are three classes of disconnected contributions. In total we get,

$$
\begin{aligned}
X_{4}= & -2 \mathcal{Z}_{4} \sum_{i<j} s_{i j}^{2}-4 \mathcal{Z}_{2} \sum_{i} \sum_{j<k} s_{i j} s_{i k} \\
& +8 \mathcal{Z}_{3}\left(s_{13} s_{25}+s_{24} s_{31}+s_{35} s_{42}+s_{41} s_{53}+s_{52} s_{14}\right) \\
& +8 \varphi^{2}\left(s_{12} s_{34}+s_{12} s_{35}+s_{12} s_{45}-s_{13} s_{24}-s_{13} s_{25}+s_{13} s_{45}+s_{14} s_{23}-s_{14} s_{25}\right. \\
& \left.-s_{14} s_{35}+s_{15} s_{23}+s_{15} s_{24}+s_{15} s_{34}+s_{23} s_{45}-s_{24} s_{35}+s_{25} s_{34}\right)
\end{aligned}
$$


Putting all together, we have,

$$
\begin{aligned}
J_{1,2}^{(2)}= & \left(4 \mathcal{Z}_{1}+2 \mathcal{Z}_{4}\right)\left(s_{51}^{2}+s_{52}^{2}+s_{54}^{2}+s_{31}^{2}+s_{32}^{2}+s_{34}^{2}\right)-2 \mathcal{Z}_{4}\left(s_{12}^{2}+s_{14}^{2}+s_{24}^{2}+s_{35}^{2}\right) \\
& +4 \mathcal{Z}_{2}\left(s_{12} s_{13}-s_{12} s_{14}+s_{12} s_{15}+s_{13} s_{14}-s_{13} s_{15}+s_{14} s_{15}\right. \\
& +s_{23} s_{24}-s_{23} s_{25}+s_{23} s_{21}+s_{24} s_{25}-s_{24} s_{21}+s_{25} s_{21} \\
& +s_{34} s_{35}-s_{34} s_{31}-s_{34} s_{32}+s_{35} s_{31}+s_{35} s_{32}-s_{31} s_{32} \\
& +s_{45} s_{41}+s_{45} s_{42}-s_{45} s_{43}-s_{41} s_{42}+s_{41} s_{43}+s_{42} s_{43} \\
& -s_{51} s_{52}+s_{51} s_{53}-s_{51} s_{54}+s_{52} s_{53}-s_{52} s_{54}+s_{53} s_{54} \\
& -8 \varphi^{2}\left(s_{12} s_{34}+s_{23} s_{14}-s_{13} s_{24}+s_{12} s_{45}-s_{14} s_{25}+s_{15} s_{24}-s_{12} s_{35}+s_{13} s_{25}\right. \\
& \left.-s_{13} s_{45}+s_{14} s_{35}-s_{15} s_{23}-s_{15} s_{34}-s_{23} s_{45}+s_{24} s_{35}-s_{25} s_{34}\right) \\
& -8 \mathcal{Z}_{3}\left(s_{24} s_{31}+s_{52} s_{14}-s_{35} s_{42}-s_{41} s_{53}-s_{13} s_{25}\right)
\end{aligned}
$$

Recasting the expression in terms of the cyclic variables $s_{i, i+1}$ we obtain (3.19).

Next we evaluate,

$$
J_{1,3}^{(2)}=\frac{1}{2} \int_{\Sigma^{5}} \nu(1,3) \frac{\Delta(3,2) \overline{\Delta(2,1)}|\Delta(4,5)|^{2}}{(\operatorname{det} Y)^{2}} \sum_{i<j} \sum_{k<\ell} s_{i j} s_{k \ell} \mathcal{G}(i, j) \mathcal{G}(k, \ell)
$$

We proceed in analogy with $J_{1,3}^{(2)}$ and decompose as follows,

$$
J_{1,3}^{(2)}=Y_{1}+Y_{2}+Y_{3}+Y_{4}
$$

with

$$
\begin{aligned}
& Y_{1}=-64 \int_{\Sigma^{5}} \nu(1,3) \nu(3,1) \kappa(2) \kappa(4) \kappa(5) \sum_{i<j} \sum_{k<\ell} s_{i j} s_{k \ell} \mathcal{G}(i, j) \mathcal{G}(k, \ell) \\
& Y_{2}=32 \int_{\Sigma^{5}} \nu(1,3) \nu(3,2) \nu(2,1) \kappa(4) \kappa(5) \sum_{i<j} \sum_{k<\ell} s_{i j} s_{k \ell} \mathcal{G}(i, j) \mathcal{G}(k, \ell) \\
& Y_{3}=16 \int_{\Sigma^{5}} \nu(1,3) \nu(3,1) \nu(4,5) \nu(5,4) \kappa(2) \sum_{i<j} \sum_{k<\ell} s_{i j} s_{k \ell} \mathcal{G}(i, j) \mathcal{G}(k, \ell) \\
& Y_{4}=-8 \int_{\Sigma^{5}} \nu(1,3) \nu(3,2) \nu(2,1) \nu(4,5) \nu(5,4) \sum_{i<j} \sum_{k<\ell} s_{i j} s_{k \ell} \mathcal{G}(i, j) \mathcal{G}(k, \ell)
\end{aligned}
$$

The combinatorics is similar to $J_{1,3}^{(2)}$ and the integrals are readily recognized,

$$
\begin{aligned}
Y_{1}= & 16\left(\mathcal{Z}_{1}+\mathcal{Z}_{4}\right) s_{13}^{2}-16 \mathcal{Z}_{1} \sum_{i<j} s_{i j}^{2}+32 \mathcal{Z}_{2}\left(s_{21} s_{23}+s_{41} s_{43}+s_{51} s_{53}\right) \\
Y_{2}= & -8\left(\mathcal{Z}_{1}+\mathcal{Z}_{4}\right)\left(s_{12}^{2}+s_{23}^{2}+s_{31}^{2}\right)+8 \mathcal{Z}_{1} \sum_{i<j} s_{i j}^{2} \\
& -16 \mathcal{Z}_{2}\left(s_{12} s_{13}+s_{21} s_{23}+s_{31} s_{32}+s_{41} s_{42}+s_{41} s_{43}+s_{42} s_{43}+s_{51} s_{52}+s_{51} s_{53}+s_{52} s_{53}\right)
\end{aligned}
$$




$$
\begin{aligned}
Y_{3}= & -8\left(\mathcal{Z}_{1}+\mathcal{Z}_{4}\right)\left(s_{13}^{2}+s_{45}^{2}\right)+8 \mathcal{Z}_{1} \sum_{i<j} s_{i j}^{2}+32 \varphi^{2} s_{13} s_{45}+16 \mathcal{Z}_{3}\left(s_{14} s_{35}+s_{15} s_{34}\right) \\
& -16 \mathcal{Z}_{2}\left(s_{12} s_{23}+s_{14} s_{15}+s_{24} s_{25}+s_{34} s_{35}+s_{14} s_{34}+s_{15} s_{35}\right) \\
Y_{4}= & 4 \mathcal{Z}_{4}\left(s_{12}^{2}+s_{13}^{2}+s_{23}^{2}+s_{45}^{2}\right)-4 \mathcal{Z}_{1}\left(s_{14}^{2}+s_{15}^{2}+s_{24}^{2}+s_{25}^{2}+s_{34}^{2}+s_{35}^{2}\right) \\
& -16 \varphi^{2}\left(s_{12}+s_{23}+s_{31}\right) s_{45}-8 \mathcal{Z}_{3}\left(s_{14} s_{25}+s_{14} s_{35}+s_{24} s_{15}+s_{24} s_{35}+s_{34} s_{15}+s_{34} s_{25}\right) \\
& +8 \mathcal{Z}_{2}\left(s_{12} s_{13}+s_{13} s_{23}+s_{12} s_{23}+s_{41} s_{42}+s_{42} s_{43}+s_{43} s_{41}\right. \\
& \left.+s_{51} s_{52}+s_{52} s_{53}+s_{53} s_{51}+s_{14} s_{15}+s_{24} s_{25}+s_{34} s_{35}\right)
\end{aligned}
$$

Assembling all contributions, we find the following equivalent of the $s_{i j}^{2}$ order in (3.19),

$$
\begin{aligned}
J_{1,3}^{(2)}= & -4 \mathcal{Z}_{1}\left(2 s_{12}^{2}+2 s_{23}^{2}+2 s_{45}^{2}+s_{14}^{2}+s_{15}^{2}+s_{24}^{2}+s_{25}^{2}+s_{34}^{2}+s_{35}^{2}\right) \\
& -4 \mathcal{Z}_{4}\left(s_{12}^{2}+s_{23}^{2}-s_{31}^{2}+s_{45}^{2}\right)-16 \varphi^{2}\left(s_{12}+s_{23}-s_{13}\right) s_{45} \\
& -8 \mathcal{Z}_{3}\left(s_{14} s_{25}-s_{14} s_{35}+s_{24} s_{15}+s_{24} s_{35}-s_{34} s_{15}+s_{34} s_{25}\right) \\
& +8 \mathcal{Z}_{2}\left(-s_{12} s_{13}-s_{13} s_{23}+s_{12} s_{23}-s_{41} s_{42}-s_{42} s_{43}+s_{43} s_{41}\right. \\
& \left.-s_{51} s_{52}-s_{52} s_{53}+s_{53} s_{51}-s_{14} s_{15}-s_{24} s_{25}-s_{34} s_{35}\right)
\end{aligned}
$$

One readily verifies that the result is symmetric in 1,3 as well as in 4,5 . Recasting the expression in terms of the cyclic variables $s_{i, i+1}$ we obtain (3.19).

\section{B.4 The $F$-integrals}

Finally, we turn to the $F$-integrals defined in (3.4). As stressed in section 3.2, these integrals have a simple pole at $s_{12}=0$, which can be exposed by means of the identity (3.8). Applying the same method as for (3.10), we get,

$$
\begin{aligned}
F_{2}= & -\frac{1}{i \pi} \sum_{k=3}^{5} \frac{s_{1 k}}{s_{12}} \int_{\Sigma^{5}} \frac{\mathrm{KN}_{(5)}}{(\operatorname{det} Y)^{2}} \partial_{1} \mathcal{G}(1,2) \bar{\partial}_{1} \mathcal{G}(1, k) \Delta(2,3) \Delta(4,5) \overline{\Delta(2,4) \Delta(3,5)} \\
& +\frac{2}{s_{12}} \int_{\Sigma^{5}} \frac{\mathrm{KN}_{(5)}}{(\operatorname{det} Y)^{2}} \kappa(1) \Delta(2,3) \Delta(4,5) \overline{\Delta(2,4) \Delta(3,5)} \\
F_{3}= & -\frac{1}{i \pi} \sum_{k=3}^{5} \frac{s_{1 k}}{s_{12}} \int_{\Sigma^{5}} \frac{\mathrm{KN}_{(5)}}{(\operatorname{det} Y)^{2}} \partial_{1} \mathcal{G}(1,2) \bar{\partial}_{2} \mathcal{G}(2, k) \Delta(2,3) \overline{\Delta(1,3)}|\Delta(4,5)|^{2} \\
& -\frac{2}{s_{12}} \int_{\Sigma^{5}} \frac{\mathrm{KN}_{(5)}}{(\operatorname{det} Y)^{2}} \nu(1,2) \Delta(2,3) \overline{\Delta(1,3)|\Delta(4,5)|^{2}} \\
F_{4}= & -\frac{1}{i \pi} \sum_{k=3}^{5} \frac{s_{1 k}}{s_{12}} \int_{\Sigma^{5}} \frac{\mathrm{KN}_{(5)}}{(\operatorname{det} Y)^{2}} \partial_{1} \mathcal{G}(1,2) \bar{\partial}_{2} \mathcal{G}(2, k) \Delta(2,3) \Delta(4,5) \overline{\Delta(1,4) \Delta(3,5)} \\
& -\frac{2}{s_{12}} \int_{\Sigma^{5}} \frac{\mathrm{KN}_{(5)}}{(\operatorname{det} Y)^{2}} \nu(1,2) \Delta(2,3) \Delta(4,5) \overline{\Delta(1,4) \Delta(3,5)}
\end{aligned}
$$

For each $F_{a}$, the first term on the right side may be expressed as a linear combination of the functions $G_{1}$ and $G_{2}$, whose $\alpha^{\prime}$ expansion was computed in section B.2 and given in (3.21). The second term on the right may be expressed as a linear combination of the 
functions $J_{r, s}$, whose $\alpha^{\prime}$ expansion was computed in section B.3 and given in (3.19). As a result of the integration-by-parts relations, we obtain,

$$
\begin{aligned}
& s_{12} F_{1}=J_{1,1}-s_{13}\left(G_{1}+\left.G_{1}\right|_{4 \leftrightarrow 5}-G_{2}-\left.G_{2}\right|_{4 \leftrightarrow 5}\right)-s_{14}\left(\left.G_{1}\right|_{3 \leftrightarrow 4}\right)-s_{15}\left(\left.G_{1}\right|_{5,3,4} ^{3,4,5} \begin{array}{c}
\downarrow \\
5
\end{array}\right) \\
& s_{12} F_{2}=J_{1,1}-J_{1,2}-J_{1,5}-s_{13}\left(G_{1}-\left.G_{2}\right|_{4 \leftrightarrow 5}\right) \\
& -s_{14}\left(\left.G_{1}\right|_{3 \leftrightarrow 4}-\left.G_{2}\right|_{3 \leftrightarrow 4}\right)-s_{15}\left(G_{2} \mid \begin{array}{c}
3,4,5 \\
\downarrow \\
5,3,4
\end{array}\right)
\end{aligned}
$$

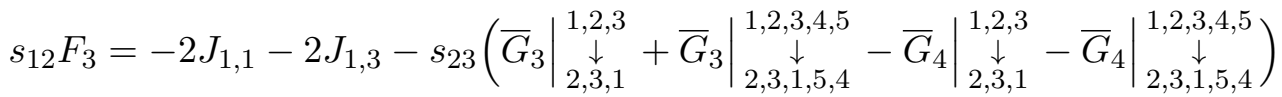

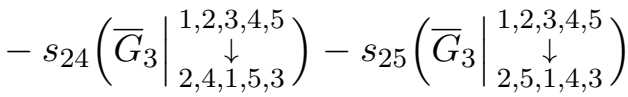

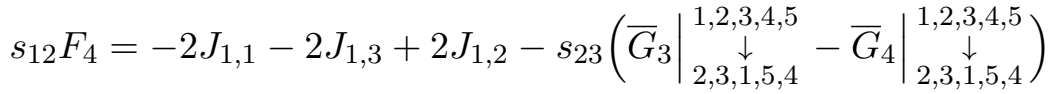

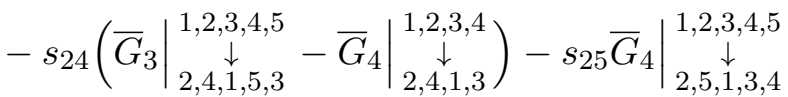

which yields the expansions of $F_{j}$ in (3.20). The transpositions and permutations annotated on the right of the $\mid \ldots$ act on the external momenta in the $\alpha^{\prime}$ expansion of the respective integrals. We note that $J_{1,5}$ may be evaluated in terms of $J_{1,2}$ by using the relation,

$$
J_{1,5}=\bar{J}_{1,2} \mid \begin{gathered}
1,2,3,4,5 \\
\downarrow \\
5,1,2,3,4
\end{gathered}
$$

The complex conjugations on $G$ in the expressions for $F_{3}, F_{4}$ and $J_{1,2}$ do not complex conjugate the kinematic variables $s_{i j}$.

\section{Degenerations of genus-two modular graph functions}

In this appendix, we shall obtain the non-separating degeneration, the separating degeneration, and the tropical limit of the modular graph functions which are needed to order $D^{6} \mathcal{R}^{5}$ in the analysis of the 5-point amplitude. Standard mathematical references on degenerations of Riemann surfaces are [90, 91]. Here we shall briefly review the methods developed in $[31,32]$ to obtain the non-separating and tropical degenerations of higher genus modular graph functions, restricted here to the application to genus two.

\section{C.1 The non-separating degeneration}

To describe the non-separating degeneration of a genus-two surface $\Sigma$ to a genus-one surface, it is useful to parametrize the period matrix $\Omega$ of the Riemann surface $\Sigma$ as follows,

$$
\Omega=\left(\begin{array}{cc}
\tau & v \\
v & \sigma_{1}+i\left(t+\tau_{2} u_{2}^{2}\right)
\end{array}\right) \quad Y=\operatorname{Im} \Omega=\left(\begin{array}{cc}
\tau_{2} & \tau_{2} u_{2} \\
\tau_{2} u_{2} & t+\tau_{2} u_{2}^{2}
\end{array}\right)
$$

where $\tau=\tau_{1}+i \tau_{2}$ and $v=u_{1}+\tau u_{2}$ with $\tau_{1}, \tau_{2}, u_{1}, u_{2}, \sigma_{1}, t \in \mathbb{R}$ and $\tau_{2}, t>0$. The non-separating degeneration corresponds to the limit $t \rightarrow \infty$ keeping $\tau, v$ and $\sigma_{1}$ fixed.

Actually, to obtain the desired expansion of modular graph functions, we shall be interested not just in the non-separating degeneration limit (which is a genus-one surface with 


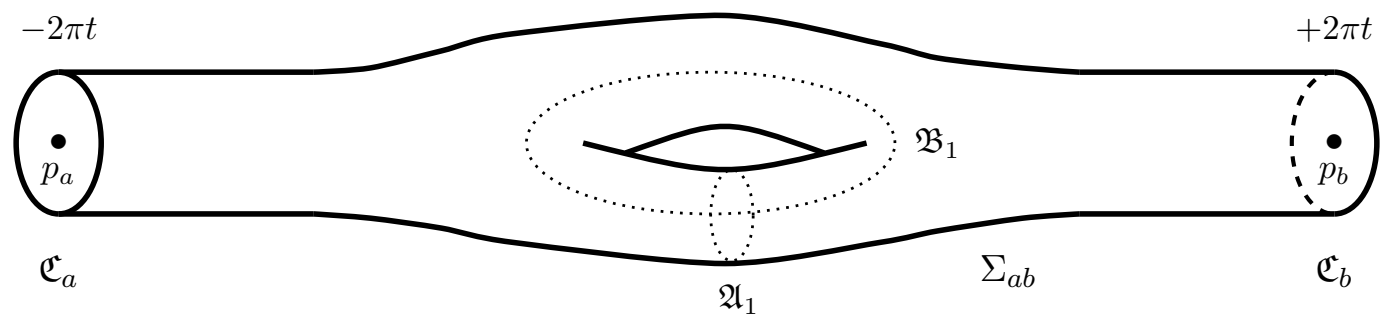

Figure 3. The surface $\Sigma_{a b}$ is obtained from $\Sigma$ in the vicinity of the non-separating degeneration limit by cutting $\Sigma$ along a cycle homologous to $\mathfrak{A}_{2}$ and adjusting the position of the cycle so that $\mathfrak{C}_{a}$ and $\mathfrak{C}_{b}$ are level sets for $f= \pm 2 \pi t$.

two punctures $\left.p_{a}, p_{b}\right)$, but in a small but finite neighborhood of this limit. To parametrize this neighborhood, we reconstruct the genus-two surface $\Sigma$ from a genus-one surface $\Sigma_{a b}$ with two disconnected boundary discs $\mathfrak{C}_{a}, \mathfrak{C}_{b}$ as shown in figure 3 . The surface $\Sigma_{a b}$ may be obtained from an underlying compact genus-one surface $\Sigma_{1}$ with modulus $\tau$ and two marked points $p_{a}, p_{b}$ obeying $v=p_{b}-p_{a}$ from which the discs $\mathfrak{C}_{a}, \mathfrak{C}_{b}$ centered at $p_{a}, p_{b}$ of radius $R$ have been removed. Note that the points $p_{a}, p_{b}$ belong to $\Sigma_{1}$ but not to $\Sigma_{a b}$. The genus-two surface $\Sigma$ is obtained by gluing annular neighborhoods of size $t$ of the boundary curves $\mathfrak{C}_{a}, \mathfrak{C}_{b}$ together. The details of the construction may be found in [31, 32].

To describe this construction concretely, it is useful to introduce the linear combination $\omega_{t}=\omega_{2}-u_{2} \omega_{1}$ of holomorphic $(1,0)$ forms on $\Sigma$, such that the canonical forms defined in (3.11), (A.6) decompose as follows,

$$
\begin{aligned}
\kappa(x) & =\frac{i}{4 \tau_{2}} \omega_{1}(x) \bar{\omega}_{1}(x)+\frac{i}{4 t} \omega_{t}(x) \bar{\omega}_{t}(x) \\
\nu(x, y) & =\frac{i}{2 \tau_{2}} \omega_{1}(x) \bar{\omega}_{1}(y)+\frac{i}{2 t} \omega_{t}(x) \bar{\omega}_{t}(y) \\
\Delta(x, y) & =\omega_{1}(x) \omega_{t}(y)-\omega_{t}(x) \omega_{1}(y)
\end{aligned}
$$

In the limit $t \rightarrow \infty$ for a fixed point $x \in \Sigma_{a b}$, the holomorphic $(1,0)$ forms behave as follows,

$$
\omega_{1}=1+\mathcal{O}\left(e^{-2 \pi t}\right) \quad \omega_{t}=\frac{i}{2 \pi} \partial_{x} f(x)+\mathcal{O}\left(e^{-2 \pi t}\right)
$$

Here, the real-valued function $f(x)$ plays the role of a Morse function on $\Sigma_{a b}$ and may be given explicitly in terms of the genus-one Arakelov Green function $g(x, y)=g(x-y \mid \tau)$ on $\Sigma_{1}$ by the following exact formula,

$$
f(x)=g\left(x, p_{b}\right)-g\left(x, p_{a}\right) \quad v=p_{b}-p_{a}
$$

The discs $\mathfrak{C}_{a}$ and $\mathfrak{C}_{b}$ may be specified concretely by the conditions $f\left(\mathfrak{C}_{a}\right)=-2 \pi t$ and $f\left(\mathfrak{C}_{b}\right)=+2 \pi t$, as shown in figure 3 . For sufficiently large $t$, the discs will be disjoint.

The Arakelov Green function $\mathcal{G}(x, y)$ has an exact asymptotic expansion as $t \rightarrow \infty$, for fixed $x, y \in \Sigma_{a b}$, given by [31],

$$
\mathcal{G}(x, y)=\tilde{G}(x, y)+\tilde{\gamma}(x)+\tilde{\gamma}(y)+\tilde{\gamma}_{0}+\mathcal{O}\left(e^{-2 \pi t}\right)
$$


where the terms in the sum are given by,

$$
\begin{aligned}
\tilde{G}(x, y) & =g(x, y)-\frac{f(x) f(y)}{4 \pi t} \\
\tilde{\gamma}(x) & =-\frac{1}{4} g\left(x, p_{a}\right)-\frac{1}{4} g\left(x, p_{b}\right)+\frac{f(x)^{2}}{16 \pi t} \\
\tilde{\gamma}_{0} & =\frac{\pi t}{12}+\frac{1}{4} g(v)-\frac{F_{2}(v)}{8 \pi t}
\end{aligned}
$$

and $F_{k}$ is the genus-one elliptic modular graph function defined in (3.17). For later use, it will be useful to further introduce combinations familiar from [32],

$$
\begin{aligned}
D_{k}(\tau) & =\int_{\Sigma_{1}} \kappa_{1}(z) g(z)^{k} \\
D_{k}^{(\ell)}(v \mid \tau) & =\int_{\Sigma_{1}} \kappa_{1}(z) g(z, v)^{\ell} g(z)^{k-\ell} \\
g_{k+1}(v \mid \tau) & =\int_{\Sigma_{1}} \kappa_{1}(z) g(z, v) g_{k}(z)
\end{aligned}
$$

such that $g_{1}(v \mid \tau)=g(v \mid \tau), D_{k}^{(\ell)}(0 \mid \tau)=D_{k}(\tau)$, and $g_{k}(0 \mid \tau)=E_{k}(\tau)$, where $E_{k}$ is the non-holomorphic Eisenstein series (4.57). Henceforth we shall suppress the dependence on $v$ and $\tau$.

\section{C.1.1 Useful integrals}

To expand the genus-two integrals near the non-separating degeneration, the following simple integrals over $\Sigma_{a b}$ will be needed,

$$
\begin{aligned}
\int_{\Sigma_{a b}} \omega_{t} \bar{\omega}_{1} f^{n} & =\int_{\Sigma_{a b}} \omega_{t} \overline{\omega_{t}} f^{2 n+1}=0 \\
\int_{\Sigma_{a b}} \omega_{t} \overline{\omega_{t}} f^{2 n} & =-\frac{2 i}{2 n+1}(2 \pi)^{2 n} t^{2 n+1} \\
\int_{\Sigma_{a b}} \omega_{t}(y) \bar{\omega}_{1}(y) g_{n}(y-x) & =\frac{\tau_{2}}{\pi} \partial_{x} f_{n+1}
\end{aligned}
$$

where we define $f_{n}(x)=g_{n}\left(x-p_{b}\right)-g_{n}\left(x-p_{a}\right)$, such that $f_{1}=f$. For any function $\psi(x)$ which is smooth on $\Sigma_{a b}$ and whose Laplacian $\partial_{x} \partial_{\bar{x}} \psi(x)$ is smooth on $\Sigma_{a b}$, but which does not need to extend to a smooth function at the punctures $x=p_{a}, p_{b}$, we have, ${ }^{24}$

$$
\begin{aligned}
\int_{\Sigma_{a b}} \omega_{t} \overline{\omega_{t}} f^{n} \psi= & -\frac{i(2 \pi t)^{n+1}}{4 \pi^{2}(n+1)} \int_{0}^{2 \pi} d \theta\left(\psi\left(p_{b}^{\theta}\right)+(-)^{n} \psi\left(p_{a}^{\theta}\right)\right) \\
& -\frac{i(2 \pi t)^{n+2}}{8 \pi^{2}(n+1)(n+2)} \int_{0}^{2 \pi} d \theta R \frac{\partial}{\partial R}\left(\psi\left(p_{b}^{\theta}\right)+(-)^{n} \psi\left(p_{a}^{\theta}\right)\right) \\
& -\frac{i \tau_{2}}{2 \pi^{2}(n+1)(n+2)} \int_{\Sigma_{a b}} \kappa_{1}(z) f(z)^{n+2} \partial_{z} \partial_{\bar{z}} \psi(z)
\end{aligned}
$$

\footnotetext{
${ }^{24}$ The middle term was omitted in equation (A.21) of [32], but its effect was correctly included in the subsequent equations in section A.5 of that reference.
} 
where $p_{a, b}^{\theta}=p_{a, b}+R e^{i \theta}$ and $\theta$ is the coordinate on the boundary circles $\mathfrak{C}_{a}, \mathfrak{C}_{b}$. The relation between $R$ and $t$ is given by the behavior of the scalar Green function for nearly coinciding points,

$$
g(z-p \mid \tau)=2 \pi t-\ln \frac{|z-p|^{2}}{R^{2}}+g(v \mid \tau)+\mathcal{O}(z-p)
$$

for $p=p_{a}, p_{b}$. Using these fundamental formulas, we derive integrals required to evaluate the non-separating asymptotics of $\varphi, \mathcal{Z}_{1}, \mathcal{Z}_{2}, \mathcal{Z}_{3}, \mathcal{Z}_{4}, \mathcal{Z}_{5}$ and, abbreviating $g=g(v \mid \tau)$, we get,

$$
\begin{aligned}
2 \pi i \int_{\Sigma_{a b}} \omega_{t}(z) \bar{\omega}_{t}(z) g\left(z, p_{a, b}\right) & =2 \pi^{2} t^{2}+4 \pi t g+F_{2} \\
-\frac{i}{4 t} \int_{\Sigma_{a b}} \omega_{t}(z) \bar{\omega}_{t}(z) g\left(z, p_{a, b}\right)^{2} & =-\frac{\pi^{2} t^{2}}{3}-\frac{\pi t}{2} g-\frac{1}{2} g^{2}-\frac{D_{3}-D_{3}^{(1)}}{8 \pi t}-\frac{\Delta_{v} F_{4}}{16 \pi^{2} t} \\
-\frac{i}{4 t} \int_{\Sigma_{a b}} \omega_{t}(z) \bar{\omega}_{t}(z) g\left(z, p_{a}\right) g\left(z, p_{b}\right) & =-\frac{\pi t}{2} g-\frac{1}{2} g^{2}-\frac{D_{3}-D_{3}^{(1)}}{8 \pi t}-\frac{\Delta_{v} F_{4}}{16 \pi^{2} t}
\end{aligned}
$$

The Laplacian on $v$ is defined by $\Delta_{v}=4 \tau_{2} \partial_{v} \partial_{\bar{v}}$.

\section{C.1.2 Non-separating degeneration of $\varphi$ and $\mathcal{Z}_{1,2,3}$}

Using the formulae above, and some further identities derived from them, the asymptotic expansion of the Kawazumi-Zhang invariant may be derived and gives,

$$
\varphi=\frac{1}{6} \pi t+\frac{1}{2} g+\frac{5 F_{2}}{4 \pi t}+\mathcal{O}\left(e^{-2 \pi t}\right)
$$

while the asymptotics of the integrals $\mathcal{Z}_{1,2,3}$ in (3.14) was obtained in equation (3.21) of [32],

$$
\begin{aligned}
\mathcal{Z}_{1}= & \frac{13 \pi^{2} t^{2}}{90}+\frac{\pi t}{3} g+\frac{E_{2}+g^{2}-F_{2}}{2}+\frac{1}{\pi t}\left(-D_{3}-D_{3}^{(1)}-\frac{1}{2} g F_{2}+2 g_{3}\right. \\
& \left.+4 \zeta_{3}+\frac{\Delta_{v} F_{4}}{4 \pi}\right)+\frac{1}{8 \pi^{2} t^{2}}\left(3 F_{2}^{2}+12 F_{4}+\mathcal{K}^{c}\right)+\mathcal{O}\left(e^{-2 \pi t}\right) \\
\mathcal{Z}_{2}= & -\frac{7 \pi^{2} t^{2}}{90}-\frac{\pi t}{3} g-\frac{2 E_{2}+g^{2}-F_{2}}{2}+\frac{1}{\pi t}\left(-2 D_{3}+\frac{1}{2} g F_{2}+2 g_{3}+2 \zeta_{3}\right. \\
& \left.-\frac{\Delta_{v}\left(F_{2}^{2}+2 F_{4}\right)}{16 \pi}\right)-\frac{\left(\Delta_{\tau}+5\right) F_{4}}{4 \pi^{2} t^{2}}+\mathcal{O}\left(e^{-2 \pi t}\right) \\
\mathcal{Z}_{3}= & \frac{(\pi t)^{2}}{18}+\frac{\pi t}{3} g+\frac{1}{6}\left(F_{2}+3 g^{2}\right)+\frac{1}{\pi t}\left(-\frac{1}{2} g F_{2}+\frac{\Delta_{v} F_{2}^{2}}{8 \pi}\right)+\frac{\left(\Delta_{\tau}+5\right) F_{2}^{2}}{8 \pi^{2} t^{2}}+\mathcal{O}\left(e^{-2 \pi t}\right)
\end{aligned}
$$

where the Laplacian on $\tau$ is defined by $\Delta_{\tau}=4 \tau_{2}^{2} \partial_{\tau} \partial_{\bar{\tau}}$ while $\mathcal{K}^{c}$ in $\mathcal{Z}_{1}$ is the (complicated) regularized integral defined in equation (3.40) of [32], which depends on $\tau$ but not on $v$.

\section{C.1.3 Non-separating degeneration of $\mathcal{Z}_{4}$}

Using (A.12), the integral $\mathcal{Z}_{4}$ defined in (3.14) can be recast as follows,

$$
\mathcal{Z}_{4}+2 \mathcal{Z}_{1}=-\int_{\Sigma^{2}} \frac{|\Delta(x, y)|^{2}}{\operatorname{det} Y} \mathcal{G}(x, y)^{2}
$$


The computation of the asymptotics of the integral is similar to the one for $\mathcal{Z}_{1}$ in [32]. Using the last identity in (C.2) we decompose it into $\mathcal{Z}_{4}+2 \mathcal{Z}_{1}=\mathcal{Z}_{-}-\mathcal{Z}_{+}$, where

$$
\begin{aligned}
& \mathcal{Z}_{+}=\frac{2}{\tau_{2} t} \int_{\Sigma_{a b}} \mathcal{G}(x, y)^{2} \omega_{1}(x) \bar{\omega}_{1}(x) \omega_{t}(y) \bar{\omega}_{t}(y) \\
& \mathcal{Z}_{-}=\frac{2}{\tau_{2} t} \int_{\Sigma_{a b}} \mathcal{G}(x, y)^{2} \omega_{1}(x) \bar{\omega}_{t}(x) \omega_{t}(y) \bar{\omega}_{1}(y)
\end{aligned}
$$

Substituting (C.5) into these equations, we get $\mathcal{Z}_{ \pm}=\mathcal{Z}_{ \pm}^{(a)}+\mathcal{Z}_{ \pm}^{(b)}+\mathcal{Z}_{ \pm}^{(c)}+\mathcal{O}\left(e^{-2 \pi t}\right)$, where

$$
\begin{aligned}
& \mathcal{Z}_{+}^{(a)}=\frac{2}{\tau_{2} t} \int_{\Sigma_{a b}} \tilde{G}(x, y)^{2} \omega_{1}(x) \bar{\omega}_{1}(x) \omega_{t}(y) \bar{\omega}_{t}(y) \\
& \mathcal{Z}_{+}^{(b)}=\frac{4}{\tau_{2} t} \int_{\Sigma_{a b}} \tilde{G}(x, y)\left(\tilde{\gamma}(x)+\tilde{\gamma}(y)+\tilde{\gamma}_{0}\right) \omega_{1}(x) \bar{\omega}_{1}(x) \omega_{t}(y) \bar{\omega}_{t}(y) \\
& \mathcal{Z}_{+}^{(c)}=\frac{2}{\tau_{2} t} \int_{\Sigma_{a b}}\left(\tilde{\gamma}(x)+\tilde{\gamma}(y)+\tilde{\gamma}_{0}\right)^{2} \omega_{1}(x) \bar{\omega}_{1}(x) \omega_{t}(y) \bar{\omega}_{t}(y)
\end{aligned}
$$

and

$$
\begin{aligned}
& \mathcal{Z}_{-}^{(a)}=\frac{2}{\tau_{2} t} \int_{\Sigma_{a b}} \tilde{G}(x, y)^{2} \omega_{1}(x) \bar{\omega}_{t}(x) \omega_{t}(y) \bar{\omega}_{1}(y) \\
& \mathcal{Z}_{-}^{(b)}=\frac{4}{\tau_{2} t} \int_{\Sigma_{a b}} \tilde{G}(x, y)\left(\tilde{\gamma}(x)+\tilde{\gamma}(y)+\tilde{\gamma}_{0}\right) \omega_{1}(x) \bar{\omega}_{t}(x) \omega_{t}(y) \bar{\omega}_{1}(y) \\
& \mathcal{Z}_{-}^{(c)}=\frac{2}{\tau_{2} t} \int_{\Sigma_{a b}}\left(\tilde{\gamma}(x)+\tilde{\gamma}(y)+\tilde{\gamma}_{0}\right)^{2} \omega_{1}(x) \bar{\omega}_{t}(x) \omega_{t}(y) \bar{\omega}_{1}(y)
\end{aligned}
$$

These integrals can be computed using the same techniques as in [32]:

- For $\mathcal{Z}_{+}^{(a)}$, using (C.9) we get

$$
\begin{aligned}
\mathcal{Z}_{+}^{(a)} & =-\frac{4 i}{t} \int_{\Sigma_{a b}} \kappa_{1}(x)\left(g(x, y)^{2}-g(x, y) \frac{f(x) f(y)}{2 \pi t}+\frac{f(x)^{2} f(y)^{2}}{16 \pi^{2} t^{2}}\right) \omega_{t}(y) \bar{\omega}_{t}(y) \\
& =-8 E_{2}+\frac{8}{3} F_{2}-\frac{4 F_{4}}{\pi^{2} t^{2}}
\end{aligned}
$$

- For $\mathcal{Z}_{+}^{(b)}$, using (C.10) and (C.11) we get

$$
\begin{aligned}
\mathcal{Z}_{+}^{(b)} & =\frac{2 i}{t} \int_{\Sigma_{a b}} \kappa_{1}(x) g(x, y)\left(g\left(x, p_{a}\right)+g\left(x, p_{b}\right)-\frac{f(x)^{2}}{4 \pi t}\right) \omega_{t}(y) \bar{\omega}_{t}(y) \\
& =4 E_{2}+4 g_{2}-\frac{2}{\pi t}\left(D_{3}-D_{3}^{(1)}\right)+\frac{6 F_{4}-F_{2}^{2}}{2 \pi^{2} t^{2}}
\end{aligned}
$$

- For $\mathcal{Z}_{+}^{(c)}$, expanding in powers of $\tilde{\gamma}(y)$ we get

$$
\begin{aligned}
\mathcal{Z}_{+}^{(c)}= & -8 \int_{\Sigma_{1}} \kappa_{1}(x)\left(\tilde{\gamma}(x)+\tilde{\gamma}_{0}\right)^{2}-\frac{8 i}{t}\left(\tilde{\gamma}_{0}+\frac{F_{2}}{8 \pi t}\right) \int_{\Sigma_{a b}} \tilde{\gamma}(y) \omega_{t}(y) \bar{\omega}_{t}(y) \\
& -\frac{4 i}{t} \int_{\Sigma_{a b}} \tilde{\gamma}(y)^{2} \omega_{t}(y) \bar{\omega}_{t}(y)
\end{aligned}
$$


Using (C.13) the three terms evaluate to the three lines below,

$$
\begin{aligned}
\mathcal{Z}_{+}^{(c)}= & -8 \tilde{\gamma}_{0}^{2}-2 \tilde{\gamma}_{0} \frac{F_{2}}{\pi t}-E_{2}-g_{2}+\frac{1}{2 \pi t}\left(D_{3}-D_{3}^{(1)}\right)-\frac{3 F_{4}}{4 \pi^{2} t^{2}} \\
& +\left(\tilde{\gamma}_{0}+\frac{F_{2}}{8 \pi t}\right)\left(\frac{2 F_{2}}{\pi t}+8 g+\frac{8 \pi t}{3}\right) \\
& -\frac{4 \pi^{2} t^{2}}{15}-\frac{4 \pi t}{3} g-2 g^{2}-\frac{\Delta_{v} F_{4}}{4 \pi^{2} t}-\frac{1}{2 \pi t}\left(D_{3}-D_{3}^{(1)}\right)+\frac{F_{4}}{4 \pi^{2} t^{2}}
\end{aligned}
$$

- For $\mathcal{Z}_{-}^{(a)}$, integrating by parts using $\tau_{2} \partial_{\bar{x}} \partial_{y} g(x, y)=\pi \delta(x, y)-\pi$ we get

$$
\begin{aligned}
\mathcal{Z}_{-}^{(a)} & =\frac{2}{\tau_{2} t} \int_{\Sigma_{a b}}\left(g(x, y)^{2}-g(x, y) \frac{f(x) f(y)}{2 \pi t}\right) \omega_{1}(x) \bar{\omega}_{t}(x) \omega_{t}(y) \bar{\omega}_{1}(y) \\
& =-\frac{2 \tau_{2}}{\pi^{2} t} \int_{\Sigma_{1}} \kappa_{1}(x) \kappa_{1}(y) g(x, y)^{2} \partial_{\bar{x}} f(x) \partial_{y} f(y)+\frac{6 F_{4}-F_{2}^{2}}{\pi^{2} t^{2}} \\
& =-\frac{4}{\pi t}\left(D_{3}-D_{3}^{(1)}\right)+\frac{6 F_{4}-F_{2}^{2}}{\pi^{2} t^{2}}
\end{aligned}
$$

- For $\mathcal{Z}_{-}^{(b)}$, integrating by parts using $\partial_{x} \partial_{\bar{x}} f_{2}=-\pi f / \tau_{2}$, we get

$$
\begin{aligned}
\mathcal{Z}_{-}^{(b)} & =\frac{2}{\tau_{2} t} \int_{\Sigma_{a b}} g(x, y)\left(2 \tilde{\gamma}(x)+\tilde{\gamma}_{0}\right) \omega_{1}(x) \bar{\omega}_{t}(x) \omega_{t}(y) \bar{\omega}_{1}(y)+\text { c.c. } \\
& =-\frac{2 \tau_{2}}{\pi^{2} t} \int_{\Sigma_{a b}} \kappa_{1}(x)\left(\partial_{x} f_{2}(x) \partial_{\bar{x}} f+\partial_{\bar{x}} f_{2}(x) \partial_{x} f(x)\right)\left(2 \tilde{\gamma}(x)+\tilde{\gamma}_{0}\right) \\
& =\frac{\mathfrak{Z}_{-}^{(b)}}{\pi t}-\frac{4 F_{4}}{\pi^{2} t^{2}}-8 \tilde{\gamma}_{0} \frac{F_{2}}{\pi t}
\end{aligned}
$$

where the term $\mathfrak{Z}_{-}^{(b)}$ originates from the contributions $g\left(x, p_{a}\right)+g\left(x, p_{b}\right)$ inside $\tilde{\gamma}(x)$. It can be evaluated again by substituting $f=g\left(x, p_{b}\right)-g\left(x, p_{a}\right)$ and integrating by parts,

$$
\begin{aligned}
\mathfrak{Z}_{-}^{(b)} & =\frac{\tau_{2}}{\pi} \int \kappa_{1}(x)\left(\partial_{x} f_{2}(x) \partial_{\bar{x}} f(x)+\partial_{\bar{x}} f_{2}(x) \partial_{x} f(x)\right)\left(g\left(x, p_{a}\right)+g\left(x, p_{b}\right)\right) \\
& =2 D_{3}-2 D_{3}^{(1)}+4 g F_{2}+4 E_{3}-4 g_{3}
\end{aligned}
$$

- For $\mathcal{Z}_{-}^{(c)}$, using (C.10) and $\tau_{2}\left|\partial f_{2}\left(p_{a}\right)\right|^{2}=\frac{1}{8 \pi} \Delta_{v}\left(F_{2}^{2}\right)-g F_{2}$ we get

$$
\begin{aligned}
\mathcal{Z}_{-}^{(c)} & =\frac{4}{\tau_{2} t} \int_{\Sigma_{a b}} \tilde{\gamma}(x) \tilde{\gamma}(y) \omega_{1}(x) \bar{\omega}_{t}(x) \omega_{t}(y) \bar{\omega}_{1}(y) \\
& =-\frac{\tau_{2}}{4 \pi^{2} t}\left|\partial_{p_{a}} f_{2}\left(p_{a}\right)+\partial_{p_{b}} f_{2}\left(p_{b}\right)\right|^{2}=-\frac{1}{8 \pi^{2} t}\left(\Delta_{v} F_{2}^{2}-8 \pi g F_{2}\right)
\end{aligned}
$$

Collecting all terms, we find

$$
\begin{aligned}
\mathcal{Z}_{+}= & -\frac{\pi^{2} t^{2}}{10}-\frac{\pi t}{3} g-2 E_{2}-\frac{F_{2}}{6}-\frac{1}{2} g^{2}-\frac{4 D_{3}-4 D_{3}^{(1)}-g F_{2}}{2 \pi t} \\
& -\frac{\Delta_{v} F_{4}}{4 \pi^{2} t}-\frac{3 F_{2}^{2}+12 F_{4}}{8 \pi^{2} t^{2}}+\mathcal{O}\left(e^{-2 \pi t}\right) \\
\mathcal{Z}_{-}= & -\frac{2}{3} F_{2}-\frac{2 D_{3}-2 D_{3}^{(1)}-3 g F_{2}+4 g_{3}-4 E_{3}}{\pi t}-\frac{\Delta_{v} F_{2}^{2}}{8 \pi^{2} t}+\frac{2 F_{4}}{\pi^{2} t^{2}}+\mathcal{O}\left(e^{-2 \pi t}\right)
\end{aligned}
$$


and therefore, using the expansion of $\mathcal{Z}_{1}$ (in [32] (3.21)),

$$
\begin{aligned}
\mathcal{Z}_{4}= & -\frac{17 \pi^{2} t^{2}}{90}-\frac{\pi t}{3} g-6 E_{2}+\frac{F_{2}}{2}-\frac{g^{2}}{2}+\frac{4 D_{3}+4 D_{3}^{(1)}+8 E_{3}+7 g F_{2}-16\left(g_{3}+\zeta_{3}\right)}{2 \pi t} \\
& -\frac{\Delta_{v}\left(F_{2}^{2}+2 F_{4}\right)}{8 \pi^{2} t}-\frac{3 F_{2}^{2}-4 F_{4}+2 \mathcal{K}^{c}}{8 \pi^{2} t^{2}}+\mathcal{O}\left(e^{-2 \pi t}\right)
\end{aligned}
$$

where $\mathcal{K}^{c}$ is the regularized integral defined in formula (3.40) of [32].

\section{C.1.4 Non-separating degeneration of $\mathcal{Z}_{5}$}

To evaluate the non-separating asymptotics of $\mathcal{Z}_{5}$, we start from its defining formula in (3.14) as well as a closely related integral we shall denote here by $\mathcal{Z}_{5}^{\prime}$,

$$
\begin{aligned}
\mathcal{Z}_{5} & =\frac{16}{i \pi} \int_{\Sigma^{4}} \partial_{1} \mathcal{G}(1,2) \bar{\partial}_{1} \mathcal{G}(1,3) \mathcal{G}(1,4) \nu(2,4) \nu(4,3) \nu(3,2) \\
\mathcal{Z}_{5}^{\prime} & =\frac{16}{i \pi} \int_{\Sigma^{4}} \partial_{1} \mathcal{G}(1,2) \bar{\partial}_{1} \mathcal{G}(1,4) \mathcal{G}(1,3) \nu(2,4) \nu(4,3) \nu(3,2)
\end{aligned}
$$

Since this is the first example, here and in [32], of the non-separating degeneration of a genus-two modular graph functions which involves derivatives of the Arakelov Green function, we shall present the computations in detail. We begin by using the identity,

$$
\begin{aligned}
& \nu(x, y) \nu(y, z) \nu(z, x)+\nu(x, z) \nu(z, y) \nu(y, x) \\
& =-8 \kappa(x) \kappa(y) \kappa(z)-2 \kappa(x) \nu(y, z) \nu(z, y) \\
& \quad-2 \kappa(y) \nu(x, z) \nu(z, x)-2 \kappa(z) \nu(x, y) \nu(y, x)
\end{aligned}
$$

and the fact that the Arakelov Green function $\mathcal{G}(x, y)$ integrates to zero against the canonical Kähler form $\kappa(x)$, to conclude right away that we have,

$$
\mathcal{Z}_{5}+\mathcal{Z}_{5}^{\prime}=0
$$

To evaluate the difference, we instead use the identity,

$$
\begin{aligned}
& \nu(x, y) \nu(y, z) \nu(z, x)-\nu(x, z) \nu(z, y) \nu(y, x) \\
& \quad=\frac{\kappa(x)-\kappa_{1}(x)}{2 t \tau_{2}}\left(\omega_{t}(y) \bar{\omega}_{1}(y) \omega_{1}(z) \bar{\omega}_{t}(z)-\omega_{1}(y) \bar{\omega}_{t}(y) \omega_{t}(z) \bar{\omega}_{1}(z)\right)+\operatorname{cycl}(x, y, z)
\end{aligned}
$$

This leads to

$$
\begin{aligned}
\mathcal{Z}_{5}= & \frac{4 i}{\pi \tau_{2} t} \int_{\Sigma^{4}} \partial_{1} \mathcal{G}(1,2) \bar{\partial}_{1} \mathcal{G}(1,3) \mathcal{G}(1,4)\left(\kappa_{1}(2) \omega_{t}(3) \bar{\omega}_{1}(3) \omega_{1}(4) \bar{\omega}_{t}(4)\right. \\
& \left.-\kappa_{1}(2) \omega_{1}(3) \bar{\omega}_{t}(3) \omega_{t}(4) \bar{\omega}_{1}(4)+\operatorname{cycl}(2,3,4)\right)
\end{aligned}
$$

For fixed $z_{1}$, the integrals over $z_{2}, z_{3}, z_{4}$ reduce to one of the following integrals,

$$
\begin{gathered}
K(x)=\int_{\Sigma_{a b}} \kappa_{1}(y) \mathcal{G}(x, y)=\frac{\pi t}{12}-\frac{1}{4}\left(g\left(x, p_{a}\right)+g\left(x, p_{b}\right)-g\left(p_{a}, p_{b}\right)\right)+\frac{f(x)^{2}}{16 \pi t}+\mathcal{O}\left(e^{-2 \pi t}\right) \\
L(x)=\int_{\Sigma_{a b}} \omega_{t}(y) \bar{\omega}_{1}(y) \mathcal{G}(x, y)=\frac{\tau_{2}}{\pi}\left(\partial_{x} f_{2}(x)-\frac{1}{4} \partial_{p_{a}} f_{2}\left(p_{a}\right)-\frac{1}{4} \partial_{p_{b}} f_{2}\left(p_{b}\right)\right)+\mathcal{O}\left(e^{-2 \pi t}\right)(\mathrm{C} .
\end{gathered}
$$


to their complex conjugate, or to one of their derivatives,

$$
\begin{aligned}
& \partial_{x} K(x)=\int_{\Sigma_{a b}} \kappa_{1}(y) \partial_{x} \mathcal{G}(x, y)=-\frac{1}{4} \partial_{x} g\left(x, p_{a}\right)-\frac{1}{4} \partial_{x} g\left(x, p_{b}\right)+\frac{f(x) \partial_{x} f(x)}{8 \pi t}+\mathcal{O}\left(e^{-2 \pi t}\right) \\
& \partial_{x} L(x)=\int_{\Sigma_{a b}} \omega_{t}(y) \bar{\omega}_{1}(y) \partial_{x} \mathcal{G}(x, y)=\frac{\tau_{2}}{\pi} \partial_{x}^{2} f_{2}(x)+\mathcal{O}\left(e^{-2 \pi t}\right) \\
& \partial_{\bar{x}} L(x)=\int_{\Sigma_{a b}} \omega_{t}(y) \bar{\omega}_{1}(y) \partial_{\bar{x}} \mathcal{G}(x, y)=\frac{\tau_{2}}{\pi} \partial_{\bar{x}} \partial_{x} f_{2}(x)+\mathcal{O}\left(e^{-2 \pi t}\right)=-f(x)+\mathcal{O}\left(e^{-2 \pi t}\right)
\end{aligned}
$$

The function $f_{2}$ and its derivatives $\partial f_{2}, \bar{\partial} f_{2}$ are regular at $p_{a}, p_{b}$, while the singularities of $f, K, \partial^{2} f_{2}, \bar{\partial}^{2} f_{2}$ are powers of logarithms at the worst. As a result, the above integrals are absolutely convergent term by term and can be extended to the compact torus $\Sigma_{1}$,

$$
\begin{aligned}
\mathcal{Z}_{5}= & \frac{8}{\pi t} \int_{\Sigma_{1}} \kappa_{1}(x)\left[\partial_{x} K(x)\left(L(x) \partial_{\bar{x}} L^{*}(x)-L^{*}(x) \partial_{\bar{x}} L(x)\right)\right. \\
& +\partial_{\bar{x}} K(x)\left(L^{*}(x) \partial_{x} L(x)-L(x) \partial_{x} L^{*}(x)\right) \\
& \left.+K(x)\left(\bar{\partial}_{x} L^{*}(x) \partial_{\bar{x}} L(x)-\partial_{x} L(x) \partial_{\bar{x}} L^{*}(x)\right)\right]
\end{aligned}
$$

Integrating the first two lines by part so as to expose $K$ without derivatives, we get,

$$
\mathcal{Z}_{5}=\frac{24 \tau_{2}^{2}}{\pi^{3} t} \int_{\Sigma_{1}} \kappa_{1}(x) K(x)\left(\left(\partial_{x} \partial_{\bar{x}} f_{2}(x)\right)^{2}-\partial_{x}^{2} f_{2}(x) \partial_{\bar{x}}^{2} f_{2}(x)\right)+\mathcal{O}\left(e^{-2 \pi t}\right)
$$

Since we have,

$$
\left(\partial_{x} \partial_{\bar{x}} f_{2}(x)\right)^{2}-\partial_{x}^{2} f_{2}(x) \partial_{\bar{x}}^{2} f_{2}(x)=\frac{1}{2} \partial_{x}\left(\partial_{x} \partial_{\bar{x}} f_{2} \partial_{\bar{x}} f_{2}-\partial_{\bar{x}}^{2} f_{2} \partial_{x} f_{2}\right)+\text { c.c. }
$$

the $x$-independent terms in $K(x)$ give a vanishing contribution. The remaining terms may be organized as follows,

$$
\mathcal{Z}_{5}=\frac{C_{1}}{2 \pi t}+\frac{C_{2}}{2 \pi^{2} t^{2}}+\mathcal{O}\left(e^{-2 \pi t}\right)
$$

where $C_{1}$ and $C_{2}$ are $t$-independent genus-one elliptic modular functions, defined by,

$$
\begin{aligned}
& C_{1}=-12 \int_{\Sigma_{1}} \kappa_{1}(x)\left(g\left(x-p_{a}\right)+g\left(x-p_{b}\right)\right)\left(f(x)^{2}-\frac{\tau_{2}^{2}}{\pi^{2}} \partial_{x}^{2} f_{2} \partial_{\bar{x}}^{2} f_{2}\right) \\
& C_{2}=3 \int_{\Sigma_{1}} \kappa_{1}(x) f(x)^{2}\left(f(x)^{2}-\frac{\tau_{2}^{2}}{\pi^{2}} \partial_{x}^{2} f_{2} \partial_{\bar{x}}^{2} f_{2}\right)
\end{aligned}
$$

Using formulas (B.33) and (B.35) of [32], in particular,

$$
\partial_{x}^{2} f_{2}(x)=2 \pi i \partial_{\tau} f(x) \quad \partial_{\tau} \partial_{\bar{\tau}} g(x, y)=0
$$

it is immediate to compute $C_{2}$,

$$
C_{2}=72 F_{4}(v)-6 \Delta_{\tau} F_{4}(v)
$$


The integral of the term proportional to $f(x)^{2}$ in $C_{1}$ is easily evaluated, leading to,

$$
\begin{aligned}
C_{1} & =-24\left(D_{3}-D_{3}^{(1)}(v)\right)+24 \mathcal{C}_{1} \\
\mathcal{C}_{1} & =\frac{\tau_{2}^{2}}{\pi^{2}} \int_{\Sigma_{1}} \kappa_{1}(x) g\left(x, p_{a}\right) \partial_{x}^{2} f_{2} \partial_{\bar{x}}^{2} f_{2}
\end{aligned}
$$

The integral $\mathcal{C}_{1}$ is computed by integrating by parts and using $\partial_{x} \partial_{\bar{x}} f_{2}(x)=-\frac{\pi}{\tau_{2}} f(x)$,

$$
\begin{aligned}
\mathcal{C}_{1} & =-\frac{\tau_{2}^{2}}{\pi^{2}} \int_{\Sigma_{1}} \kappa_{1}(x)\left(\partial_{\bar{x}} g\left(x, p_{a}\right) \partial_{x}^{2} f_{2} \partial_{\bar{x}} f_{2}-\frac{\pi}{\tau_{2}} g\left(x, p_{a}\right) \partial_{x} f \partial_{\bar{x}} f_{2}\right) \\
& =\tilde{\mathcal{C}}_{1}-\frac{\tau_{2}}{\pi}\left|\partial f_{2}\left(p_{a}\right)\right|^{2}+2 E_{3}-2 g_{3}-D_{3}+D_{3}^{(1)}
\end{aligned}
$$

where

$$
\tilde{\mathcal{C}}_{1}=\frac{\tau_{2}}{\pi} \int_{\Sigma_{1}} \kappa_{1}(x) g\left(x, p_{a}\right)\left(\partial_{\bar{x}} f_{2} \partial_{x} f+\partial_{x} f_{2} \partial_{\bar{x}} f\right)
$$

To evaluate the last integral, we substitute $f=g\left(x, p_{b}\right)-g\left(x, p_{a}\right)$ and integrate by parts,

$$
\tilde{\mathcal{C}}_{1}=D_{3}-D_{3}^{(1)}(v)+\frac{\tau_{2}}{\pi} \int_{\Sigma_{1}} \kappa_{1}(x) g\left(x, p_{a}\right)\left(\partial_{\bar{x}} f_{2} \partial_{x} g\left(x, p_{b}\right)+\partial_{x} f_{2} \partial_{\bar{x}} g\left(x, p_{b}\right)\right)
$$

To compute this last integral, we use the following identity,

$$
\begin{aligned}
\partial_{x} \partial_{\bar{x}}\left(f_{2}(x) g\left(x, p_{b}\right)\right)= & \partial_{\bar{x}} f_{2} \partial_{x} g\left(x, p_{b}\right)+\partial_{x} f_{2} \partial_{\bar{x}} g\left(x, p_{b}\right) \\
& -\frac{\pi}{\tau_{2}}\left(f(x) g\left(x, p_{b}\right)+f_{2}(x)\left(\delta\left(x, p_{b}\right)-1\right)\right)
\end{aligned}
$$

The integral is now readily evaluated and we obtain,

$$
\begin{aligned}
& \tilde{\mathcal{C}}_{1}=D_{3}-D_{3}^{(1)}+2 g F_{2}+2 E_{3}-2 g_{3} \\
& \mathcal{C}_{1}=2 g F_{2}+4 E_{3}-4 g_{3}-\frac{\tau_{2}}{\pi}\left|\partial f_{2}\left(p_{a}\right)\right|^{2}
\end{aligned}
$$

Using $\tau_{2}\left|\partial f_{2}\left(p_{a}\right)\right|^{2}=\frac{1}{8 \pi} \Delta_{v}\left(F_{2}^{2}\right)-g F_{2}$ we find,

$$
C_{1}=-24\left(D_{3}-D_{3}^{(1)}\right)+72 g F_{2}+96\left(E_{3}-g_{3}\right)-\frac{3}{\pi} \Delta_{v} F_{2}^{2}
$$

We conclude that in the minimal non-separating degeneration,

$$
\mathcal{Z}_{5}=\frac{C_{1}}{2 \pi t}+\frac{C_{2}}{2 \pi^{2} t^{2}}+\mathcal{O}\left(e^{-2 \pi t}\right)
$$

where $C_{1}$ and $C_{2}$ are given by (C.50) and (C.43).

\section{C.1.5 A novel identity for genus-one elliptic modular graph functions}

Using the large $t$ expansions (C.16) and (C.29), and choosing coefficients judiciously, we observe that all terms up to order $\mathcal{O}\left(1 / t^{2}\right)$ cancel in the linear combination,

$$
\mathcal{Z}_{1}+\mathcal{Z}_{2}+\mathcal{Z}_{3}+\frac{1}{2} \mathcal{Z}_{4}-\varphi^{2}=\frac{\Delta_{\tau}\left(F_{2}^{2}-2 F_{4}\right)-6 F_{2}^{2}+4 F_{4}}{8 \pi^{2} t^{2}}+\mathcal{O}\left(e^{-2 \pi t}\right)
$$


Moreover, using the Laurent expansions computed in ([32], section C.3.3), we find that the coefficient of the $\mathcal{O}\left(1 / t^{2}\right)$ in (C.52) is exponentially suppressed near the cusp. This strongly suggests that the left-hand side of (C.52) actually vanishes, motivating the conjectures (3.15) and (3.16). In appendix D, we shall prove that the genus-two identity (3.15) indeed holds, and obtain (3.16) as a consequence of this fact. ${ }^{25}$ Without doubt, (3.16) is only the first in an infinite family of relations between genus-one elliptic MGFs, and systematic methods for deriving such identities are being developed in [92].

\section{C.2 Separating degeneration}

We shall now consider the separating degeneration, where the genus-two Riemann surface $\Sigma$ degenerates into two genus-one curves $\Sigma_{1}$ and $\Sigma_{1}^{\prime}$, with two marked points $p \in \Sigma_{1}, p^{\prime} \in$ $\Sigma_{1}^{\prime}$ joined by a thin tube. We refer to ([32], section 4) for a detailed discussion of this degeneration, and only recall a few basic facts.

This limit is obtained by sending to zero the off-diagonal entry of the period matrix $\Omega$, keeping fixed the diagonal entries $\tau, \sigma$ corresponding to the complex moduli of $\Sigma_{1}$ and $\Sigma_{1}^{\prime}$. In the limit $v \rightarrow 0$, the Siegel modular group $\operatorname{Sp}(4, \mathbb{Z})$ is broken to the product $\operatorname{SL}(2, \mathbb{Z})_{\tau} \times$ $\operatorname{SL}(2, \mathbb{Z})_{\sigma} \ltimes \mathbb{Z}_{2}$, where the two $\mathrm{SL}(2, \mathbb{Z})$ factors act by fractional linear transformations of $\tau$ and $\sigma$ and $\mathbb{Z}_{2}$ exchanges these two variables. The modulus $|\hat{v}|$ of the degeneration parameter

$$
\hat{v}=2 \pi v \eta(\tau)^{2} \eta(\sigma)^{2}
$$

stays invariant under the unbroken part of $\operatorname{Sp}(4, \mathbb{Z})$. The Abelian differentials degenerate, up to terms of order $\mathcal{O}(|\hat{v}|)$, to

$$
\omega_{1}=\left\{\begin{array}{ll}
\omega(x) & x \in \Sigma_{1} \\
0 & x \in \Sigma_{1}^{\prime}
\end{array}, \quad \omega_{2}= \begin{cases}0 & x \in \Sigma_{1} \\
\omega^{\prime}(x) & x \in \Sigma_{1}^{\prime}\end{cases}\right.
$$

where $\omega$ and $\omega^{\prime}$ are the standard Abelian differentials on $\Sigma_{1}$ and $\Sigma_{1}^{\prime}$. The Arakelov Green function becomes, up to terms of order $\mathcal{O}(|\hat{v}|)$,

$$
\mathcal{G}(x, y) \sim \begin{cases}-\frac{1}{2} \ln |\hat{v}|+g(x-y \mid \tau)-\frac{1}{2} g(x-p \mid \tau)-\frac{1}{2} g(y-p \mid \tau) & x, y \in \Sigma_{1} \\ -\frac{1}{2} \ln |\hat{v}|+g(x-y \mid \sigma)-\frac{1}{2} g\left(x-p^{\prime} \mid \sigma\right)-\frac{1}{2} g\left(y-p^{\prime} \mid \sigma\right) & x, y \in \Sigma_{1}^{\prime} \\ \frac{1}{2} \ln |\hat{v}|+\frac{1}{2} g(x-p \mid \tau)+\frac{1}{2} g\left(y-p^{\prime} \mid \sigma\right), & x \in \Sigma_{1}, y \in \Sigma_{1}^{\prime}\end{cases}
$$

The expansion of the modular graph functions $\varphi$ and $\mathcal{Z}_{1,2,3}$ was computed in ([32], section 4) using these formulae,

$$
\begin{aligned}
\varphi & =-\ln |\hat{v}|+\mathcal{O}(|\hat{v}|) \\
\mathcal{Z}_{1} & =2(\ln |\hat{v}|)^{2}+4 E_{2}(\tau)+4 E_{2}(\sigma)+\mathcal{O}(|\hat{v}|) \\
\mathcal{Z}_{2} & =-2(\ln |\hat{v}|)^{2}-E_{2}(\tau)-E_{2}(\sigma)+\mathcal{O}(|\hat{v}|) \\
\mathcal{Z}_{3} & =2(\ln |\hat{v}|)^{2}+\mathcal{O}(|\hat{v}|)
\end{aligned}
$$

where $E_{k}(\tau)$ is the usual non-holomorphic Eisenstein series (4.57) of $\operatorname{SL}(2, \mathbb{Z})$.

\footnotetext{
${ }^{25}$ After the first version of this work, a direct proof of (3.16) based on genus-one methods has been given in $[72]$.
} 
For the integral $\mathcal{Z}_{4}$ defined in (3.14), we see that the measure $\nu(x, y) \nu(y, x)$ vanishes in the limit $v \rightarrow 0$ unless $x, y$ lie on the same elliptic curve, say $\Sigma_{1}$, in which case it reduces to $-\kappa_{1}(x) \kappa_{1}(y)$. Hence the integral reduces to

$$
-4 \int_{\Sigma_{1}^{4}}\left[-\frac{1}{2} \ln |\hat{v}|+g(x-y \mid \tau)-\frac{1}{2} g(x-p \mid \tau)-\frac{1}{2} g(y-p \mid \tau)\right]^{2} \kappa_{1}(x) \kappa_{1}(y)+\left(\Sigma_{1} \leftrightarrow \Sigma_{1}^{\prime}\right)
$$

Observing that the crossproducts integrate to zero, this evaluates to

$$
\begin{aligned}
\mathcal{Z}_{4} & \sim-\int_{\Sigma_{1}}\left[(\ln |\hat{v}|)^{2}+4 g(x-y \mid \tau)^{2}+g(x-p \mid \tau)^{2}+g(y-p \mid \tau)^{2}\right] \kappa_{1}(x) \kappa_{1}(y)+\left(\Sigma_{1} \leftrightarrow \Sigma_{1}^{\prime}\right) \\
& =-2(\ln |\hat{v}|)^{2}-6 E_{2}(\tau)-6 E_{2}(\sigma)+\mathcal{O}(|\hat{v}|)
\end{aligned}
$$

Using (C.56) this behavior is indeed consistent with the identity (3.15).

Turning to the integral $\mathcal{Z}_{5}$ defined in (3.14), we see that the measure $\nu(2,4) \nu(4,3) \nu(3,2)$ vanishes unless the points $2,3,4$ are on the same elliptic curve, say $\Sigma_{1}$, in which case it reduces to $\kappa_{1}(2) \kappa_{1}(3) \kappa_{1}(4)$. When the point 1 is also on $\Sigma_{1}$, we get

$$
\begin{aligned}
& \frac{16}{i \pi} \int_{\Sigma_{1}^{4}}\left[\partial_{1} g\left(x_{1}-x_{2}\right)-\frac{1}{2} \partial_{1} g\left(x_{1}-p\right)\right]\left[\bar{\partial}_{1} g\left(x_{1}-x_{3}\right)-\frac{1}{2} \bar{\partial}_{1} g\left(x_{1}-p\right)\right] \\
& \quad \times\left[-\frac{1}{2} \ln |\hat{v}|+g\left(x_{1}-x_{4}\right)-\frac{1}{2} g\left(x_{1}-p\right)-\frac{1}{2} g\left(x_{4}-p\right)\right] \kappa_{1}(2) \kappa_{1}(3) \kappa_{1}(4)
\end{aligned}
$$

In the first line, using translational invariance one can replace $\partial_{1} g\left(x_{1}-x_{2}\right)$ and $\bar{\partial}_{1} g\left(x_{1}-\right.$ $\left.x_{3}\right)$ by $-\partial_{2} g\left(x_{1}-x_{2}\right)$ and $-\bar{\partial}_{3} g\left(x_{1}-x_{3}\right)$, which integrate by parts to zero. The terms proportional to $g\left(x_{1}-x_{4}\right)$ or $g\left(x_{4}-p\right)$ on the second line also integrate to zero. The integrals over $x_{2}, x_{3}, x_{4}$ are then trivial, leading to

$$
-\frac{2}{i \pi} \int_{\Sigma_{1}} \partial_{1} g\left(x_{1}-p\right) \bar{\partial}_{1} g\left(x_{1}-p\right)\left[\ln |\hat{v}|+g\left(x_{1}-p\right)\right]
$$

When the point 1 is on $\Sigma_{1}^{\prime}$, we get instead

$$
\begin{aligned}
& \frac{4}{i \pi} \int_{\Sigma_{1}^{\prime} \times \Sigma_{1}^{3}} \partial_{1} g\left(x_{1}-p^{\prime}\right) \bar{\partial}_{1} g\left(x_{1}-p^{\prime}\right)\left[\frac{1}{2} \ln |\hat{v}|+\frac{1}{2} g\left(x_{1}-p^{\prime}\right)+\frac{1}{2} g\left(x_{4}-p\right)\right] \kappa_{1}(2) \kappa_{1}(3) \kappa_{1}(4) \\
& =\frac{2}{i \pi} \int_{\Sigma_{1}^{\prime}} \partial_{1} g\left(x_{1}-p^{\prime}\right) \bar{\partial}_{1} g\left(x_{1}-p^{\prime}\right)\left[\ln |\hat{v}|+g\left(x_{1}-p^{\prime}\right)\right]
\end{aligned}
$$

The contributions (C.60) and (C.61) cancel against those where $\Sigma_{1}$ and $\Sigma_{1}^{\prime}$ are exchanged, so we find that $\mathcal{Z}_{5}$ vanishes in the separating degeneration, up to terms of order $|\hat{v}|$. It is quite remarkable that $\mathcal{Z}_{5}$ vanishes both in the separating and non-separating degenerations.

\section{C.3 Tropical limit}

Having obtained the expansion of $\mathcal{Z}_{4}$ and $\mathcal{Z}_{5}$ in the non-separating degeneration $t \rightarrow \infty$ keeping fixed $\tau$ and $v$, we can obtain the tropical limit by further sending $\tau \rightarrow i \infty$ keeping 
$u_{2}=\operatorname{Im} v / \tau_{2}$ fixed. The result can be re-expressed in terms of the variables $V, S=S_{1}+i S_{2}$ parametrizing the imaginary part of the period matrix via,

$$
Y=\frac{1}{V S_{2}}\left(\begin{array}{cc}
1 & S_{1} \\
S_{1} & |S|^{2}
\end{array}\right)
$$

such that the tropical limit corresponds to $V=\left(t \tau_{2}\right)^{-1 / 2} \rightarrow 0$ keeping $S_{1}=u_{2}$ and $S_{2}=\left(t / \tau_{2}\right)^{1 / 2}$ fixed. For the modular graph functions $\varphi$ and $\mathcal{Z}_{1,2,3}$ in (3.14), this leads to $([32]$, section 5$)$,

$$
\begin{aligned}
\varphi \sim & \frac{5 \pi}{6 V} A_{1,0}+\frac{5 \zeta_{3}}{4 \pi^{2}} A_{0,0} V^{2} \\
\varphi^{2} \sim & \frac{32 \pi^{2}}{V}\left[-\frac{1}{1512} A_{0,0}+\frac{1}{1512} A_{0,2}-\frac{5}{1584} A_{1,1}+\frac{25}{1152} A_{2,0}\right]+\frac{25 \zeta_{3}}{12 \pi} A_{1,0} V+\frac{25 \zeta_{3}^{2}}{16 \pi^{4}} V^{4} \\
\mathcal{Z}_{1} \sim & \frac{32 \pi^{2}}{V^{2}}\left[-\frac{1}{315} A_{0,0}+\frac{1}{252} A_{0,2}-\frac{1}{792} A_{1,1}+\frac{23}{960} A_{2,0}\right] \\
& +\frac{\zeta_{3}}{\pi}\left[\frac{18}{5} A_{0,1}-\frac{1}{2} A_{1,0}\right] V-\frac{\zeta_{5}}{2 \pi^{3}} A_{0,1} V^{3}+\zeta_{3}^{2} \frac{(2 \beta-3)}{16 \pi^{4}} A_{0,0} V^{4} \\
\mathcal{Z}_{2} \sim & \frac{32 \pi^{2}}{V^{2}}\left[\frac{1}{504} A_{0,0}-\frac{1}{1008} A_{0,2}-\frac{5}{792} A_{1,1}-\frac{17}{960} A_{2,0}\right]-\frac{5 \zeta_{3}}{2 \pi} A_{1,0} V-\frac{7 \zeta_{5}}{4 \pi^{3}} A_{0,1} V^{3} \\
\mathcal{Z}_{3} \sim & \frac{32 \pi^{2}}{V^{2}}\left[-\frac{11}{7560} A_{0,0}+\frac{1}{1512} A_{0,2}+\frac{1}{792} A_{11}+\frac{17}{576} A_{2,0}\right]+\frac{5 \zeta_{3}}{6 \pi} A_{1,0} V+\frac{11 \zeta_{3}^{2}}{8 \pi^{4}} A_{0,0} V^{4}
\end{aligned}
$$

where $\sim$ indicates equality in the limit $V \rightarrow 0$ up to corrections of order $e^{-1 / V}$.

Similarly, starting with (C.29) and using the formulae in appendix C of [32], we arrive at

$$
\begin{aligned}
\mathcal{Z}_{4} \sim & \frac{32 \pi^{2}}{V^{2}}\left[\frac{1}{252} A_{0,0}-\frac{1}{168} A_{0,2}+\frac{5}{792} A_{1,1}-\frac{9}{320} A_{2,0}\right] \\
& +\zeta_{3} \frac{85 A_{1,0}-72 A_{0,1}}{10 \pi} V+\frac{9}{2 \pi^{3}} \zeta_{5} A_{0,1} V^{3}+\zeta_{3}^{2} \frac{3-\beta}{4 \pi^{4}} A_{0,0} V^{4}
\end{aligned}
$$

where $A_{i, j}(S)$ are the local modular forms introduced in section 5.3 of loc. cit., and $\beta$ is the unknown (presumably rational) coefficient appearing at order $V^{4}$ in the tropical limit of $\mathcal{Z}_{1}$. As a strong consistency check on the expansion (C.29), we have reproduced the leading term in (C.64) from a worldline integral using the tropical Arakelov Green function $\mathcal{G}^{(s g)}$ along the lines of ([32], section 5.3).

Using (C.64) we obtain the tropical limit of the combinations $A_{1}$ and $A_{2}$ defined in (4.6),

$$
\begin{aligned}
A_{1} \sim & \frac{32 \pi^{2}}{V^{2}}\left[-\frac{13}{1512} A_{0,0}+\frac{5}{756} A_{0,2}+\frac{5}{396} A_{1,1}+\frac{4}{45} A_{2,0}\right] \\
& +\zeta_{3}\left[\frac{18}{5 \pi} A_{0,1}+\frac{16}{3 \pi} A_{1,0}\right] V+\frac{3 \zeta_{5}}{\pi^{3}} A_{0,1} V^{3}+\frac{(2 \beta+13) \zeta_{3}^{2}}{16 \pi^{4}} V^{4} \\
A_{2} \sim & \frac{32 \pi^{2}}{V^{2}}\left[\frac{1}{7560} A_{0,0}+\frac{1}{1512} A_{0,2}-\frac{1}{132} A_{1,1}+\frac{1}{72} A_{2,0}\right]+\frac{10 \zeta_{3}}{3 \pi} A_{1,0} V+\frac{7 V^{4} \zeta_{3}^{2}}{4 \pi^{4}}
\end{aligned}
$$


In contrast, we find that the tropical limit of the integral (C.30) starts at order $V$,

$$
\mathcal{Z}_{5} \sim \frac{72}{\pi}\left(A_{1,0}-\frac{1}{5} A_{0,1}\right) V \zeta_{3}+\frac{30}{\pi^{3}} A_{0,1} V^{3} \zeta_{5}
$$

The vanishing of the leading $\mathcal{O}\left(1 / V^{2}\right)$ term is quite remarkable, and follows from the cancellation of the leading term in the combination $f(x)^{2}-\frac{\tau_{2}^{2}}{\pi^{2}} \partial_{x}^{2} f_{2} \partial_{\bar{x}}^{2} f_{2}$ appearing on both lines of (C.41). We have also confirmed the vanishing at leading order by a wordline computation using the tropical Arakelov Green function.

\section{Proof of the modular graph function identities}

In this subsection, we shall prove the identity (3.15) between the genus-two modular graph functions defined in (3.13) and (3.14).

Translation invariance and the resulting momentum conservation on the torus provides a fundamental tool in the proof of identities between genus-one modular functions (along with holomorphic subgraph reduction $[43,93]$ or Fay identities [94]). The absence of translation invariance prevents us from using the same techniques for higher genus surfaces. However, the lemma below provides the appropriate alternative tool, valid for arbitrary genus $h .^{26}$

Lemma D.1 On a compact Riemann surface $\Sigma$, with Arakelov Green function $\mathcal{G}(x, y)$, the following identity holds for arbitrary $y, z \in \Sigma$,

$$
\omega_{I}(y) \partial_{z} \mathcal{G}(y, z)+\omega_{I}(z) \partial_{y} \mathcal{G}(y, z)-\partial_{z} \Phi_{I}^{J}(z) \omega_{J}(y)-\partial_{y} \Phi_{I}^{J}(y) \omega_{J}(z)=0
$$

where the tensor $\Phi_{I}^{J}(z)=\Phi_{I K}(z) Y^{K J}$ is given by the following integral in $x \in \Sigma$,

$$
\Phi_{I J}(z)=\frac{i}{2} \int_{\Sigma} \mathcal{G}(z, x) \omega_{I}(x) \overline{\omega_{J}(x)}
$$

The tensor $\Phi$ is Hermitian $\overline{\Phi_{I J}(z)}=\Phi_{J I}(z)$.

To prove Lemma D.1, we first show that its left side is holomorphic in $z$ and thus holomorphic in $y$ by symmetry under swapping $z$ and $y$. The $\partial_{\bar{y}}$ derivative of the left side of (D.1) may be evaluated using the identities on the Arakelov Green function $\mathcal{G}$, given in (A.14) for genus 2. The $\delta(y, z)$-functions cancel between the first two terms, and $\Phi$ has been defined so as to cancel also the remaining terms in the $\partial_{\bar{y}}$ derivative. As a result, the left side of (D.1) is a single-valued holomorphic (1,0)-form in $z$ and $y$ which takes the form $M_{I} J K \omega_{J}(z) \omega_{K}(y)$ for some constant tensor $M_{I}^{J K}$. To show that $M=0$, we integrate the left side of (D.1) against $\bar{\omega}_{L}(z)$, use the fact that the contributions from the first and third terms vanish, and that those of the second and fourth terms cancel using the definition of $\Phi$.

The proof of the identity (3.15) proceeds by a judicious use of the formula (D.1). We begin by considering the alternative integral for $\mathcal{Z}_{2}$ given on the first line of (A.16),

$$
\mathcal{Z}_{2}=-4 \int_{\Sigma^{3}} \nu(x, y) \nu(y, x) \kappa(z) \mathcal{G}(x, z) \mathcal{G}(y, z)
$$

\footnotetext{
${ }^{26}$ Since the first version of this work, the lemma (D.1) has been further generalized and applied to derive higher-weight identities at arbitrary genus [71].
} 
and eliminate $\nu(x, y)$ defined by (3.11) using the second equation in (A.14). The $\delta$-function produces the term $-\mathcal{Z}_{1}$ on the right side, so that we obtain,

$$
\mathcal{Z}_{1}+\mathcal{Z}_{2}=\frac{2 i}{\pi} \int_{\Sigma^{3}} \nu(y, x) \partial_{x} \partial_{\bar{y}} \mathcal{G}(x, y) \mathcal{G}(x, z) \kappa(z) \mathcal{G}(z, y)
$$

Integrating by parts in $x$ and writing out $\kappa(z)$ explicitly, we obtain,

$$
\mathcal{Z}_{1}+\mathcal{Z}_{2}=\frac{Y^{I J}}{2 \pi} \int_{\Sigma^{3}} \nu(y, x) \omega_{I}(z) \bar{\omega}_{J}(z) \partial_{\bar{y}} \mathcal{G}(x, y) \partial_{x} \mathcal{G}(x, z) \mathcal{G}(z, y)
$$

Next, we use formula (D.1) to re-express the combination $\omega_{I}(z) \partial_{x} \mathcal{G}(x, z)$,

$$
\begin{aligned}
\mathcal{Z}_{1}+\mathcal{Z}_{2}= & \frac{Y^{I J}}{2 \pi} \int_{\Sigma^{3}} \nu(y, x) \bar{\omega}_{J}(z) \partial_{\bar{y}} \mathcal{G}(x, y) \mathcal{G}(z, y) \\
& \times\left[-\omega_{I}(x) \partial_{z} \mathcal{G}(z, x)+\partial_{z} \Phi_{I}{ }^{K}(z) \omega_{K}(x)+\partial_{x} \Phi_{I}{ }^{K}(x) \omega_{K}(z)\right]
\end{aligned}
$$

To evaluate the contributions from the first two terms inside the brackets, we integrate by parts in both $\bar{y}$ and $z$ and combine various Abelian differentials into $\nu(x, z)$. For the first term we obtain,

$$
-\frac{Y^{I J}}{2 \pi} \int_{\Sigma^{3}} \nu(y, x) \bar{\omega}_{J}(z) \mathcal{G}(x, y) \partial_{z} \partial_{\bar{y}} \mathcal{G}(z, y) \omega_{I}(x) \mathcal{G}(z, x)=-\frac{1}{2} \mathcal{Z}_{4}+\frac{1}{2} \mathcal{Z}_{2}
$$

where we have used the second equation in (A.14) for the mixed double derivative on $\mathcal{G}$, the expression for $\mathcal{Z}_{4}$ in (3.14) to evaluate the contribution from the $\delta$-function, and the alternative formula for $\mathcal{Z}_{2}$ given in the second line of (A.16). For the second term we obtain,

$$
\begin{aligned}
& -i Y^{I J} \int_{\Sigma^{2}} \nu(y, x) \bar{\omega}_{J}(y) \omega_{K}(x) \mathcal{G}(x, y) \Phi_{I}{ }^{K}(y) \\
& +i Y^{I J} \int_{\Sigma^{3}} \nu(y, x) \bar{\omega}_{J}(z) \omega_{K}(x) \nu(z, y) \mathcal{G}(x, y) \Phi_{I}{ }^{K}(z)
\end{aligned}
$$

Using the formula (D.2) for $\Phi$ makes all Abelian differentials explicit, and regrouping these into $\nu$ differentials we obtain for the second term inside the brackets of (D.6),

$$
-2 \int_{\Sigma^{3}} \nu(x, z) \nu(z, y) \nu(y, x) \mathcal{G}(x, y) \mathcal{G}(y, z)=\frac{1}{2} \mathcal{Z}_{2}+\varphi^{2}
$$

where we have used the alternative integral for $\mathcal{Z}_{2}$ in the second line of (A.16) as well as the formula for $\varphi^{2}$ on the third line of (A.16). Finally, to evaluate the contribution from the third term inside the brackets of (D.6), we integrate by parts in $x$ and again use the second equation in (A.14) to obtain,

$$
\begin{aligned}
& i Y^{I J} \int_{\Sigma^{2}} \nu(x, x) \bar{\omega}_{J}(z) \omega_{K}(x) \mathcal{G}(x, z) \Phi_{I}^{K}(x) \\
& -i Y^{I J} \int_{\Sigma^{3}} \nu(y, x) \bar{\omega}_{J}(z) \omega_{K}(z) \nu(z, y) \mathcal{G}(z, y) \Phi_{I}{ }^{K}(x)
\end{aligned}
$$


Using the formula (D.2) for $\Phi$ makes all Abelian differentials explicit, and regrouping these into $\nu$ differentials we obtain for the third term inside the brackets of (D.6),

$$
\begin{aligned}
& 4 \int_{\Sigma^{3}} \kappa(x) \nu(z, y) \nu(y, z) \mathcal{G}(x, y) \mathcal{G}(x, z) \\
& -2 \int_{\Sigma^{4}} \nu(x, y) \nu(y, x) \nu(z, w) \nu(w, z) \mathcal{G}(x, w) \mathcal{G}(y, z) \\
& \quad=-\mathcal{Z}_{2}-\mathcal{Z}_{3}
\end{aligned}
$$

where we have used the second line of (A.16) to evaluate the first integral, and (A.8) twice to transform the second integral into the expression for $-\mathcal{Z}_{3}$ with $\mathcal{Z}_{3}$ given in (3.14). Assembling all contributions proves formula (3.15).

By evaluating the non-separating asymptotics of the function $\mathcal{Z}_{4}$-function independently and using the asymptotics obtained for $\varphi, \mathcal{Z}_{1}, \mathcal{Z}_{2}, \mathcal{Z}_{3}$ in [32], we have shown in appendix C.1.5 that (3.15) implies a highly non-trivial identity between the genus-one elliptic modular graph functions $F_{2}$ and $F_{4}$.

\section{E Overall normalization of the genus-two amplitude}

The five-point genus-two amplitude including its overall coefficient was determined in [8] at the leading order $D^{2} \mathcal{R}^{5}$ using the non-minimal pure spinor formalism. The normalization of the amplitude followed from a first principles calculation using the integrals over pure spinor space derived in [73] together with the conventions for genus-two measures of [57]. Consequently, the building blocks $T_{1,2,3 \mid 4,5}^{m}$ and $T_{12,3 \mid 4,5}$ (collectively denoted by $T^{\mathrm{NMPS}}$ ) featured in [8] also depend on zero-modes of the non-minimal pure spinor $\bar{\lambda}_{\alpha}$ whose integration gives rise to various combinatorial factors.

In contrast, in this work we use the building blocks $T_{1,2,3 \mid 4,5}^{m}$ and $T_{12,3 \mid 4,5}$ (collectively denoted by $T^{\mathrm{MPS}}$ ) defined with the minimal pure spinor formalism in [14]; which do not depend on the zero-modes of $\bar{\lambda}_{\alpha}$. Despite their different definitions, one can verify that BRST-invariant quantities written in either setup yield the same results with differing normalizations. The component expansion for bosonic external states of the building blocks used in this work can be downloaded from [63]. We will now show that their relative normalization is such that $T^{\mathrm{NMPS}}=2^{10} 3^{3} 5 T^{\mathrm{MPS}}$.

To show this we compare the component expansion of the BRST-invariant kinematic factor at order $D^{2} \mathcal{R}^{5}$. The pure spinor superspace representation $\left.\mathcal{B}_{(5)}\right|_{D^{2} \mathcal{R}^{5}}$ obtained in this work (4.19) coincides with equation (5.44) from [8],

$$
\begin{aligned}
\mathcal{K}_{5}^{(2)}= & \frac{\left|\left\langle T_{12,3 \mid 4,5}\right\rangle_{0}\right|^{2}}{s_{12}}+\frac{\left|\left\langle T_{12,4 \mid 3,5}\right\rangle_{0}\right|^{2}}{s_{12}}+\frac{\left|\left\langle T_{12,5 \mid 3,4}\right\rangle_{0}\right|^{2}}{s_{12}} \\
& +\left|\left\langle T_{3,4,5 \mid 1,2}^{m}\right\rangle_{0}\right|^{2}+(1,2 \mid 1,2,3,4,5)
\end{aligned}
$$

up to an overall coefficient, ${ }^{27}$

$$
\left.\mathcal{B}_{(5)}\right|_{D^{2} \mathcal{R}^{5}}=2^{6}\left(\frac{\alpha^{\prime}}{2}\right) \mathcal{K}_{5}^{(2)}
$$

\footnotetext{
${ }^{27}$ We note the different convention for Mandelstam invariants, where $s_{i j}^{\text {here }}=\left(\alpha^{\prime} / 2\right) s_{i j}^{\text {there }}$.
} 
Straightforward calculations for 5 Type IIB gravitons show that

$$
\begin{aligned}
\left.\mathcal{B}_{(5)}\right|_{D^{2} \mathcal{R}^{5}} & =-2^{14}\left(\frac{2}{\alpha^{\prime}}\right)^{4} \mathcal{B}_{\{5\}}^{\text {tree }} \\
\left.\mathcal{K}_{5}^{(2)}\right|_{\text {IIB }} & =-2^{28} 3^{6} 5^{2}\left(\frac{2}{\alpha^{\prime}}\right)^{5} \mathcal{B}_{\{5\}}^{\text {tree }}
\end{aligned}
$$

where the result in the non-minimal formalism is given in equation (5.46) of [8]. From (E.2) and (E.3) it follows that $T^{\mathrm{NMPS}}=2^{10} 3^{3} 5 T^{\mathrm{MPS}}$.

It remains to explain the overall coefficient of the genus-two five-point amplitude in (4.49). It matches the normalization of the amplitude derived in equations (5.41) and (5.43) of $[8]$,

$$
\mathcal{A}_{(5)}^{2-\text { loop }}=(2 \pi)^{10} \delta^{10}(k)\left(\frac{\alpha^{\prime}}{2}\right)^{5} \frac{\kappa^{5} e^{2 \lambda}}{2^{45} 3^{6} 5^{2} \pi^{6}} \int_{\mathcal{M}_{2}} \frac{\left|d^{3} \Omega\right|^{2}}{(\operatorname{det} Y)^{5}} \int_{\Sigma^{5}}\left|\left\langle\mathcal{K}^{(2)}\left(z_{1}, \ldots, z_{5}\right)\right\rangle_{0}\right|^{2} \mathrm{KN}_{(5)}
$$

where the integral over vertex points is given by,

$$
\int_{\Sigma_{5}}\left|\left\langle\mathcal{K}^{(2)}\left(z_{1}, \ldots, z_{5}\right)\right\rangle_{0}\right|^{2} \mathrm{KN}_{(5)}=2^{6} \pi\left(\frac{\alpha^{\prime}}{2}\right)(\operatorname{det} Y)^{2} \mathcal{K}_{5}^{(2)}+\mathcal{O}\left(\alpha^{\prime 2}\right)
$$

Equation (E.5) is the origin of the different factor of $2^{6}\left(\alpha^{\prime} / 2\right)$ in (E.2), while the factor of $\pi$ is taken into account in the normalization of (4.49) which contains $1 / \pi^{5}$ instead of $1 / \pi^{6}$ in (E.4). ${ }^{28}$

The precise normalization of the five-point SYM tree amplitude used in section 4.5 follows from the evaluation of $A_{\mathrm{YM}}(1,2,3,4,5)=\left\langle E_{1234} V_{5}\right\rangle_{0}$ [80] with the measure normalized as $\left\langle\left(\lambda \gamma^{m} \theta\right)\left(\lambda \gamma^{n} \theta\right)\left(\lambda \gamma^{p} \theta\right)\left(\theta \gamma_{m n p} \theta\right)\right\rangle_{0}=1$. The five-point tree amplitude available in [63] is 2880 times bigger.

Open Access. This article is distributed under the terms of the Creative Commons Attribution License (CC-BY 4.0), which permits any use, distribution and reproduction in any medium, provided the original author(s) and source are credited.

\section{References}

[1] E. D'Hoker and D.H. Phong, The Geometry of String Perturbation Theory, Rev. Mod. Phys. 60 (1988) 917 [INSPIRE].

[2] E. D'Hoker and D.H. Phong, Lectures on two loop superstrings, Conf. Proc. C 0208124 (2002) 85 [hep-th/0211111] [INSPIRE].

[3] E. Witten, Notes On Super Riemann Surfaces And Their Moduli, Pure Appl. Math. Quart. 15 (2019) 57 [arXiv:1209.2459] [INSPIRE].

[4] E. Witten, Superstring Perturbation Theory Revisited, arXiv:1209.5461 [INSPIRE].

[5] M.B. Green, J.H. Schwarz and L. Brink, $N=4$ Yang-Mills and $N=8$ Supergravity as Limits of String Theories, Nucl. Phys. B 198 (1982) 474 [inSPIRE].

\footnotetext{
${ }^{28}$ In general, this difference is taken into account by the factor $\frac{1}{\pi}$ in the definition of (2.49).
} 
[6] E. D'Hoker and D.H. Phong, Two-loop superstrings VI: Non-renormalization theorems and the 4-point function, Nucl. Phys. B 715 (2005) 3 [hep-th/0501197] [INSPIRE].

[7] N. Berkovits, Super-Poincaré covariant two-loop superstring amplitudes, JHEP 01 (2006) 005 [hep-th/0503197] [INSPIRE].

[8] H. Gomez, C.R. Mafra and O. Schlotterer, Two-loop superstring five-point amplitude and S-duality, Phys. Rev. D 93 (2016) 045030 [arXiv: 1504.02759] [InSPIRE].

[9] H. Gomez and C.R. Mafra, The closed-string 3-loop amplitude and S-duality, JHEP 10 (2013) 217 [arXiv:1308.6567] [INSPIRE].

[10] N. Berkovits, Pure spinor formalism as an $N=2$ topological string, JHEP 10 (2005) 089 [hep-th/0509120] [INSPIRE].

[11] E. D'Hoker and D.H. Phong, Conformal Scalar Fields and Chiral Splitting on SuperRiemann Surfaces, Commun. Math. Phys. 125 (1989) 469 [INSPIRE].

[12] E. D'Hoker, C.R. Mafra, B. Pioline and O. Schlotterer, Two-loop superstring five-point amplitudes. Part I. Construction via chiral splitting and pure spinors, JHEP 08 (2020) 135 [arXiv: 2006. 05270] [INSPIRE].

[13] J.J.M. Carrasco and H. Johansson, Five-Point Amplitudes in $N=4$ Super-Yang-Mills Theory and $N=8$ Supergravity, Phys. Rev. D 85 (2012) 025006 [arXiv:1106.4711] [INSPIRE].

[14] C.R. Mafra and O. Schlotterer, Two-loop five-point amplitudes of super Yang-Mills and supergravity in pure spinor superspace, JHEP 10 (2015) 124 [arXiv:1505. 02746] [INSPIRE].

[15] E. D'Hoker and O. Schlotterer, Two-loop superstring five-point amplitudes III, construction via the RNS formulation, to appear.

[16] M.B. Green and M. Gutperle, Effects of D instantons, Nucl. Phys. B 498 (1997) 195 [hep-th/9701093] [INSPIRE].

[17] M.B. Green and S. Sethi, Supersymmetry constraints on type IIB supergravity, Phys. Rev. D 59 (1999) 046006 [hep-th/9808061] [INSPIRE].

[18] M.B. Green, H.-h. Kwon and P. Vanhove, Two loops in eleven-dimensions, Phys. Rev. D 61 (2000) 104010 [hep-th/9910055] [INSPIRE].

[19] M.B. Green and P. Vanhove, Duality and higher derivative terms in M-theory, JHEP 01 (2006) 093 [hep-th/0510027] [INSPIRE].

[20] M.B. Green, S.D. Miller and P. Vanhove, $S L(2, \mathbb{Z})$-invariance and D-instanton contributions to the $D^{6} R^{4}$ interaction, Commun. Num. Theor. Phys. 09 (2015) 307 [arXiv:1404.2192] [INSPIRE].

[21] M.B. Green, J.G. Russo and P. Vanhove, Automorphic properties of low energy string amplitudes in various dimensions, Phys. Rev. D 81 (2010) 086008 [arXiv:1001.2535] [INSPIRE].

[22] M.B. Green, S.D. Miller, J.G. Russo and P. Vanhove, Eisenstein series for higher-rank groups and string theory amplitudes, Commun. Num. Theor. Phys. 4 (2010) 551 [arXiv: 1004.0163] [INSPIRE].

[23] G. Bossard, A. Kleinschmidt and B. Pioline, 1/8-BPS Couplings and Exceptional Automorphic Functions, SciPost Phys. 8 (2020) 054 [arXiv:2001.05562] [INSPIRE]. 
[24] E. D'Hoker and D.H. Phong, The Box graph in superstring theory, Nucl. Phys. B 440 (1995) 24 [hep-th/9410152] [INSPIRE].

[25] M.B. Green and P. Vanhove, The low-energy expansion of the one loop type-II superstring amplitude, Phys. Rev. D 61 (2000) 104011 [hep-th/9910056] [INSPIRE].

[26] M.B. Green, J.G. Russo and P. Vanhove, Low energy expansion of the four-particle genus-one amplitude in type-II superstring theory, JHEP 02 (2008) 020 [arXiv:0801.0322] [INSPIRE].

[27] E. D'Hoker, M.B. Green and P. Vanhove, On the modular structure of the genus-one Type II superstring low energy expansion, JHEP 08 (2015) 041 [arXiv: 1502.06698] [INSPIRE].

[28] E. D'Hoker, M. Gutperle and D.H. Phong, Two-loop superstrings and S-duality, Nucl. Phys. B 722 (2005) 81 [hep-th/0503180] [INSPIRE].

[29] E. D'Hoker and M.B. Green, Zhang-Kawazumi Invariants and Superstring Amplitudes, arXiv: 1308.4597 [INSPIRE].

[30] E. D'Hoker, M.B. Green, B. Pioline and R. Russo, Matching the $D^{6} R^{4}$ interaction at two-loops, JHEP 01 (2015) 031 [arXiv:1405.6226] [INSPIRE].

[31] E. D'Hoker, M.B. Green and B. Pioline, Higher genus modular graph functions, string invariants, and their exact asymptotics, Commun. Math. Phys. 366 (2019) 927 [arXiv: 1712.06135] [INSPIRE].

[32] E. D'Hoker, M.B. Green and B. Pioline, Asymptotics of the $D^{8} \mathcal{R}^{4}$ genus-two string invariant, Commun. Num. Theor. Phys. 13 (2019) 351 [arXiv:1806.02691] [InSPIRE].

[33] E. D'Hoker, M.B. Green, O. Gürdogan and P. Vanhove, Modular Graph Functions, Commun. Num. Theor. Phys. 11 (2017) 165 [arXiv:1512.06779] [INSPIRE].

[34] O. Schlotterer and S. Stieberger, Motivic Multiple Zeta Values and Superstring Amplitudes, J. Phys. A 46 (2013) 475401 [arXiv:1205.1516] [InSPIRE].

[35] M.B. Green, C.R. Mafra and O. Schlotterer, Multiparticle one-loop amplitudes and S-duality in closed superstring theory, JHEP 10 (2013) 188 [arXiv:1307.3534] [INSPIRE].

[36] A. Basu, Simplifying the one loop five graviton amplitude in type IIB string theory, Int. J. Mod. Phys. A 32 (2017) 1750074 [arXiv: 1608.02056] [INSPIRE].

[37] M.R. Gaberdiel and M.B. Green, An $\mathrm{SL}(2, Z)$ anomaly in IIB supergravity and its F-theory interpretation, JHEP 11 (1998) 026 [hep-th/9810153] [INSPIRE].

[38] M.B. Green, Interconnections between type-II superstrings, $M$-theory and $N=4$ supersymmetric Yang-Mills, Lect. Notes Phys. 525 (1999) 22 [hep-th/9903124] [INSPIRE].

[39] R.H. Boels, Maximal R-symmetry violating amplitudes in type IIB superstring theory, Phys. Rev. Lett. 109 (2012) 081602 [arXiv: 1204.4208] [INSPIRE].

[40] M.B. Green and C. Wen, Modular Forms and $\mathrm{SL}(2, \mathbb{Z})$-covariance of type IIB superstring theory, JHEP 06 (2019) 087 [arXiv: 1904.13394] [INSPIRE].

[41] A. Basu, Eigenvalue equation for genus two modular graphs, JHEP 02 (2019) 046 [arXiv: 1812.00389] [INSPIRE].

[42] E. D'Hoker, M.B. Green and P. Vanhove, Proof of a modular relation between 1-, 2- and 3-loop Feynman diagrams on a torus, J. Number Theor. 196 (2019) 381 [arXiv:1509.00363] [INSPIRE]. 
[43] E. D'Hoker and M.B. Green, Identities between Modular Graph Forms, J. Number Theor. 189 (2018) 25 [arXiv: 1603.00839] [INSPIRE].

[44] E. D'Hoker and J. Kaidi, Hierarchy of Modular Graph Identities, JHEP 11 (2016) 051 [arXiv: 1608.04393] [INSPIRE].

[45] A. Basu, Proving relations between modular graph functions, Class. Quant. Grav. 33 (2016) 235011 [arXiv: 1606.07084] [INSPIRE].

[46] Z. Bern, L.J. Dixon, D.C. Dunbar, M. Perelstein and J.S. Rozowsky, On the relationship between Yang-Mills theory and gravity and its implication for ultraviolet divergences, Nucl. Phys. B 530 (1998) 401 [hep-th/9802162] [INSPIRE].

[47] D.M. Richards, The One-Loop Five-Graviton Amplitude and the Effective Action, JHEP 10 (2008) 042 [arXiv: 0807.2421] [INSPIRE].

[48] M.B. Green, J.G. Russo and P. Vanhove, String theory dualities and supergravity divergences, JHEP 06 (2010) 075 [arXiv: 1002.3805] [INSPIRE].

[49] B. Pioline, String theory integrands and supergravity divergences, JHEP 02 (2019) 148 [arXiv: 1810.11343] [INSPIRE].

[50] E. D'Hoker and D.H. Phong, Two-loop superstrings. V. Gauge slice independence of the N-point function, Nucl. Phys. B 715 (2005) 91 [hep-th/0501196] [INSPIRE].

[51] E. D'Hoker and D.H. Phong, Two loop superstrings. 1. Main formulas, Phys. Lett. B 529 (2002) 241 [hep-th/0110247] [INSPIRE].

[52] E. D'Hoker and D.H. Phong, Two loop superstrings. 2. The Chiral measure on moduli space, Nucl. Phys. B 636 (2002) 3 [hep-th/0110283] [inSPIRE].

[53] E. D'Hoker and D.H. Phong, Two loop superstrings. 3. Slice independence and absence of ambiguities, Nucl. Phys. B 636 (2002) 61 [hep-th/0111016] [INSPIRE].

[54] E. D'Hoker and D.H. Phong, Two loop superstrings 4: The Cosmological constant and modular forms, Nucl. Phys. B 639 (2002) 129 [hep-th/0111040] [INSPIRE].

[55] E. Witten, Notes On Holomorphic String And Superstring Theory Measures Of Low Genus, arXiv:1306.3621 [INSPIRE].

[56] N. Berkovits and C.R. Mafra, Equivalence of two-loop superstring amplitudes in the pure spinor and RNS formalisms, Phys. Rev. Lett. 96 (2006) 011602 [hep-th/0509234] [INSPIRE].

[57] H. Gomez and C.R. Mafra, The Overall Coefficient of the Two-loop Superstring Amplitude Using Pure Spinors, JHEP 05 (2010) 017 [arXiv: 1003.0678] [inSPIRE].

[58] N. Berkovits, Super Poincaré covariant quantization of the superstring, JHEP 04 (2000) 018 [hep-th/0001035] [INSPIRE].

[59] N. Berkovits, Explaining Pure Spinor Superspace, hep-th/0612021 [INSPIRE].

[60] C.R. Mafra and O. Schlotterer, Cohomology foundations of one-loop amplitudes in pure spinor superspace, arXiv:1408.3605 [INSPIRE].

[61] C.R. Mafra and O. Schlotterer, Multiparticle SYM equations of motion and pure spinor BRST blocks, JHEP 07 (2014) 153 [arXiv: 1404.4986] [INSPIRE].

[62] C.R. Mafra and O. Schlotterer, One-loop superstring six-point amplitudes and anomalies in pure spinor superspace, JHEP 04 (2016) 148 [arXiv: 1603.04790] [INSPIRE].

[63] C.R. Mafra and O. Schlotterer, http://www.southampton.ac.uk/ crm1n16/pss.html. 
[64] C.R. Mafra, PSS: A FORM Program to Evaluate Pure Spinor Superspace Expressions, arXiv: 1007.4999 [INSPIRE].

[65] C.L. Siegel, Topics in complex function theory. Vol. I,II,III, Wiley Interscience, Hoboken U.S.A. (1971).

[66] L. Álvarez-Gaumé, G.W. Moore and C. Vafa, Theta Functions, Modular Invariance and Strings, Commun. Math. Phys. 106 (1986) 1 [INSPIRE].

[67] B. Pioline, A Theta lift representation for the Kawazumi-Zhang and Faltings invariants of genus-two Riemann surfaces, J. Number Theor. 163 (2016) 520 [arXiv:1504.04182] [INSPIRE].

[68] J.E. Gerken, A. Kleinschmidt and O. Schlotterer, Heterotic-string amplitudes at one loop: modular graph forms and relations to open strings, JHEP 01 (2019) 052 [arXiv:1811.02548] [INSPIRE].

[69] J.E. Gerken, A. Kleinschmidt and O. Schlotterer, All-order differential equations for one-loop closed-string integrals and modular graph forms, JHEP 01 (2020) 064 [arXiv:1911.03476] [INSPIRE].

[70] J.E. Gerken, A. Kleinschmidt and O. Schlotterer, Generating series of all modular graph forms from iterated Eisenstein integrals, JHEP 07 (2020) 190 [arXiv:2004.05156] [INSPIRE].

[71] E. D'Hoker and O. Schlotterer, Identities among higher genus modular graph tensors, arXiv:2010.00924 [INSPIRE].

[72] A. Basu, Poisson equations for elliptic modular graph functions, arXiv:2009.02221 [INSPIRE].

[73] H. Gomez, One-loop Superstring Amplitude From Integrals on Pure Spinors Space, JHEP 12 (2009) 034 [arXiv:0910.3405] [INSPIRE].

[74] C.R. Mafra, Pure Spinor Superspace Identities for Massless Four-point Kinematic Factors, JHEP 04 (2008) 093 [arXiv:0801.0580] [INSPIRE].

[75] C. Vafa and E. Witten, A One loop test of string duality, Nucl. Phys. B 447 (1995) 261 [hep-th/9505053] [INSPIRE].

[76] Z. Bern, J.J.M. Carrasco and H. Johansson, New Relations for Gauge-Theory Amplitudes, Phys. Rev. D 78 (2008) 085011 [arXiv:0805.3993] [INSPIRE].

[77] N.E.J. Bjerrum-Bohr, P.H. Damgaard, T. Sondergaard and P. Vanhove, The Momentum Kernel of Gauge and Gravity Theories, JHEP 01 (2011) 001 [arXiv:1010.3933] [INSPIRE].

[78] C.R. Mafra, O. Schlotterer and S. Stieberger, Complete N-Point Superstring Disk Amplitude I. Pure Spinor Computation, Nucl. Phys. B 873 (2013) 419 [arXiv:1106.2645] [InSPIRE].

[79] F.A. Berends and W.T. Giele, Recursive Calculations for Processes with n Gluons, Nucl. Phys. B 306 (1988) 759 [INSPIRE].

[80] C.R. Mafra, O. Schlotterer, S. Stieberger and D. Tsimpis, A recursive method for SYM n-point tree amplitudes, Phys. Rev. D 83 (2011) 126012 [arXiv:1012.3981] [INSPIRE].

[81] C.R. Mafra and O. Schlotterer, Berends-Giele recursions and the BCJ duality in superspace and components, JHEP 03 (2016) 097 [arXiv: 1510.08846] [INSPIRE].

[82] R.H. Boels, On the field theory expansion of superstring five point amplitudes, Nucl. Phys. B 876 (2013) 215 [arXiv:1304.7918] [INSPIRE]. 
[83] J. Broedel, O. Schlotterer and S. Stieberger, https://wwwth.mpp.mpg.de/members/stieberg/mzv/index.html.

[84] H. Kawai, D.C. Lewellen and S.H.H. Tye, A Relation Between Tree Amplitudes of Closed and Open Strings, Nucl. Phys. B 269 (1986) 1 [INSPIRE].

[85] F. Zerbini, Single-valued multiple zeta values in genus 1 superstring amplitudes, Commun. Num. Theor. Phys. 10 (2016) 703 [arXiv:1512.05689] [INSPIRE].

[86] E. D'Hoker and M.B. Green, Absence of irreducible multiple zeta-values in melon modular graph functions, Commun. Num. Theor. Phys. 14 (2020) 315 [arXiv:1904.06603] [InSPIRE].

[87] D. Zagier and F. Zerbini, Genus-zero and genus-one string amplitudes and special multiple zeta values, Commun. Num. Theor. Phys. 14 (2020) 413 [arXiv:1906.12339] [InSPIRE].

[88] P. Vanhove and F. Zerbini, Building blocks of closed and open string amplitudes, in MathemAmplitudes 2019: Intersection Theory and Feynman Integrals, Padova Italy (2020) [arXiv: 2007.08981] [INSPIRE].

[89] M.B. Green, M. Gutperle and H.-h. Kwon, Sixteen fermion and related terms in M-theory on $T^{2}$, Phys. Lett. B 421 (1998) 149 [hep-th/9710151] [INSPIRE].

[90] J.D. Fay, Theta functions on Riemann surfaces, Lect. Notes Math. 352 (1973) 1.

[91] M. Schiffer and D.C. Spencer, Functionals of finite Riemann surfaces, Courier Corporation, North Chelmsford U.S.A. (2014).

[92] E. D'Hoker, A. Kleinschmidt and O. Schlotterer, Elliptic modular graph forms I: Identities and generating series, accepted for publication in JHEP, arXiv:2012.09198.

[93] J.E. Gerken and J. Kaidi, Holomorphic subgraph reduction of higher-point modular graph forms, JHEP 01 (2019) 131 [arXiv: 1809.05122] [INSPIRE].

[94] J.E. Gerken, Basis Decompositions and a Mathematica Package for Modular Graph Forms, arXiv: 2007.05476 [INSPIRE]. 